AperTO - Archivio Istituzionale Open Access dell'Università di Torino

\title{
Differential Harnack Inequalities and the Ricci Flow
}

\section{This is a pre print version of the following article:}

Original Citation:

\section{Availability:}

This version is available http://hdl.handle.net/2318/1701023

since 2020-01-08T14:28:57Z

Publisher:

Andrew Ranicki

Published version:

DOI:10.4171/030

Terms of use:

Open Access

Anyone can freely access the full text of works made available as "Open Access". Works made available under a Creative Commons license can be used according to the terms and conditions of said license. Use of all other works requires consent of the right holder (author or publisher) if not exempted from copyright protection by the applicable law. 
This is a preprint of the book published in

EMS Series Lect. Math. (2006)

DOI: $10.4171 / 030$

\title{
Differential Harnack Inequalities and the Ricci Flow
}

\author{
Reto Müller
}

Note: This is a preliminary version of my book. In particular, in this preprint the Preface, Table of Contents, List of Symbols, Index and all Pictures/Figures are missing. The book can be bought at EMS or AMS.

\section{Introduction}

In this book we compare the linear heat equation on a static manifold with the Ricci flow which is a nonlinear heat equation for a Riemannian metric $g(t)$ on $M$ - and with the heat equation on a manifold moving by Ricci flow. The aim of the book is to explain some of the theory of Perelman's first Ricci flow paper [28] as well as its thematic context. Concretely, we will discuss differential Harnack inequalities, entropy formulas and Perelman's $\mathcal{L}$-functional. In particular, we want to emphasize how these three topics are related with each other and with special solutions of the Ricci flow, so-called Ricci solitons. We will see that Li-Yau type Harnack inequalities are related with Perelman's entropy formulas and the $\mathcal{L}$-functional in a quite natural way. To motivate the results and to start with simpler computations, we will first consider the analogs of these topics for the heat equation on a static manifold. We will see that the results in the static and in the Ricci flow case are often very similar and sometimes coincide on special solutions.

This introduction contains a brief exposition of the historical background and summarizes the main content of the book.

\section{Hamilton's Ricci flow and its early success}

Nonlinear heat flows first appeared in Riemannian geometry in 1964, when James Eells and J.H. Sampson [10] introduced the harmonic map heat flow as a tool to deform given maps $u: M \rightarrow N$ between two manifolds into extremal maps (i.e. critical points in the sense of calculus of variations) for the energy functional

$$
E(u)=\int_{M}|\nabla u|^{2} d V .
$$

In particular, these so-called harmonic maps include geodesics, harmonic functions and minimal surfaces. Eells and Sampson showed that the gradient flow of the energy functional (0.1) above - the harmonic map heat flow - will converge to a harmonic map if the target manifold $N$ has negative sectional curvature. Since then, geometric heat flows have become an intensively studied topic in geometric analysis.

A fundamental problem in differential geometry is to find canonical metrics on Riemannian manifolds, i.e. metrics which are highly symmetrical, for example metrics with constant 
curvature in some sense. Using the idea of evolving an object to such an ideal state by a nonlinear heat flow, Richard Hamilton [13] invented the Ricci flow in 1981. Hamilton's idea was to smooth out irregularities of the curvature by evolving a given Riemannian metric on a manifold $M$ with respect to the nonlinear weakly parabolic equation

$$
\partial_{t} g_{i j}(t)=-2 R_{i j}(t),
$$

where $g_{i j}$ denotes the Riemannian metric and $R_{i j}$ its Ricci curvature. Hamilton showed that there exists a solution for a short time for any smooth initial metric $g_{0}$, see [13] (or also [9] for a simplified proof).

A solution metric $g(t)$ shrinks where its Ricci curvature is positive, while it expands in regions where the Ricci curvature is negative. For example a round sphere, which has positive constant Ricci curvature at time $t=0$, will shrink - faster and faster - and collapse to a single point in finite time. In particular, the volume of the sphere is strictly decreasing along the flow. Hamilton therefore also considered the normalized (i.e. volume preserving) version of the Ricci flow, given by the equation

$$
\partial_{t} g_{i j}=-2 R_{i j}+\frac{2}{n} r g_{i j},
$$

where $n$ denotes the dimension of $M$ and $r=\int_{M} R d V / \int_{M} d V$ is the average scalar curvature.

The stationary metrics under the Ricci flow (0.2) are Ricci flat metrics (i.e. metrics with Ric $\equiv 0)$. These are also the critical points of the Einstein-Hilbert functional

$$
\mathcal{E}(g)=\int_{M} R d V,
$$

a fact wich we will prove in chapter one. However, we will see that the gradient flow of this functional differs from the Ricci flow by an additional term, which makes the equation impossible to solve in general. More precisely, the gradient flow of (0.3) is given by the equation

$$
\partial_{t} g_{i j}=-2 R_{i j}+R g_{i j},
$$

which does not have to be solvable (in either time direction) even for a short time, since $\partial_{t} g=-2$ Ric is parabolic, while $\partial_{t} g=R g$ is backwards parabolic and at the symbol level these two terms do not cancel. One can hence regard the Ricci flow as the part of the Einstein-Hilbert gradient flow that one gets by cancelling out the bad (i.e. backwards parabolic) term. Now the natural question arises whether the Ricci flow is the gradient flow of any functional at all, but unfortunately the answer is no. This seems to be a well-known proposition of Hamilton, but we have not yet seen any published proof in the literature. A proof can be found in chapter one.

If the Riemannian metric evolves with respect to a nonlinear heat equation, then so do its derivatives, in particular the different curvature tensors. All these equations will be presented in chapter one. For example the scalar curvature satisfies

$$
\partial_{t} R=\triangle R+2|\mathrm{Ric}|^{2} \geq \triangle R+\frac{2}{n} R^{2},
$$

so by the maximum principle its minimum $R_{\min }(t)=\inf _{M} R(\cdot, t)$ is non-decreasing along the flow. Hamilton developed a maximum principle for tensors, with which he could find 
similar results for the Ricci and Riemannian curvature tensors. He proved that the Ricci flow preserves the positivity of the Ricci tensor in dimension three [13] and of the curvature operator in all dimensions [14]. Moreover, he also proved that the eigenvalues of the Ricci tensor in dimension three approach each other under the normalized flow. This allowed him to prove the following convergence result in his seminal paper.

Proposition 0.1 (Hamilton, [13])

For a three-manifold with initial metric of strictly positive Ricci tensor a solution to the normalized Ricci flow will exist for all time and will converge exponentially fast to a metric of constant positive sectional curvature as time t tends to infinity.

Such a three-manifold must hence be diffeomorphic to the three-sphere or a quotient of it by a finite group of isometries. Given a homotopy three-sphere, if one can show that it always admits a metric of positive Ricci curvature, then with Hamilton's result the Poincaré conjecture would follow. In [14], Hamilton proved a similar result for four-manifolds with initial metric of positive curvature operator.

However, such a simple result does not hold if one starts with an arbitrary metric without curvature assumptions. In the general case, the solution of the Ricci flow (0.2) may behave much more complicatedly and develop singularities in finite time, in particular the curvature may become arbitrarily large in some region while staying bounded in its complement. For example, if one starts with an almost round cylindrical neck, which looks like $S^{2} \times B^{1}$ connecting two large pieces of low curvature, then the positive curvature in the $S^{2}$-direction will dominate the slightly negative curvature in the $B^{1}$-direction and therefore one expects the neck to shrink and pinch off.

An existence proof and detailed analysis of such neckpinches can be found in a recent book by Bennett Chow and Dan Knopf [8], the first rigorous examples have been constructed by Sigurd Angenent and Dan Knopf in [1].

Remark. The above picture is justified by Angenent's and Knopf's paper [2], where they proved that the diameter of the neck remains finite and that the singularity occurs solely on a hypersurface diffeomorphic to $S^{2}$ rather than along $S^{2} \times[a, b]$, for instance.

In order to deal with such neckpinches, Hamilton [15] invented a topological surgery where one cuts the neck open and glues small caps to each of the boundaries in such a way that one can continue running the Ricci flow. He proposed a surgery procedure for four-manifolds that satisfy certain curvature assumptions and conjectured that a similar surgery would also work for three-manifolds with no a-priori assumptions at all. This led him to a program of attacking William Thurston's geometrization conjecture [29], which states that every closed three-manifold can be decomposed along spheres $S^{2}$ or tori $T^{2}$ into pieces that admit one of eight different geometric structures. In this context, neckpinch surgery corresponds to the topological decomposition along two-spheres into such pieces. However, neckpinches can also occur for purely PDE-related reasons, as in the picture above, where a sphere $S^{3}$ is decomposed into two spheres $S^{3}$. Note that attaching a three-sphere along a two-sphere to any manifold $M$ does not change the topology of $M$. A good source for Hamilton's program is his survey [19] from 1995. To analyze singularities, one can use an analytic tool that allows to compare the curvatures of the solution at different points and different times. This tool is known as a Harnack type inequality, which we will now describe. 


\section{Differential Harnack inequalities}

The classical Harnack inequality from parabolic PDE theory states that for $0<t_{1}<t_{2} \leq T$ a non-negative smooth solution $u \in C^{\infty}(M \times[0, T])$ of the linear heat equation $\partial_{t} u=\triangle u$ on a closed, connected manifold $M$ satisfies

$$
\sup _{M} u\left(\cdot, t_{1}\right) \leq C \inf _{M} u\left(\cdot, t_{2}\right),
$$

where $C$ depends on $t_{1}, t_{2}$ and the geometry of $M$. However, in the classical presentation, the geometric dependency of $C$ is not easy to analyze and the inequality does not provide the optimal comparison at points that are far from the infimum and the supremum.

In 1986, Peter Li and Shing Tung Yau [22] found a completely new Harnack type result, namely a pointwise gradient estimate (called a differential Harnack inequality) that can be integrated along a path to find a classical Harnack inequality of the form (0.6). They proved that on a manifold with Ric $\geq 0$ and convex boundary, the differential Harnack expression

$$
H(u, t):=\frac{\partial_{t} u}{u}-\frac{|\nabla u|^{2}}{u^{2}}+\frac{n}{2 t}
$$

is non-negative for any positive solution $u$ of the linear heat equation. To prove this differential Harnack inequality one uses the maximum principle for parabolic equations. With his maximum principle for systems mentioned above, Hamilton [17] was able to prove a matrix version of the Li-Yau result. On the one hand Hamilton's result is more general since his matrix inequality contains the Li-Yau inequality as its trace, on the other hand the matrix inequality demands stronger curvature assumptions than Li-Yau's trace version. We will see in chapter two that the Li-Yau Harnack expression $H(u, t)$ as well as Hamilton's matrix version vanish identically on the heat kernel (which is the expanding self-similar solution of the heat equation which tends to a $\delta$-function as $t \rightarrow 0$ ).

For the Ricci flow, the self-similar solutions are called Ricci solitons. Concretely, a Ricci soliton is a solution metric of the Ricci flow which moves only by diffeomorphisms and scaling, i.e. a solution $g(t)=a(t) \cdot \Phi_{t}^{*}(g(0))$ which is the pull-back of the initial metric $g(0)$ by a one-parameter family of diffeomorphisms $\Phi_{t}: M \rightarrow M$ multiplied with a scaling function $a(t)$. An easy example for a shrinking (i.e. $a(t)$ decreasing) soliton is the sphere $S^{n}$ discussed above. If the scaling function $a(t)$ equals 1 for all time, then the soliton is called steady.

The simplest nontrivial example here is the so-called cigar soliton, which is defined to be $\mathbb{R}^{2}$ equipped with the metric

$$
d s^{2}=\frac{d x^{2}+d y^{2}}{1+x^{2}+y^{2}} .
$$

It is called a cigar because it is asymptotic to a cylinder at infinity, has maximal Gauss curvature at the origin and burns away.

If the diffeomorphisms in the definition of a soliton are generated by the gradient of some scalar function $f$ on $M$ - the so-called soliton potential - we call $g(t)$ a gradient soliton.

Hamilton's idea was to find a nonlinear analogon to the Li-Yau Harnack inequality involving positive curvature in place of $u$. Motivated by the fact that Li Yau's Harnack expression and his own matrix version are zero on expanding self-similar solutions as mentioned above, he looked for curvature expressions which vanish on expanding Ricci solitons and then searched for a linear combination of these expressions and derivatives of it. With this idea he found a matrix and a trace Harnack inequality for the Ricci flow, cf. [18]. We will present them at 
the end of chapter two.

Using his Harnack inequalities, Hamilton [19] was able to prove the following classification of blow-ups of three-dimensional singularities - modulo the control of the injectivity radius.

Proposition 0.2 (Hamilton, theorem 26.5 of [19])

Let $(M, g(t))$ be a solution to the Ricci flow on a compact three-manifold where a singularity develops in finite time $T$. Then either the injectivity radius times the square root of the maximum curvature goes to zero, or else there exists a sequence of dilations of the solution which converges to a quotient by isometries of either $S^{3}, S^{2} \times \mathbb{R}$ or $\Sigma \times \mathbb{R}$, where $\Sigma$ is the cigar soliton.

Notice that all three possible limits in the proposition are gradient solitons, the first two being shrinking and the last one being steady. A key question for Hamilton's program is: How can one prove that all singularities are modelled by self-similar solutions? This question was answered by Grisha Perelman [28], who introduced a new differential Harnack inequality for the Ricci flow, described below. In fact, Hamilton's and Perelman's Harnack estimates and gradient Ricci solitons are important for the study of the flow in arbitrary dimensions, not only in dimension three.

\section{Perelman's gradient flow approach}

Recall that it was Hamilton's idea to take linear combinations of expressions that vanish on soliton solutions in order to find interesting estimates. Therefore, we list various vanishing expressions of a soliton potential $f$ for steady, shrinking and expanding gradient solitons in chapter one. Combining some of them, one finds the remarkable result that in the steady soliton case the function $e^{-f}$ satisfies the adjoint heat equation on a manifold evolving by Ricci flow. Similar results hold for expanding and shrinking solitons. This connection between Ricci solitons and the adjoint heat equation is the starting point for a completely new approach to the subject, introduced by Perelman [28] in 2002.

Perelman presented a new functional, which may be regarded as an improved version of the Einstein-Hilbert functional (0.3), namely

$$
\mathcal{F}(g, f):=\int_{M}\left(R+|\nabla f|^{2}\right) e^{-f} d V
$$

which has the gradient flow system

$$
\begin{cases}\partial_{t} g_{i j} & =-2\left(R_{i j}+\nabla_{i} \nabla_{j} f\right) \\ \partial_{t} f & =-\Delta f-R\end{cases}
$$

After a pull-back by the family of diffeomorphisms generated by $\nabla f$, this system becomes

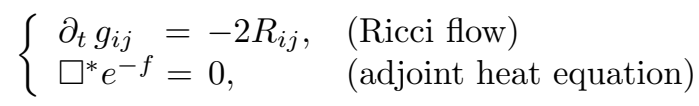

where $\square^{*}:=-\partial_{t}-\triangle+R$ denotes the adjoint heat operator under the Ricci flow. So in this sense the Ricci flow may be regarded as a gradient flow up to a modification with a family of diffeomorphisms. Note that if we interpret the Ricci flow as a dynamical system on 
the space of Riemannian metrics modulo diffeomorphisms and scaling, the soliton solutions correspond to fixed points in this space.

In chapter three we will show that the entropy functional $\mathcal{F}(g, f)$ is non-decreasing along the flow and constant exactly on steady Ricci solitons with potential $f$. Similar entropy functionals to (0.9) have been found for shrinking solitons (by Perelman [28]) and later for expanding solitons (by Feldman, Ilmanen and Lei Ni [12]) as well. We will introduce them all in the third chapter. We will also explain the corresponding entropy functionals for the heat equation on a static manifold. This is taken from Lei Ni's papers [25] and [26].

In Perelman's paper [28] no clear motivation for the various entropy functionals can be found, so at first read it is almost impossible to understand where these functionals come from, even if one already knows the connection between solitons and the adjoint heat equation mentioned above. But using Lei Ni's result (for the heat equation on a fixed manifold), we will see that the entropy functionals are quite natural and are strongly connected to the Nash entropy and, surprisingly, also to the Li-Yau Harnack inequality. This relationship will be our main interest in chapter three.

In this chapter, we also show how one finds another differential Harnack inequality for the heat kernel via a local version of Ni's entropy formula (cf. [25]). In the Ricci flow case, this corresponds to Perelman's Harnack inequality for the adjoint heat kernel on a manifold evolving by Ricci flow (cf. [28], section 9), which one gets via a local version of the shrinking soliton entropy. Note that while the classical Li-Yau result presented in chapter two holds for the linear heat equation on a static manifold and Hamilton's Harnack inequalities are results for the curvature under Ricci flow (i.e. a nonlinear heat equation on the space of Riemannian metrics), Perelman's Harnack inequality involves both heat equations together: it holds for a solution of the (adjoint) heat equation on a manifold that also evolves by a heat equation.

In the same way as one can integrate the Li-Yau Harnack estimate along a path to get a classical Harnack inequality, one can also integrate Perelman's Harnack inequality and the new Harnack inequality that corresponds to Lei Ni's entropy formula. One gets a lower bound for the adjoint heat kernel under Ricci flow in the Perelman case or for the heat kernel on a static manifold in the other case. This will lead us to Perelman's $\mathcal{L}$-length functional (cf. [28], section 7), a functional defined on space-time paths. We will discuss this functional, the corresponding space-time geodesics and exponential map in chapter four. We will also introduce and analyze the corresponding "length" functional for the static case. The new results that we will get there follow as a natural continuation of Lei Ni's results. On flat $\mathbb{R}^{n}$ the two cases coincide modulo the necessary sign changes.

One finds an interesting fact there: If one computes the first and second variation of Perelman's $\mathcal{L}$-functional with respect to variations of the space-time path - remember that we found this functional by integrating Perelman's Harnack inequality along a path - one finds Hamilton's matrix and trace Harnack expressions for the Ricci flow. So these two (at first glance completely different) Harnack expressions come together in this chapter on the $\mathcal{L}$ functional in a very natural way. Surprisingly, to draw conclusions, Hamilton's Harnack inequalities for the Ricci flow do not have to be satisfied, since the Harnack terms appear during the computations but cancel out in the final result! If one does the analogous computation for the static case, the Ricci curvature appears where Hamilton's Harnack expressions 
appeared in the Ricci flow case. However, the curvature terms do not cancel out and hence the conclusions one wants to make on the corresponding $\mathcal{L}$-length (as well as the monotonicity of Ni's entropy functionals mentioned above or the positivity of Li-Yau's Harnack expression) only hold if the Ricci curvature is non-negative. This shows that the results for the Ricci flow case - even so they are harder to compute - are in fact more natural since they hold without any a-priori curvature assumptions converse to the static case.

Here is a heuristic explanation why the results for the linear heat equation hold exactly in the case of Ric $\geq 0$ : In this case the constant metric $\tilde{g}(t) \equiv g(0)$ is a supersolution to the solution of the Ricci flow $\partial_{t} g=-2$ Ric $\leq 0$. Moreover a positive solution to the backwards heat equation $\partial_{t} u=-\triangle u$ is a subsolution to the adjoint heat equation under the Ricci flow $\partial_{t} u=-\Delta u+R u$ (since $R \geq 0$ if Ric $\geq 0$ ). Putting this together, the inequalities which hold for the Ricci flow and the adjoint heat equation will also hold for the backwards heat equation on a manifold with fixed metric. Changing the time direction will then lead to the results for the (forward) heat equation. With this explanation in mind, there are two ways of looking at this monograph. One can either start with the static case and interpret the results in the Ricci flow case as more complicated analogs. This is how historically the Harnack inequalities have been found and it also gives an easier approach to Perelman's entropy formulas and $\mathcal{L}$-geodesics, even so the presentation is then chronologically reversed in chapter three and four. Since the computations in the case of a fixed metric are usually much easier, we have chosen this order to present the results. Alternatively one could also see the Ricci flow combined with the adjoint heat equation as the geometrically natural case and - in contrast to our point of presentation - interpret the formulas for the static case as an application of the corresponding formulas for the Ricci flow to a super- respectively subsolution in the special case of non-negative Ricci curvature.

For this book, we have condensed some of the already existing proofs or carried them out in more detail to make the topic more accessible to non-experts. However, it is always a good idea to look at the original sources, especially since some propositions are not presented in their most general version here, which would only distract from the main geometric ideas sometimes. In particular, we recommend Hamilton's original sources (especially [13], [17] and [18]) and Lei Ni's papers [25] and [26] on Perelman's ideas.

To not get sidetracked too much, we only present a compact core of the subject without too many applications. In particular, we will not discuss any of the applications for threemanifolds towards the Poincaré conjecture or Thurston's geometrization conjecture at all, but only present results which are valid for arbitrary dimensions. But there are also various applications for general dimensions (such as non-collapsing, no breathers, $\varepsilon$-regularity, etc.) which we skip. Most of them can be found in Perelman's paper [28].

Let me stress again that I do not claim any original work. The only new results (in chapter three and four) are natural simplifications of Perelman's work applied to Lei Ni's entropy formula for the static case.

\section{Outline of the book}

Let me now explain the outline of the book in more detail and with references to the most important results.

The book is divided into four main chapters. In chapter one we explain general varia- 
tions of Riemannian metrics and introduce the Ricci flow as the (weakly) parabolic part of the $L^{2}$-gradient flow of the Einstein-Hilbert functional. We then prove that there exists no functional which has the Ricci flow as its gradient flow (proposition 1.7). After computing various evolution equations for the Ricci flow, we introduce gradient Ricci solitons and derive equations for the three cases of steady, shrinking and expanding solitons (propositions 1.14, 1.15 and 1.16). A reader who is familiar with Hamilton's papers will already know most of the results in this chapter.

Chapter two is devoted to the study of differential Harnack inequalities. We start with the famous Li-Yau Harnack estimate, which we present in its original form (proposition 2.5) as well as in a quadratic and an integrated version (corollaries 2.6 and 2.7). We then explain the maximum principle for systems to prove Hamilton's matrix Harnack inequality for the heat equation (proposition 2.11). Finally, we proceed with Hamilton's Harnack inequalities for the Ricci flow (theorem 2.14), where we only give a heuristic motivation instead of rewriting the rather lengthy proof.

In the third chapter we present Lei Ni's entropy formulas for the heat equation on a static manifold. The main result (theorem 3.8) will relate these entropy formulas with the Nash entropy and the Li-Yau Harnack inequality. Moreover, a local version of an entropy formula for the positive heat kernel (proposition 3.6) will lead us to a new Li-Yau type differential Harnack inequality, which gives a lower bound for the heat kernel when being integrated (corollary 3.10). We also discuss the entropy formulas for steady, shrinking and expanding solitons, the most important being the shrinking case in which we will again integrate a Harnack type inequality (proposition 3.15) to find a lower bound for the adjoint heat kernel under Ricci flow (corollary 3.16), as in section 9 of Perelman's paper [28]. The quantities $\ell(q, \bar{\tau})$ and $\ell(q, T)$ which one finds in these two corollaries 3.16 and 3.10, respectively, will turn out to be Perelman's backwards reduced distance and its analog for the heat kernel on a static manifold.

This motivates our investigation of the two corresponding distance-functionals in chapter four. We first examine the static case, where the computations are much easier. This will lead to proposition 4.2, a new result for the heat kernel on a manifold, which coincides with Perelman's result (theorem 4.9) in the case of a Ricci flat manifold. The second part of chapter four provides a detailed exposition of Perelman's $\mathcal{L}$-length, $\mathcal{L}$-geodesics and the $\mathcal{L}$ exponential map. We finish with the monotonicity of Perelman's backwards reduced volume (corollary 4.13).

\section{Development of the book and acknowledgements}

In the summer semester 2004, Tom Ilmanen held an introductory lecture on the Ricci flow at ETH Zürich that was inspired by Perelman's paper from 2002 and Ilmanen's discussions with Richard Hamilton, Mu-Tao Wang and Lei Ni. Invited guest speakers involved Richard Hamilton, Dan Knopf and Natasa Sesum. This lecture was the reason I became interested in the subject of geometric flows, entropy formulas and Harnack inequalities and I would like to thank Tom Ilmanen as well as the above mentioned guest speakers very much for it. Much of the first chapter as well as some parts of the third chapter are inspired by this lecture.

This book was written as my diploma thesis in the winter semester 2004/2005 at ETH Zürich under the guidance of Michael Struwe. I wish to express my gratitude to Michael 
Struwe for many interesting and helpful discussions, comments and suggestions during this semester. I would also like to thank Daniel Perez for proof-reading parts of my diploma thesis.

In the winter semester 2005/2006, I organized a student seminar on geometric heat flows together with Tom Ilmanen, where my thesis was used as a source which was often easier to understand for the students than the original papers. So in the present (slightly revised) form, we think that this book can be used for an introductory lecture or seminar on this topic, but it is also adapted for self-study - even for undergraduate students or people who are new to this subject.

\section{Foundational material}

In this preliminary chapter we briefly explain the objects and notations which we will use throughout this book. The reader is expected to be familiar with the most basic theory from Riemannian geometry, in particular with the notion of Riemannian manifolds, connections, geodesics and (curvature) tensors. For the convenience of the reader we first summarize without proofs some of the relevant material, thus making our exposition as self-contained as possible. For the proofs we refer the reader to [21]. Afterwards, we introduce the Ricci flow and explain some of its most important properties. Especially the evolution equations derived here will be of great importance for the following chapters. We will see that the evolution equations for Riemannian, Ricci and scalar curvature all have the form of heat equations, too. We then deduce the adjoint heat equation under Ricci flow. Finally, we define Ricci flow solitons and show how gradient solitons and adjoint heat equations are connected. For a more detailed introduction on the Hamilton-Ricci flow, we refer to Chow's and Knopf's book [8], Topping's lecture notes [30] or Hamilton's survey [13].

\subsection{Riemannian metric and curvature tensors}

Let $M$ be a closed Riemannian manifold. Our notation is similar to the standard classical notation for Riemannian geometry as found in [21]. We write $v^{i}$ for vectors and $v_{j}$ for co-vectors, meaning $v^{i} \frac{\partial}{\partial x^{i}}$ and $v_{j} d x^{j}$, respectively. We write $\partial_{k}$ as an abbreviation for $\frac{\partial}{\partial x^{k}}$ and $\nabla_{k}$ for the covariant derivative in the direction of $\frac{\partial}{\partial x^{k}}$.

The Riemannian metric on $M$ is denoted as $g_{i j}=g_{j i}$, its inverse as $g^{k \ell}$, so that $g_{i j} g^{j k}=\delta_{i}^{k}$. For the induced measure on $M$ we write $d V=\sqrt{\operatorname{det}\left(g_{i j}\right)} d x$. We always use the extended Einstein summation convention, where $x^{a} y_{a}$ means $\sum_{a=1}^{n} x^{a} y_{a}$, and $x_{a} y_{a}$ denotes $g^{a b} x_{a} y_{b}=\sum_{a, b=1}^{n} g^{a b} x_{a} y_{b}$.

We will mix this classical index notation with the nice coordinate-free notation, where usually the underlying geometric idea can better be seen, because the index notation is well-adapted to the intense computations we will perform in this book.

The Levi-Cività connection $\nabla_{i} v^{k}=\partial_{i} v^{k}+\Gamma_{i j}^{k} v^{j}$ is determined by the Christoffel symbols

$$
\Gamma_{i j}^{k}:=\frac{1}{2} g^{k \ell}\left(\partial_{i} g_{j \ell}+\partial_{j} g_{i \ell}-\partial_{\ell} g_{i j}\right),
$$

and the Riemannian curvature tensor

$$
\operatorname{Rm}(X, Y) Z=\nabla_{X} \nabla_{Y} Z-\nabla_{Y} \nabla_{X} Z-\nabla_{[X, Y]} Z=R_{\ell i j}^{k} X^{i} Y^{j} Z^{\ell}
$$


has the local representation

$$
R_{\ell i j}^{k}=\partial_{i} \Gamma_{\ell j}^{k}-\partial_{\ell} \Gamma_{i j}^{k}+\Gamma_{\ell j}^{m} \Gamma_{i m}^{k}-\Gamma_{i j}^{m} \Gamma_{\ell m}^{k} .
$$

If we lower the upper index to the first position, we get $R_{k \ell i j}=g_{k h} R_{\ell i j}^{h}$, a tensor which is anti-symmetric in $(k, \ell)$ and $(i, j)$, and symmetric in the interchange of these pairs, $R_{i j k \ell}=$ $R_{k \ell i j}$. The Ricci tensor Ric is defined by $R_{i k}=g^{j \ell} R_{i j k \ell}$, the scalar curvature as its trace $R=g^{i k} R_{i k}=g^{i k} g^{j \ell} R_{i j k \ell}$. It is easy to see that

$$
R_{k i}=R_{i k}=g^{h \ell} R_{h i \ell k}=g^{h \ell} g_{h j} R_{i \ell k}^{j}=R_{i j k}^{j} .
$$

We write $\langle X, Y\rangle:=g(X, Y)=g_{i j} X^{i} Y^{j}$ for the induced inner product of the metric $g$. If $\left\{e_{1}, \ldots, e_{n}\right\}$ is an orthonormal basis with respect to this inner product, then

$$
\operatorname{Ric}(X, Y)=\sum_{i=1}^{n}\left\langle\operatorname{Rm}\left(X, e_{i}\right) e_{i}, Y\right\rangle, \quad \text { and } \quad R=\sum_{i=1}^{n} \operatorname{Ric}\left(e_{i}, e_{i}\right) .
$$

For $p \in M$, one defines the exponential map $\exp _{p}: V_{p} \rightarrow M, v \mapsto \gamma_{v}(1)$, where $\gamma_{v}(t)$ : $[0, T] \rightarrow M$ is the unique maximal geodesic with $\gamma_{v}(0)=p$ and $\partial_{t} \gamma_{v}(0)=v$, and $V_{p}:=$ $\left\{v \in T_{p} M \mid \gamma_{v}\right.$ is defined on $\left.[0,1]\right\}$, cf. [21], definition 1.4.3. The exponential map $\exp _{p}$ maps a neighborhood $V$ of $0 \in T_{p} M$ diffeomorphically onto a neighborhood $U$ of $p \in M$ (see theorem 1.4.3 of [21]). So by identification of $T_{p} M$ with $\mathbb{R}^{n}$, the map $x(q)=\exp _{p}^{-1}(q)$ defines a local chart of $U$ with $p$ mapped to $x(p)=0$. The local coordinates defined by this chart $(x, U)$ are called (Riemannian) normal coordinates.

Lemma 1.1 (metric in normal coordinates)

In Riemannian normal coordinates $x=\left\{x^{i}\right\}$ around $x(p)=0$, we have

$$
g_{i j}(x)=\delta_{i j}-\frac{1}{3} R_{i p j q} x^{p} x^{q}+O\left(|x|^{3}\right),
$$

so in particular

$$
g_{i j}(0)=\delta_{i j}, \partial_{k} g_{i j}(0)=0, \Gamma_{i j}^{k}(0)=0,
$$

and

$$
\triangle^{\text {coord. }}\left(g_{i j}\right):=\left(\sum_{k=1}^{n} \frac{\partial^{2}}{\partial\left(x^{k}\right)^{2}} g_{i j}\right)=-\frac{2}{3} R_{i j} .
$$

Proof. It is a standard fact that $g_{i j}(0)=\delta_{i j}$ and $\partial_{k} g_{i j}(0)=0$ (and hence also $\left.\Gamma_{i j}^{k}(0)=0\right)$ for normal coordinates, cf. [21], theorem 1.4.4. We can then compute

$$
\begin{aligned}
2 R_{i p j q} & =2 g_{i k} R_{p j q}^{k}=2 g_{i k}\left(\partial_{j} \Gamma_{p q}^{k}-\partial_{p} \Gamma_{j q}^{k}\right) \\
& =g_{i k} g^{k \ell}\left(\partial_{j} \partial_{q} g_{p \ell}+\partial_{j} \partial_{p} g_{q \ell}-\partial_{j} \partial_{\ell} g_{p q}-\partial_{p} \partial_{q} g_{j \ell}-\partial_{p} \partial_{j} g_{q \ell}+\partial_{p} \partial_{\ell} g_{j q}\right) \\
& =\partial_{j} \partial_{q} g_{p i}-\partial_{j} \partial_{i} g_{p q}-\partial_{p} \partial_{q} g_{i j}+\partial_{p} \partial_{i} g_{j p} .
\end{aligned}
$$

In normal coordinates, the metric satisfies the symmetries

$$
\begin{gathered}
\partial_{i} \partial_{j} g_{p q}=\partial_{p} \partial_{q} g_{i j}, \\
\partial_{a} \partial_{b} g_{c d}+\partial_{b} \partial_{c} g_{a d}+\partial_{c} \partial_{a} g_{b d}=0,
\end{gathered}
$$

so (1.3) simplifies to

$$
R_{i p j q}=\partial_{j} \partial_{q} g_{p i}-\partial_{p} \partial_{q} g_{i j}
$$


By Taylor approximation, we have

$$
g_{i j}(x)=\delta_{i j}+\frac{1}{2} \partial_{p} \partial_{q} g_{i j} x^{p} x^{q}+O\left(|x|^{3}\right),
$$

and hence it only remains to show that

$$
\frac{1}{2} \partial_{p} \partial_{q} g_{i j} x^{p} x^{q}=-\frac{1}{3} R_{i p j q} x^{p} x^{q} .
$$

We compute

$$
\begin{aligned}
-\frac{1}{3} R_{i p j q} x^{p} x^{q} & =-\frac{1}{6}\left(R_{i p j q}+R_{i q j p}\right) x^{p} x^{q} \\
& =-\frac{1}{6}\left(\partial_{j} \partial_{q} g_{i p}+\partial_{p} \partial_{j} g_{i q}-2 \partial_{p} \partial_{q} g_{i j}\right) x^{p} x^{q} \\
& =-\frac{1}{6}\left(-3 \partial_{p} \partial_{q} g_{i j}\right) x^{p} x^{q}=\frac{1}{2} \partial_{p} \partial_{q} g_{i j} x^{p} x^{q},
\end{aligned}
$$

where we used (1.6) for the second line and the symmetry (1.5) for the last line. The claim follows.

In the following, we also need the Bianchi identities for the Riemannian curvature tensor, i.e. the first Bianchi identity

$$
\operatorname{Rm}(X, Y) Z+\operatorname{Rm}(Y, Z) X+\operatorname{Rm}(Z, X) Y=0,
$$

and the second Bianchi identity

$$
\left\langle\left(\nabla_{X} \operatorname{Rm}\right)(Y, Z) V, W\right\rangle+\left\langle\left(\nabla_{Y} \operatorname{Rm}\right)(Z, X) V, W\right\rangle+\left\langle\left(\nabla_{Z} \operatorname{Rm}\right)(X, Y) V, W\right\rangle=0,
$$

which implies the local formula at $x(p)=0$

$$
\nabla_{h} R_{i j k \ell}+\nabla_{k} R_{i j \ell h}+\nabla_{\ell} R_{i j h k}=0 .
$$

Tracing this with $g^{i h}$ gives the formula

$$
\begin{aligned}
\nabla_{i} R_{i j k \ell} & =g^{i h} \nabla_{h} R_{i j k \ell}=-g^{i h} \nabla_{k} R_{i j \ell h}-g^{i h} \nabla_{\ell} R_{i j h k} \\
& =\nabla_{k} R_{j \ell}-\nabla_{\ell} R_{j k} .
\end{aligned}
$$

This formula can be used to change derivatives of the Riemannian curvature into derivatives of the Ricci curvature. By tracing again with $g^{j \ell}$ we get another form of this equation, which will allow us to change derivatives of the Ricci tensor into derivatives of the scalar curvature. We find

$$
\nabla_{i} R_{i k}=g^{j \ell} \nabla_{i} R_{i j k \ell}=g^{j \ell} \nabla_{k} R_{j \ell}-g^{j \ell} \nabla_{\ell} R_{j k}=\nabla_{k} R-\nabla_{j} R_{j k} .
$$

By changing the indices in the last term, this implies

$$
\nabla_{i} R_{i k}=\frac{1}{2} \nabla_{k} R
$$




\subsection{Variation formulas}

We vary a fixed metric $g_{i j} \in \Gamma\left(\operatorname{Sym}_{+}^{2}\left(T^{*} M\right)\right)$ in direction $h_{i j} \in \Gamma\left(\operatorname{Sym}^{2}\left(T^{*} M\right)\right)$ to obtain $g^{s}=g+s h$. For any functional $\mathcal{F}(g)$ depending on $g$, we denote by $\delta_{h} \mathcal{F}$ or by $\partial_{s} \mathcal{F}$ the first variation

$$
\delta_{h} \mathcal{F}=\partial_{s} \mathcal{F}:=\left.\partial_{s}\right|_{s=0} \mathcal{F}\left(g_{i j}+s h_{i j}\right) .
$$

The variation of other objects depending on the Riemannian metric is understood in an analogous sense.

Definition 1.2 (Lichnerowicz Laplacian)

For a symmetric two-tensor $t_{i k}$ we define the Lichnerowicz Laplacian $\triangle_{L}$ by the formula

$$
\triangle_{L} t_{i k}:=\triangle t_{i k}+2 R_{i p k q} t_{p q}-R_{i p} t_{p k}-R_{k p} t_{p i}
$$

where $\triangle t_{i k}:=g^{j \ell} \nabla_{j} \nabla_{\ell} t_{i k}$ is the raw Laplacian.

Remark. The Lichnerowicz Laplacian for a symmetric two-tensor is formally the same as the Hodge-de Rham Laplacian for a two-form, see [8], appendix A.4.

Definition 1.3 (divergence operator)

For a one-form $\eta=\left(\eta_{i}\right)$ and a symmetric two-tensor $t=\left(t_{i k}\right)$ we define the divergence as $\operatorname{div} \eta:=\nabla_{i} \eta_{i}$ and $(\operatorname{div} t)_{k}:=\nabla_{i} t_{i k}$, respectively. In the second case, we then find the adjoint operator $\operatorname{div}^{*}: \Omega^{1}(M) \rightarrow \Gamma\left(\operatorname{Sym}^{2}\left(T^{*} M\right)\right)$ of $\operatorname{div}: \Gamma\left(\operatorname{Sym}^{2}\left(T^{*} M\right)\right) \rightarrow \Omega^{1}(M)$ to be

$$
\operatorname{div}^{*} \eta=-\frac{1}{2}\left(\nabla_{i} \eta_{k}+\nabla_{k} \eta_{i}\right)
$$

Proposition 1.4 (variation of curvature)

Let $\partial_{s} g_{i j}=h_{i j}$ be a variation of the Riemannian metric. In normal coordinates at $x(p)=0$, we then get

$$
\begin{aligned}
\partial_{s} R_{i j k}^{\ell}= & \frac{1}{2} g^{\ell m}\left(\nabla_{j} \nabla_{k} h_{i m}-\nabla_{j} \nabla_{m} h_{i k}-\nabla_{i} \nabla_{k} h_{j m}+\nabla_{i} \nabla_{m} h_{j k}\right. \\
& \left.-R_{i j k p} h_{p m}-R_{i j m p} h_{k p}\right) \\
\partial_{s} R_{i k}= & -\frac{1}{2} \triangle_{L} h_{i k}-\frac{1}{2} \nabla^{2}\left(\operatorname{tr}_{g} h\right)-\operatorname{div}^{*} \operatorname{div} h \\
\partial_{s} R= & -h^{i k} R_{i k}-\triangle\left(\operatorname{tr}_{g} h\right)+\operatorname{div}^{2} h
\end{aligned}
$$

The above general variation formulas can be used to compute evolution equations for the curvature under different flows, such as Einstein flow, Yamabe flow or - as we will do a few pages below - for the Ricci flow.

Proof. With $0=\partial_{s} \delta_{\ell}^{i}=\partial_{s}\left(g^{i k} g_{k \ell}\right)=\left(\partial_{s} g^{i k}\right) g_{k \ell}+g^{i k} h_{k \ell}$ we find

$$
\partial_{s} g^{i j}=\left(\partial_{s} g^{i k}\right) \delta_{k}^{j}=g^{j \ell}\left(\partial_{s} g^{i k}\right) g_{k \ell}=-g^{j \ell} g^{i k} h_{k \ell}=:-h^{i j} .
$$

If we choose normal coordinates for $g$, the definition of the Christoffel symbols implies

$$
\partial_{s} \Gamma_{i k}^{\ell}=\frac{1}{2} g^{\ell m}\left(\nabla_{i} h_{k m}+\nabla_{k} h_{i m}-\nabla_{m} h_{i k}\right)
$$


at $x=0$. Since the Christoffel symbols vanish in normal coordinates, the Riemannian curvature tensor is given by $R_{i j k}^{\ell}=\partial_{j} \Gamma_{i k}^{\ell}-\partial_{i} \Gamma_{j k}^{\ell}$. Thus

$$
\begin{aligned}
\partial_{s} R_{i j k}^{\ell}= & \frac{1}{2} g^{\ell m}\left(\nabla_{j} \nabla_{k} h_{i m}-\nabla_{j} \nabla_{m} h_{i k}-\nabla_{i} \nabla_{k} h_{j m}+\nabla_{i} \nabla_{m} h_{j k}\right. \\
& \left.+\nabla_{j} \nabla_{i} h_{k m}-\nabla_{i} \nabla_{j} h_{k m}\right),
\end{aligned}
$$

which implies (1.9), because

$$
\nabla_{j} \nabla_{i} h_{k m}-\nabla_{i} \nabla_{j} h_{k m}=-R_{i j k p} h_{p m}-R_{i j m p} h_{k p} .
$$

To compute (1.10) we use $R_{i k}=R_{i j k}^{j}$, which yields

$$
\begin{aligned}
\partial_{s} R_{i k}= & \frac{1}{2}\left(\nabla_{m} \nabla_{k} h_{i m}-\triangle h_{i k}-\nabla_{i} \nabla_{k} g^{j m} h_{j m}+\nabla_{i} \nabla_{j} h_{j k}\right. \\
& \left.-R_{i j k p} h_{p j}+R_{i p} h_{k p}\right) \\
= & \frac{1}{2}\left(\nabla_{k} \nabla_{m} h_{i m}-\triangle h_{i k}-2 R_{i j k p} h_{p j}+R_{k p} h_{p i}+R_{i p} h_{k p}\right. \\
& \left.-\nabla_{i} \nabla_{k}\left(\operatorname{tr}_{g} h\right)+\nabla_{i} \nabla_{j} h_{j k}\right) \\
= & \frac{1}{2}\left(-\triangle_{L} h_{i k}-\nabla_{i} \nabla_{k}\left(\operatorname{tr}_{g} h\right)+\nabla_{k}(\operatorname{div} h)_{i}+\nabla_{i}(\operatorname{div} h)_{k}\right) \\
= & -\frac{1}{2} \triangle_{L} h_{i k}-\frac{1}{2} \nabla^{2}\left(\operatorname{tr}_{g} h\right)-\operatorname{div}^{*} \operatorname{div} h .
\end{aligned}
$$

Finally, we have

$$
\begin{aligned}
\partial_{s} R= & \partial_{s}\left(g^{i k} R_{i k}\right)=-h^{i k} R_{i k}+g^{i k}\left(\partial_{s} R_{i k}\right) \\
= & -h^{i k} R_{i k}+\frac{1}{2} g^{i k}\left(-\triangle h_{i k}-\nabla_{i} \nabla_{k}\left(\operatorname{tr}_{g} h\right)+\nabla_{k}(\operatorname{div} h)_{i}+\nabla_{i}(\operatorname{div} h)_{k}\right. \\
& \left.-2 R_{i j k p} h_{p j}+R_{k p} h_{p i}+R_{i p} h_{k p}\right) \\
= & -h^{i k} R_{i k}+\frac{1}{2}\left(-\triangle\left(\operatorname{tr}_{g} h\right)-\triangle\left(\operatorname{tr}_{g} h\right)+\nabla_{i}(\operatorname{div} h)_{i}+\nabla_{k}(\operatorname{div} h)_{k}\right. \\
& \left.-2 R_{j p} h_{p j}+R_{i p} h_{p i}+R_{k p} h_{k p}\right) \\
= & -h^{i k} R_{i k}-\triangle\left(\operatorname{tr}_{g} h\right)+\operatorname{div}^{2} h,
\end{aligned}
$$

which proves the last formula.

Proposition 1.5 (variation of $d V$ )

Let $\partial_{s} g_{i j}=h_{i j}$, then the volume element satisfies

$$
\partial_{s} d V=\left(\frac{1}{2} t r_{g} h\right) d V .
$$

Proof. W use the following classical result from linear algebra. Let $A=\left(a_{i j}\right)$ and $B=\left(b_{i j}\right)$ be two matrices and let $A: B$ denote the product $a_{i j} b_{i j}$. If $\operatorname{det}(A) \neq 0$, then $\left.\partial_{s}\right|_{s=0} \operatorname{det}(A+$ $s B)=\left(A^{-1}: B\right) \operatorname{det}(A)$. In our case

$$
\partial_{s} \operatorname{det}(g)=g^{i j} h_{i j} \operatorname{det}(g)=:\left(\operatorname{tr}_{g} h\right) \operatorname{det}(g) .
$$

Hence, we find the variation of $d V$ to be

$$
\partial_{s} d V=\partial_{s} \sqrt{\operatorname{det}(g)} d x=\frac{\partial_{s} \operatorname{det}(g)}{2 \sqrt{\operatorname{det}(g)}} d x=\left(\frac{1}{2} t r_{g} h\right) \sqrt{\operatorname{det}(g)} d x=\left(\frac{1}{2} t r_{g} h\right) d V .
$$




\subsection{Einstein-Hilbert functional and Ricci flow}

The Einstein-Hilbert functional is defined as

$$
\mathcal{E}(g)=\int_{M} R d V
$$

With the results from the previous section, we can now easily compute its first variation. With proposition 1.4 and 1.5 we find

$$
\begin{aligned}
\partial_{s} \mathcal{E} & =\int_{M}\left(\partial_{s} R d V+R \partial_{s} d V\right) \\
& =\int_{M}\left[\left(-h^{i k} R_{i k}-\triangle t r_{g} h+\operatorname{div}^{2} h\right)+\left(R \frac{1}{2} t r_{g} h\right)\right] d V \\
& =\int_{M} h^{i k}\left(-R_{i k}+\frac{1}{2} R g_{i k}\right) d V \\
& =\int_{M}-h^{i k} E_{i k} d V=\int_{M}\langle h, \nabla \mathcal{E}\rangle d V,
\end{aligned}
$$

where $E_{i k}=R_{i k}-\frac{1}{2} R g_{i k}$ is the Einstein tensor. We thus get $\partial_{t} g=\nabla \mathcal{E}=-E$ as the gradient flow of $\mathcal{E}$. For the third line, note that on a closed manifold $M$ the integral of the divergence form $\left(-\triangle t r_{g} h+\operatorname{div}^{2} h\right)$ disappears.

Definition 1.6 (stationary metrics)

We call $g$ stationary for $\mathcal{E}$, if $\delta_{h} \mathcal{E}=0$ for all $h \in \Gamma\left(\operatorname{Sym}^{2}\left(T^{*} M\right)\right)$. Since $E_{i k}=E_{k i}$, this is the case if and only if $E_{i k} \equiv 0$ on $M$. Taking the trace we get $0 \equiv g^{i k} R_{i k}-\frac{1}{2} R g^{i k} g_{i k}=\frac{2-n}{2} R$, so in dimension $n \neq 2$ this implies $R \equiv 0$ on $M$ and therefore Ric $\equiv 0$ on $M$. One calls such a manifold Ricci flat. In dimension $n=2$, every Riemannian metric satisfies $E \equiv 0$.

For the gradient flow of the Einstein-Hilbert functional we have (modified by multiplication with factor 2)

$$
\partial_{t} g_{i j}=-2 E_{i j}=-2 R_{i j}+R g_{i j}
$$

which one can prove is not a parabolic flow and which does not have to be solvable even for a short time. So we only take the (weakly) parabolic part of the equation, which leads to Hamilton's famous Ricci flow equation

$$
\partial_{t} g=-2 \text { Ric }
$$

By comparing this with (1.2), we see that in Riemannian normal coordinates this equation - up to a multiplicative constant - is simply the heat equation $\partial_{t} g_{i j}=3 \triangle^{\text {coord. }}\left(g_{i j}\right)$ for the Riemannian metric.

Remark. Alternatively, one can chose harmonic coordinates $\left\{x^{i}\right\}$, i.e. local coordinates for which each coordinate function $x^{i}$ satisfies

$$
0=\triangle x^{i}=g^{j k}\left(\partial_{j} \partial_{k}-\Gamma_{j k}^{\ell} \partial_{\ell}\right) x^{i} .
$$

In such harmonic coordinate, the Ricci flow takes the form

$$
\partial_{t} g_{i j}=\triangle^{\text {coord }} \cdot\left(g_{i j}\right)+Q_{i j}\left(g^{-1}, \partial g\right),
$$

where $Q$ denotes a sum of terms which are quadratic in the inverse $g^{-1}$ and first derivatives $\partial g$ of the metric $g$, see [8], chapter 3.5. 
For every smooth metric $g_{i j}=g_{i j}(0)$ the Ricci flow equation has a solution $g_{i j}(t)$ for at least a short time $t \in[0, \varepsilon)$, see [13] for Hamilton's original proof using the Nash-Moser implicit function theorem, or see [9] for a simplified short-time existence proof using the fact that (1.14) is equivalent to a strictly parabolic system modulo the action of the diffeomorphism group on $M$. On the other side, the Ricci flow is not a gradient flow:

Proposition 1.7 (Hamilton)

There exists no smooth functional

$$
\mathcal{F}(g)=\int F\left(\partial^{2} g, \partial g, g\right) d V
$$

such that its first variation is $\partial_{s} \mathcal{F}=\int\langle h,-2$ Ric $\rangle d V$.

Proof. The idea of the following proof was proposed to the author by Tom Ilmanen. Fix a metric $g \in \Gamma\left(\operatorname{Sym}_{+}^{2}\left(T^{*} M\right)\right)$ and let $h$ and $k$ be symmetric two-tensors $h, k \in \Gamma\left(\operatorname{Sym}^{2}\left(T^{*} M\right)\right)$ with which we vary $g$. Since $C^{\infty}\left(\Gamma\left(\operatorname{Sym}_{+}^{2}\left(T^{*} M\right)\right)\right)$ is a linear space, it has a natural connection $\nabla$ satisfying

$$
\nabla \mathcal{F}_{g}(h):=\left.\partial_{t}\right|_{t=0} \mathcal{F}(g+t h) .
$$

We compute

$$
\nabla^{2} \mathcal{F}_{g}(h, k)=\left.\partial_{s} \partial_{t}\right|_{(s, t)=(0,0)} \mathcal{F}(g+t h+s k)=\left.\partial_{s}\right|_{s=0} \nabla \mathcal{F}_{g+s k}(h),
$$

and hence the symmetry of $\nabla^{2} \mathcal{F}_{g}(h, k)$ requires that the map

$$
T:\left.(h, k) \mapsto \partial_{s}\right|_{s=0} \nabla \mathcal{F}_{g+s k}(h)
$$

should be symmetric in $(h, k)$. We now assume that there exists indeed a functional $\mathcal{F} \in$ $C^{\infty}\left(\Gamma\left(\operatorname{Sym}_{+}^{2}\left(T^{*} M\right)\right)\right)$ that has the Ricci flow as its gradient flow, i.e.

$$
\nabla \mathcal{F}_{g}(h)=\int_{M}\langle h,-2 \text { Ric }\rangle d V .
$$

With the notation $g^{s}=g+s k$, we compute

$$
\begin{aligned}
& T(h, k)=\left.\partial_{s}\right|_{s=0} \int_{M}\left\langle h,-2 \operatorname{Ric}\left(g^{s}\right)\right\rangle_{g^{s}} d V_{g^{s}} \\
&=-\left.2 \int_{M}\left(\left\langle h, \partial_{s} \operatorname{Ric}\left(g^{s}\right)\right\rangle d V+\partial_{s}\langle h, \operatorname{Ric}\rangle_{g^{s}} d V+\langle h, \operatorname{Ric}\rangle \partial_{s} d V_{g^{s}}\right)\right|_{s=0} \\
&=-2 \int_{M}\left(\left\langle h,-\frac{1}{2} \triangle_{L} k-\frac{1}{2} \nabla^{2}\left(\operatorname{tr}_{g} k\right)-\operatorname{div}^{*} \operatorname{div} k\right\rangle\right. \\
&\left.\quad-2 \operatorname{tr}_{g}(k: \operatorname{Ric}: h)+\langle h, \operatorname{Ric}\rangle\left(\frac{1}{2} \operatorname{tr}_{g} k\right)\right) d V \\
& \sim-\int_{M}\left(\nabla\left(\operatorname{tr}_{g} k\right) \cdot \operatorname{div} h+\langle h, \operatorname{Ric}\rangle \operatorname{tr}_{g} k\right) d V
\end{aligned}
$$

where we used $\sim$ to indicate that we dropped all terms symmetric in $h$ and $k$ in the last step. Now (1.15) is manifestly not symmetric - unless $h$ and $k$ satisfy some special differential identities - and we get the desired contradiction.

Perelman [28] solved this problem by introducing a functional, whose gradient flow is equivalent to the Ricci flow modified with a family of diffeomorphisms. We will see this functional in chapter three. 


\subsection{Evolution equations under Ricci flow}

When the Riemannian metric evolves, then so does its curvature. For the Ricci flow, one can now derive evolution equations from the general variation formulas in proposition 1.4 by setting $h:=-2$ Ric. Define $B_{i j k \ell}:=R_{i p j q} R_{k p \ell q}$, the square of Rm.

Theorem 1.8 (evolution of curvature)

Let $g(t)$ be a solution of the Ricci flow equation (1.14). Then

$$
\begin{aligned}
\partial_{t} R_{i j k \ell}= & \triangle R_{i j k \ell}+2\left(B_{i j k \ell}-B_{i j \ell k}+B_{i k j \ell}-B_{i \ell j k}\right) \\
& -R_{i p} R_{p j k \ell}-R_{j p} R_{i p k \ell}-R_{k p} R_{i j p \ell}-R_{\ell p} R_{i j k p}, \\
\partial_{t} R_{i k}= & \triangle_{L} R_{i k}=\triangle R_{i k}+2 R_{p q} R_{i p k q}-2 R_{i p} R_{p k}, \\
\partial_{t} R= & \triangle R+2|\mathrm{Ric}|^{2} .
\end{aligned}
$$

Proof. We won't prove (1.16), because we will not need it in the following chapters. For a direct proof of this equation without the variation formula from proposition 1.4, see [13]. To prove (1.17), note that with the twice contracted second Bianchi identity (1.8) one gets

$$
\begin{aligned}
-\frac{1}{2} \nabla^{2}\left(\operatorname{tr}_{g} h\right)-\operatorname{div}^{*} \operatorname{div} h & =\nabla^{2}\left(\operatorname{tr}_{g} \operatorname{Ric}\right)-\left(\nabla_{k} \nabla_{j} R_{i j}+\nabla_{i} \nabla_{j} R_{j k}\right) \\
& =\nabla^{2} R-\left(\frac{1}{2} \nabla_{k} \nabla_{i} R+\frac{1}{2} \nabla_{i} \nabla_{k} R\right) \\
& =\nabla^{2} R-\nabla^{2} R=0,
\end{aligned}
$$

and hence proposition 1.4 implies

$$
\partial_{t} R_{i k}=\triangle_{L} R_{i k}=\triangle R_{i k}+2 R_{p q} R_{i p k q}-2 R_{i p} R_{p k} .
$$

For the scalar curvature formula, use proposition 1.4, equation (1.11), to get

$$
\begin{aligned}
\partial_{t} R & =-h^{i k} R_{i k}-\triangle\left(\operatorname{tr}_{g} h\right)+\operatorname{div}^{2} h \\
& =2|\operatorname{Ric}|^{2}+2 \triangle R-2 \nabla_{i} \nabla_{j} R_{i j} .
\end{aligned}
$$

With (1.8) we get $-2 \nabla_{i} \nabla_{j} R_{i j}=-\triangle R$, and equation (1.18) follows.

Notice that the evolution equation (1.12) for the Christoffel symbols gives the evolution for the Levi-Cività connection $\nabla_{i} v^{k}=\partial_{i} v^{k}+\Gamma_{i j}^{k} v^{j}$. However, we want to derive the evolution of the connection in a more elegant and coordinate-free form. This index-free equation will then be used at various places in chapter four. We need the following formula.

\section{Lemma 1.9}

The Levi-Cività connection satisfies the equation

$$
\begin{aligned}
2\left\langle\nabla_{X} Y, Z\right\rangle= & X\langle Y, Z\rangle-Z\langle X, Y\rangle+Y\langle X, Z\rangle \\
& -\langle[Y, Z], X\rangle+\langle[X, Y], Z\rangle-\langle[X, Z], Y\rangle .
\end{aligned}
$$

Proof. Since $\nabla$ is metric, i.e. $\nabla g=0$, it satisfies

$$
\begin{aligned}
& X\langle Y, Z\rangle=\left\langle\nabla_{X} Y, Z\right\rangle+\left\langle Y, \nabla_{X} Z\right\rangle, \\
& Y\langle Z, X\rangle=\left\langle\nabla_{Y} Z, X\right\rangle+\left\langle Z, \nabla_{Y} X\right\rangle, \\
& Z\langle X, Y\rangle=\left\langle\nabla_{Z} X, Y\right\rangle+\left\langle X, \nabla_{Z} Y\right\rangle .
\end{aligned}
$$


Since $\nabla$ is also torsion free, i.e. $\nabla_{X} Y-\nabla_{Y} X=[X, Y]$ for all $X, Y \in \Gamma(T M)$, we conclude

$$
\begin{aligned}
X\langle Y, Z\rangle-Y\langle Z, X\rangle+Z\langle X, Y\rangle= & 2\left\langle\nabla_{X} Y, Z\right\rangle-\langle[X, Y], Z\rangle \\
& +\langle Y,[X, Z]\rangle+\langle X,[Y, Z]\rangle,
\end{aligned}
$$

which is obviously equivalent to the assertion of the lemma.

Remark. In fact, the Levi-Cività connection is uniquely determined by the formula above, see theorem 3.3.1 of [21].

We now define $\dot{\nabla}=\partial_{t} \nabla$ by $\dot{\nabla}_{X} Y:=\partial_{t}\left(\nabla_{X} Y\right)$ for (time-independent) vector fields $X, Y \in$ $\Gamma(T M)$ and a time-dependent metric $g(t)$ on $M$. Note that since the difference of two connections $\nabla^{(1)}$ and $\nabla^{(2)}$ on $M$ is a tensor, $\dot{\nabla}$ is also a tensor - in contrary to the connection $\nabla$ itself. We now write $h=\partial_{t} g$ and differentiate the equation from the lemma above. This yields

$$
\begin{aligned}
2\left\langle\dot{\nabla}_{X} Y, Z\right\rangle+2 h\left(\nabla_{X} Y, Z\right)= & X \cdot h(Y, Z)-Z \cdot h(X, Y)+Y \cdot h(X, Z) \\
& -h([Y, Z], X)+h([X, Y], Z)-h([X, Z], Y) .
\end{aligned}
$$

Hence, we get

$$
\begin{aligned}
2\left\langle\dot{\nabla}_{X} Y, Z\right\rangle= & \left(\nabla_{X} h\right)(Y, Z)-h\left(\nabla_{X} Y, Z\right)+h\left(\nabla_{X} Z, Y\right)-\left(\nabla_{Z} h\right)(X, Y) \\
& -h\left(\nabla_{Z} X, Y\right)-h\left(\nabla_{Z} Y, X\right)+\left(\nabla_{Y} h\right)(X, Z)+h\left(\nabla_{Y} X, Z\right) \\
& +h\left(\nabla_{Y} Z, X\right)-h([Y, Z], X)+h([X, Y], Z)-h([X, Z], Y) \\
= & \left(\nabla_{X} h\right)(Y, Z)-\left(\nabla_{Z} h\right)(X, Y)+\left(\nabla_{Y} h\right)(X, Z),
\end{aligned}
$$

where the last equality follows directly from the fact that the Lie-brackets satisfy $[X, Y]=$ $\nabla_{X} Y-\nabla_{Y} X$ for the Levi-Cività connection. Setting $h=-2$ Ric, we have thus proved

Proposition 1.10 (evolution of $\nabla$ )

Let $g(t)$ be a solution of the Ricci flow equation (1.14). Then the Levi-Cività connection satisfies

$$
\left\langle\dot{\nabla}_{X} Y, Z\right\rangle=-\left(\nabla_{X} \operatorname{Ric}\right)(Y, Z)+\left(\nabla_{Z} \operatorname{Ric}\right)(X, Y)-\left(\nabla_{Y} \operatorname{Ric}\right)(X, Z) .
$$

With proposition 1.5 we find the evolution equation for the volume element:

$$
\partial_{t} d V=\left(\frac{1}{2} \operatorname{tr}_{g} h\right) d V=\frac{1}{2} g^{i j}\left(-2 R_{i j}\right) d V=-R d V
$$

This equation will not only be used to compute variations of integral functionals, but also for partial integrations with respect to the time $t$. As an application, we can now derive the evolution equation for the Laplace operator.

We define the operator $\dot{\Delta}=\partial_{t} \triangle$ by $\dot{\triangle} f:=\partial_{t}(\triangle f)$ for a (time-independent) function $f \in C^{\infty}(M)$ and a time-dependent metric $g(t)$ on $M$. We will use this operator in chapter three. We get the following evolution equation.

Proposition 1.11 (evolution of $\triangle$ )

Let $g(t)$ solve the Ricci flow equation. Then the Laplace operator on $C^{\infty}(M)$ satisfies

$$
\dot{\triangle}=2 R_{i j} \nabla_{i} \nabla_{j} .
$$


Proof. Let $f, h \in C^{\infty}(M)$ be arbitrary functions. With partial integration (and using $\partial M=$ $\emptyset)$ we find

$$
\int_{M} h \triangle f d V=-\int_{M}\langle\nabla h, \nabla f\rangle d V=-\int_{M} g^{i j} \nabla_{i} h \nabla_{j} f d V .
$$

If we differentiate both sides with respect to $t$, we get

$$
\int_{M}\left[h \dot{\triangle} f d V+h \triangle f\left(\partial_{t} d V\right)\right]=-\int_{M}\left[\left(\partial_{t} g^{i j}\right) \nabla_{i} h \nabla_{j} f d V+g^{i j} \nabla_{i} h \nabla_{j} f\left(\partial_{t} d V\right)\right] .
$$

Using $\partial_{t} d V=-R d V$ and $\partial_{t} g^{i j}=2 R^{i j}$, we obtain

$$
\begin{aligned}
\int_{M}[\dot{\triangle} f-R \triangle f] h d V & =-\int_{M}\left[2 R_{i j} \nabla_{j} f-R g^{i j} \nabla_{j} f\right]\left(\nabla_{i} h\right) d V \\
& =+\int_{M} \nabla_{i}\left[2 R_{i j} \nabla_{j} f-R g^{i j} \nabla_{j} f\right] h d V .
\end{aligned}
$$

This holds for all $h \in C^{\infty}(M)$, hence

$$
\begin{aligned}
\dot{\triangle} f-R \triangle f & =\nabla_{i}\left[2 R_{i j} \nabla_{j} f-R g^{i j} \nabla_{j} f\right] \\
& =\left(2 \nabla_{i} R_{i j}\right) \nabla_{j} f+2 R_{i j} \nabla_{i} \nabla_{j} f-g^{i j} \nabla_{i} R \nabla_{j} f-R g^{i j} \nabla_{i} \nabla_{j} f \\
& =\nabla_{j} R \nabla_{j} f+2 R_{i j} \nabla_{i} \nabla_{j} f-\nabla_{j} R \nabla_{j} f-R \triangle f \\
& =2 R_{i j} \nabla_{i} \nabla_{j} f-R \triangle f,
\end{aligned}
$$

which proves $\dot{\triangle} f=2 R_{i j} \nabla_{i} \nabla_{j} f, \forall f \in C^{\infty}(M)$.

Remark. Of course, one could also derive this formula by differentiating $\triangle f:=g^{i j} \nabla_{i} \nabla_{j} f$ and using the evolution equations for $g^{-1}$ and $\nabla$, which we already know.

Sometimes, one likes to consider the backwards Ricci flow equation $\partial_{\tau} g(\tau)=2$ Ric, where $g(t)$ is a solution of the forward Ricci flow for $t \in[-T, 0]$ (after a time shift), and $\tau$ is defined as $\tau:=-t$. Note that we can adapt all the evolution equations above for the backwards Ricci flow, by simply replacing $\partial_{t}$ derivatives with $\left(-\partial_{\tau}\right)$ derivatives.

\subsection{Adjoint heat equation and gradient solitons}

As mentioned in the introduction, we now want to introduce gradient Ricci solitons and derive various equations for them. In particular, we want to show the connection between their potential function and solutions of the adjoint heat equation.

We define the heat operator $\square:=\partial_{t}-\triangle$ on $M_{T}=M \times[0, T]$. Some may know this symbol as the wave operator, but throughout this book it will always denote the heat operator in accordance to the notation of Perelman's paper [28]. The heat equation can hence be written as $\square u=\partial_{t} u-\Delta u=0$. For $u$ and $v$ which satisfy the boundary condition $\int_{M} u(T) v(T) d V=\int_{M} u(0) v(0) d V$, we claim that the formal adjoint heat operator under Ricci flow is $\square^{*}:=-\partial_{t}-\triangle+R$, i.e. the adjoint heat equation is $\square^{*} v=0$. To see this, we use $\partial_{t} d V=-R d V$ and integration by parts. We get

$$
\begin{aligned}
\int_{M_{T}}(\square u) v d V d t & =\int_{M_{T}}\left(\partial_{t} u\right) v d V d t-\int_{M_{T}}(\triangle u) v d V d t \\
& =-\int_{M_{T}} u\left(\partial_{t} v d V+v \partial_{t} d V\right) d t-\int_{M_{T}} u(\triangle v) d V d t \\
& =\int_{M_{T}} u\left(-\partial_{t} v-\triangle v+R v\right) d V d t=\int_{M_{T}} u\left(\square^{*} v\right) d V d t
\end{aligned}
$$


which proves the claim. We will also need the following.

\section{Lemma 1.12}

Let $f, h \in C^{\infty}(M \times[0, T))$ be two arbitrary functions, $\square$ and $\square^{*}$ the heat operator and its formal adjoint, defined as above. Then

$$
\left.\partial_{t} \int_{M} f h d V=\int_{M}(\square f) h-f\left(\square^{*} h\right)\right) d V
$$

Proof. We have

$$
\partial_{t} \int_{M} f h d V=\int_{M}\left(\partial_{t} f\right) h d V+\int_{M} f\left(\partial_{t} h\right) d V+\int_{M} f h\left(\partial_{t} d V\right) .
$$

With integration by parts, we obtain

$$
0=\int_{M}(f \triangle h-h \triangle f) d V .
$$

Adding the two equations gives

$$
\begin{aligned}
\partial_{t} \int_{M} f h d V & \left.=\int_{M}\left(\partial_{t} f-\triangle f\right) h-f\left(\partial_{t} h-\triangle h+R\right)\right) d V \\
& =\int_{M}\left((\square f) h-f\left(\square^{*} h\right)\right) d V,
\end{aligned}
$$

which is what we claimed.

A solution to an evolution equation which changes under a one-parameter subgroup of the symmetry group of the equation is called a soliton (or a self-similar solution). The symmetry group of the Ricci flow contains all diffeomorphisms. We hence consider metrics which move by a one-parameter group of diffeomorphisms (and scaling), i.e. we consider solutions of the Ricci flow, which are fixed points modulo diffeomorphism and scaling:

Definition 1.13 (Ricci solitons)

We call a solution $g(t)$ of (1.14) a Ricci soliton, if it is the pull-back of $g(0)$,

$$
g(t)=a(t) \Phi_{t}^{*}(g(0))
$$

where $\left(\Phi_{t}\right)$ is a family of diffeomorphisms: $M \rightarrow M$ with $\Phi_{0}=\mathrm{id}_{M}$, and a $(t)$ is a real-valued function. The cases $\frac{d a}{d t}=a^{\prime}<0, a^{\prime} \equiv 0$ and $a^{\prime}>0$ correspond to shrinking, steady and expanding Ricci solitons, respectively. We will often simply call them shrinkers, steadies and expanders.

Now assume $\Phi_{t}$ is generated by a vector field $X$, i.e. $\partial_{t} \Phi_{t}(p)=X \circ \Phi_{t}(p)$. We call $g(t)=$ $\Phi_{t}^{*}(g(0))$ a gradient steady soliton, if $X$ is the gradient of a function $f$, i.e. $X_{i}=\nabla_{i} f$, $X^{i}=g^{i j} \nabla_{j} f$.

Proposition 1.14 (equations for gradient steadies)

Suppose $g(t)=\Phi_{t}^{*}(g(0))$ is a gradient steady soliton with potential $f$. Then we have

i) $\operatorname{Ric}+\operatorname{Hess}(f)=0$, 
ii) $R+\triangle f=0$

iii) $|\nabla f|^{2}+R \equiv$ const,

iv) $\partial_{t} f=|\nabla f|^{2}$.

Proof. If we differentiate $g(t)=\Phi_{t}^{*}(g(0))$ with respect to $t$, use (1.14) and the definition of the Lie-derivative $L_{X} g$ (cf. [21], definition 1.6.6), we get

$$
-2 R_{i j}=\partial_{t} g_{i j}=\left(L_{X} g\right)_{i j}=-2 \operatorname{div}^{*} X=\nabla_{i} X_{j}+\nabla_{j} X_{i},
$$

and hence $-2 R_{i j}=2 \nabla_{i} \nabla_{j} f$, which proves the first equation. The computation for $L_{X} h$, where $h$ is a two-tensor, is explained in [21], after theorem 1.6.4. The second equation is just the trace of the first one. To derive the third equation, we take $\nabla$ of i), permute the indices and subtract the two equations:

$$
\begin{aligned}
& \nabla_{k} R_{i j}+\nabla_{k} \nabla_{i} \nabla_{j} f=0 \\
& \nabla_{i} R_{k j}+\nabla_{i} \nabla_{k} \nabla_{j} f=0 \\
\Rightarrow & \nabla_{k} R_{i j}-\nabla_{i} R_{k j}+R_{k i j p} \nabla_{p} f=0 .
\end{aligned}
$$

Now we trace this equation with $g^{k j}$ and get

$$
\nabla_{j} R_{i j}-\nabla_{i} R+R_{i p} \nabla_{p} f=0 .
$$

Note that the twice contracted second Bianchi identity (1.8) and i) imply $\nabla_{j} R_{i j}=\frac{1}{2} \nabla_{i} R$ and $R_{i p}=-\nabla_{i} \nabla_{p} f$, and hence

$$
0=-\frac{1}{2} \nabla_{i} R-\nabla_{i} \nabla_{p} f \nabla_{p} f=-\frac{1}{2} \nabla_{i}\left(R+|\nabla f|^{2}\right),
$$

which proves iii). Finally, by definition

$$
f(t, p)=f\left(0, \Phi_{t}(p)\right), \quad \text { i.e. } \quad f(t, \cdot)=\Phi_{t}^{*}(f(0, \cdot)) .
$$

So differentiating with respect to $t$ proves iv), namely

$$
\partial_{t} f=L_{X} f=X \cdot \nabla f=|\nabla f|^{2} .
$$

As stated in the introduction, it was Hamilton's idea to take linear combinations of these equations for solitons. Here, by combining equation ii) with iv), $\partial_{t} f=|\nabla f|^{2}=|\nabla f|^{2}-$ $(R+\triangle f)$, one obtains

$$
\left(\partial_{t}+\triangle\right) f=|\nabla f|^{2}-R .
$$

So if one defines $u:=e^{-f}$, one finds

$$
\left(\partial_{t}+\triangle\right) u=\left(-\partial_{t} f-\triangle f+|\nabla f|^{2}\right) u=R u,
$$

i.e. $u=e^{-f}$ solves the adjoint heat equation

$$
\square^{*} u=-\partial_{t} u-\triangle u+R u=0 .
$$


Remark. It is usually not possible to solve such an equation in positive time direction, because it is backwards parabolic. Normally one therefore has to solve the Ricci flow equation (forward in time) first and then go back in time solving this adjoint heat equation. More precisely, one takes a solution $g(t)$ of the Ricci flow equation for $t \in[0, T]$ and fixes some final time Cauchy data $u(T)=u_{0}$. One can then solve the parabolic equation

$$
\partial_{\tau} u(\tau)-\triangle u(\tau)+R u(\tau)=0,
$$

with initial data $u(\tau=0)=u_{0}$. With $\tau=T-t$, this leads to a solution $u(t)$ of the backwards parabolic equation.

Similar to the steady case, a shrinking soliton is called gradient shrinker if the generating vector field $X(p):=-\partial_{t} \Phi_{t}(p)$ has a potential $f$. We assume that $a(t)=(T-t)$, thus $g(t)=(T-t) \Phi_{t}^{*}(g(0))$. This involves no loss of generality. We will often write $\tau$ instead of $(T-t)$ in the shrinking case.

Proposition 1.15 (equations for gradient shrinkers)

Let $g(t)$ be a gradient shrinker with potential $f, g(t)=(T-t) \Phi_{t}^{*}(g(0))$. Then $f$ satisfies the equations

i) $\operatorname{Ric}+\operatorname{Hess}(f)-\frac{g}{2 \tau}=0$,

ii) $R+\triangle f-\frac{n}{2 \tau}=0$,

iii) $|\nabla f|^{2}+R-\frac{f}{\tau} \equiv$ const,

iv) $\partial_{t} f=|\nabla f|^{2}=-\partial_{\tau} f$.

Proof. Differentiating $g(t)=(T-t) \Phi_{t}^{*}(g(0))$ and using (1.14) implies

$$
-2 R_{i j}=-\frac{g_{i j}}{\tau}+\left(L_{X} g\right)_{i j}=-\frac{g_{i j}}{\tau}+\nabla_{i} X_{j}+\nabla_{j} X_{i},
$$

hence $-2 R_{i j}=2 \nabla_{i} \nabla_{j} f-\frac{g_{i j}}{\tau}$, which proves i). The second equation is again the trace of the first one. Because $\nabla g=0$, we can continue by taking $\nabla$ of i) as in the steady case to get the same equation, namely

$$
\nabla_{j} R_{i j}-\nabla_{i} R+R_{i p} \nabla_{p} f=0
$$

where now $R_{i p}=-\nabla_{i} \nabla_{p} f+\frac{g_{i p}}{2 \tau}$. Hence we find

$$
0=-\frac{1}{2} \nabla_{i} R-\nabla_{i} \nabla_{p} f \nabla_{p} f-\frac{g_{i p}}{2 \tau} \nabla_{p} f=-\frac{1}{2} \nabla_{i}\left(R+|\nabla f|^{2}+\frac{f}{\tau}\right) .
$$

This proves the third equation. The last one follows as in proposition 1.14.

We find again a connection to the adjoint heat equation: Combining the second and last equations, one gets

$$
\partial_{t} f=|\nabla f|^{2}-\left(R+\triangle f-\frac{n}{2 \tau}\right),
$$

that is,

$$
\left(\partial_{t}+\triangle\right) f=|\nabla f|^{2}-R+\frac{n}{2 \tau},
$$


analogous to (1.23). We now define $u:=(4 \pi \tau)^{-n / 2} e^{-f}$ and compute

$$
\left(\partial_{t}+\triangle\right) u=\left(\frac{n}{2 \tau}-\partial_{t} f-\triangle f+|\nabla f|^{2}\right) u=R u,
$$

so the function $u=(4 \pi \tau)^{-n / 2} e^{-f}$ solves the adjoint heat equation

$$
\square^{*} u=-\partial_{t} u-\triangle u+R u=0 .
$$

The expanding case is pretty much the same as the shrinking case, there are only some sign changes. For $t>T$, let $g(t)$ be a gradient expander, i.e. a solution of the form $g(t)=(t-T) \Phi_{t}^{*}(g(0))$, where the generating $X(p):=\partial_{t} \Phi_{t}(p)$ has a potential. We often write $\sigma$ for $(t-T)$ in the expanding case.

If we replace $\tau$ by $-\sigma$ and $\partial_{\tau}$ by $\left(-\partial_{t}\right)$ in the proof of the above proposition, we immediately get

Proposition 1.16 (equations for gradient expanders)

The soliton potential $f$ of a gradient expander satisfies the following equations

i) $\operatorname{Ric}+\operatorname{Hess}(f)+\frac{g}{2 \sigma}=0$,

ii) $R+\triangle f+\frac{n}{2 \sigma}=0$,

iii) $|\nabla f|^{2}+R+\frac{f}{\sigma} \equiv$ const,

iv) $\partial_{t} f=|\nabla f|^{2}$.

Similarly to (1.24) we find

$$
\left(\partial_{t}+\triangle\right) f=|\nabla f|^{2}-R-\frac{n}{2 \sigma} .
$$

Now define $u:=(4 \pi \sigma)^{-n / 2} e^{-f}$. Then

$$
\left(\partial_{t}+\triangle\right) u=\left(-\frac{n}{2 \sigma}-\partial_{t} f-\triangle f+|\nabla f|^{2}\right) u=R u,
$$

and hence $u$ is again a solution of the adjoint heat equation $\square^{*} u=0$.

We have thus seen that for every gradient soliton the potential function $f$ leads in a natural way to a solution of the adjoint heat equation. This allows us to deduce properties for Ricci solitons from properties of heat equations.

Remark. In this chapter, all manifolds in sight have been assumed compact and without boundary. But the three propositions 1.14-1.16 also hold in the case of noncompact gradient

solitons. Especially in the steady and expanding case this is important, since there the only compact solitons are Einstein manifolds.

\section{Differential Harnack inequalities}

We begin this chapter by recalling some of the classical theory about linear parabolic differential equations. Let $M$ be a compact Riemannian manifold with boundary $\partial M$, where in many cases we will assume that $M$ is closed, i.e. $\partial M=\emptyset$. Let $U$ denote the interior of $\mathrm{M}$, $U:=M \backslash \partial M$, and fix $T>0$. 
Definition 2.1 (parabolic cylinder)

The parabolic cylinder above $U$ is the set $U_{T}:=U \times(0, T]$, its parabolic boundary is defined as

$$
\partial M_{T}:=\overline{U_{T}} \backslash U_{T}=(M \times[0, T]) \backslash(U \times(0, T]) .
$$

Note that the parabolic boundary $\partial M_{T}$ consists of the sides $\partial M \times[0, T]$ and the bottom $M \times\{t=0\}$, but does not contain the top $U \times\{t=T\}$, which belongs to the parabolic interior. For closed manifolds $M$, the parabolic boundary $\partial M_{T}$ is simply $M \times\{t=0\}$.

The following schematic picture illustrates the idea of this cylinder.

Let $L$ denote a second order partial differential operator on $U_{T}$ of the form

$$
L u=-a^{i j}(x, t) \nabla_{i} \nabla_{j} u+b^{i}(x, t) \nabla_{i} u,
$$

where $a^{i j}=a^{j i}$, and $\nabla$ is the Levi-Cività connection. Moreover, let $q=q(x, t) \geq 0$ be a non-negative function on $U_{T}$.

Definition 2.2 (uniformly parabolic operator)

We say that the partial differential operators $\left(\partial_{t}+L\right)$ and $\left(\partial_{t}+L+q\right)$ are uniformly parabolic, if there exists a constant $\lambda>0$, such that

$$
a^{i j}(x, t) \xi_{i} \xi_{j} \geq \lambda|\xi|^{2}, \forall(x, t) \in U_{T}, \forall \xi=\left(\xi_{i}\right) \in \mathbb{R}^{n} .
$$

In this case, for each fixed time $0<t \leq T$, the operators $L$ and $(L+q)$ are uniformly elliptic in the spatial variable $x$.

We will always assume that $a^{i j}, b^{i}$ and $q$ are $C^{\infty}$-functions on $U_{T}, \forall i, j \in\{1, \ldots, n\}$, and $u: \overline{U_{T}} \rightarrow \mathbb{R}$ is a $C^{\infty}$-solution of

$$
\left(\partial_{t}+L+q\right) u=0 \text { in } U_{T} .
$$

Remark. In PDE theory, one shows that there is a (unique) $C^{\infty}$-solution of (2.1) under the additional assumptions $u=0$ on $\partial M \times[0, T]$ and $u=u_{0}$ on $U \times\{t=0\}$ for smooth $u_{0}$ which satisfy some compatibility conditions, cf. [11], chapter 7.1, theorem 7. For closed $M$ there are no compatibility conditions. We are actually not interested in existence and regularity questions in this book and we never need infinitely many derivatives. But if $u$ is at least $C^{4}$ in the $x$-variable and $C^{2}$ in the $t$ variable, and these derivatives have a continuous extension up to the boundary, we can skip a lot of technical details, which distract from the geometric results.

One obvious example for a parabolic operator is $a^{i j}=g^{i j}$, the Riemannian metric, and $b^{i} \equiv 0 \forall i$, in which case $L=-\triangle$ and the operator $\left(\partial_{t}+L\right)$ is the heat operator $\square=\partial_{t}-\triangle$ from chapter one.

The classical Harnack inequality says that for a solution of (2.1) with $u \geq 0$ in $U_{T}, V \Subset U$ connected and $0<t_{1}<t_{2} \leq T$, there exists a constant $C$, which depends only on $V, t_{1}, t_{2}, q$ and the coefficients of $L$, such that

$$
\sup _{V} u\left(\cdot, t_{1}\right) \leq C \inf _{V} u\left(\cdot, t_{2}\right) .
$$


In particular, if $M$ is closed and connected, we find that a non-negative solution $u \in C^{\infty}(M \times$ $[0, T])$ of $\square u=0$ satisfies

$$
\sup _{M} u\left(\cdot, t_{1}\right) \leq C \inf _{M} u\left(\cdot, t_{2}\right),
$$

where $C$ depends only on $t_{1}, t_{2}$ and the geometry of $M$.

As an immediate consequence of this, if $u\left(x_{0}, t_{0}\right)=0$ for some $x_{0} \in M, t_{0}>0$, then $u \equiv 0$ on $M \times\left[0, t_{0}\right]$. Conversely, if $u(\cdot, 0) \geq 0$ with $u\left(x_{0}, 0\right)>0$ for some $x_{0} \in M$, then at any $t>0$ there holds $u(x, t)>0$ for all $x \in M$. That is, we have infinite propagation speed. This phenomenon is a special case of the following more general result.

Proposition 2.3 (strong maximum principle)

Assume $u \in C^{\infty}\left(U_{T}\right)$ and $q \geq 0$ in $U_{T}, U$ connected. Then

i) If $u$ is a subsolution of (2.1), i.e. $\left(\partial_{t}+L+q\right) u \leq 0$ in $U_{T}$, and if $u$ attains a nonnegative absolute maximum over $\overline{U_{T}}$ at an interior point $\left(x_{0}, t_{0}\right) \in U_{T}$, then $u$ is constant on $U_{t_{0}}$.

ii) If $u$ is a supersolution of (2.1), i.e. $\left(\partial_{t}+L+q\right) u \geq 0$ in $U_{T}$, and if $u$ attains a non-positive absolute minimum over $\overline{U_{T}}$ at an interior point $\left(x_{0}, t_{0}\right) \in U_{T}$, then $u$ is constant on $U_{t_{0}}$.

Proof. See [11], chapter 7.1, theorem 12.

The disadvantage is that we only get the qualitative information $u>0$ for all $t>0$, but sometimes one wishes a quantitative lower estimate. Equation (2.2) gives such a lower bound, but the classical results (as found in [11], [24]) have the disadvantage that the geometric dependency of $C$ is complicated and sometimes unclear.

Peter Li and Shing Tung Yau established a pointwise gradient estimate, the so-called Li-Yau differential Harnack inequality, which implies a classical Harnack inequality by integrating along a path. Being a pointwise property of solutions $u$, such a Li-Yau type gradient inequality can also be used to analyze the function $u$ locally. We will start this chapter with a special case of Li and Yau's result, cf. [22].

Richard Hamilton proved a matrix version of the Li-Yau inequality under slightly different assumptions in [17]. It is worth pointing out that in order to prove this, he had to develop a maximum principle for systems first. Hamilton then found a nonlinear analog for the Ricci flow case in [18]. These estimates for the Ricci flow will be our main interest in this chapter. However, no attempt has been made to reprove Hamilton's Harnack inequalities for the Ricci flow here, but rather to give a heuristic motivation for the results and establish the relation between them and the Harnack inequality for the heat equation.

\subsection{The Li-Yau Harnack inequality}

Following Li and Yau's paper [22], we first prove a technical result.

Lemma 2.4 (Li-Yau, lemma 1.1 of [22])

Let $f(x, t)$ be a smooth function on $M \times[0, T]$ satisfying $\partial_{t} f=\Delta f+|\nabla f|^{2}$, and define 
$F:=t\left(|\nabla f|^{2}-\partial_{t} f\right)=-t \triangle f$. Then $F$ satisfies the inequality

$$
\left(\triangle-\partial_{t}\right) F \geq-2\langle\nabla F, \nabla f\rangle-2 K t|\nabla f|^{2}-\frac{1}{t} F+\frac{2}{n t} F^{2},
$$

where $-K$ is a lower bound on the Ricci curvature, with $K \geq 0$.

Proof. We first derive a lower bound for $\triangle|\nabla f|^{2}=2\langle\Delta \nabla f, \nabla f\rangle+2\left\langle\nabla^{2} f, \nabla^{2} f\right\rangle$. Note that

$$
\begin{aligned}
\langle\triangle \nabla f, \nabla f\rangle & =g^{i j} g^{k l} \nabla_{k} \nabla_{l} \nabla_{i} f \nabla_{j} f \\
& =g^{i j} g^{k l} \nabla_{i} \nabla_{k} \nabla_{l} f \nabla_{j} f+g^{i j} g^{k l} R_{k i l p} \nabla_{p} f \nabla_{j} f \\
& =g^{i j} \nabla_{i}(\triangle f) \nabla_{j} f+R_{i p} \nabla_{p} f \nabla_{i} f \\
& =\langle\nabla \triangle f, \nabla f\rangle+\operatorname{Ric}(\nabla f, \nabla f) .
\end{aligned}
$$

We also have

$$
(\triangle f)^{2} \leq\left|g^{i j}\right|^{2}\left|\nabla_{i} \nabla_{j} f\right|^{2}=n|\operatorname{Hess}(f)|^{2},
$$

and putting this together gives

$$
\begin{aligned}
\triangle|\nabla f|^{2} & =2\langle\nabla \triangle f, \nabla f\rangle+2 \operatorname{Ric}(\nabla f, \nabla f)+2|\operatorname{Hess}(f)|^{2} \\
& \geq 2\langle\nabla \triangle f, \nabla f\rangle-2 K|\nabla f|^{2}+\frac{2}{n}(\triangle f)^{2} .
\end{aligned}
$$

Hence we get

$$
\begin{aligned}
\triangle F & =t\left(\triangle|\nabla f|^{2}-\triangle\left(\partial_{t} f\right)\right) \\
& \geq t\left(2\langle\nabla \triangle f, \nabla f\rangle-2 K|\nabla f|^{2}+\frac{2}{n}(\triangle f)^{2}-\partial_{t}(\triangle f)\right) \\
& =2 t\left\langle\nabla\left(\frac{-F}{t}\right), \nabla f\right\rangle-2 K t|\nabla f|^{2}+\frac{2 t}{n}\left(\frac{-F}{t}\right)^{2}-t \partial_{t}\left(\frac{-F}{t}\right) \\
& =-2\langle\nabla F, \nabla f\rangle-2 K t|\nabla f|^{2}-\frac{1}{t} F+\frac{2}{n t} F^{2}+\partial_{t} F .
\end{aligned}
$$

Subtracting $\partial_{t} F$ on both sides yields the desired inequality.

To formulate the Harnack inequality in the case where $\partial M \neq \emptyset$, we need the second fundamental form on a submanifold $N$ of $M$ with co-dimension 1, i.e. an $(n-1)$-dimensional submanifold of the $n$-manifold $M$. Let $\nu \in C^{\infty}\left(N, \mathbb{R}^{n}\right)$, with $|\nu(p)| \equiv 1$ and $\nu(p) \in T_{p} N^{\perp}:=$ $\left\{\mu \in T_{p} M \mid\langle\mu, v\rangle=0, \forall v \in T_{p} N\right\} \subset T_{p} M$, be a normal vector field to $N$ in $T M$. Then the second fundamental form $I I_{p}$ at $p \in N$ with respect to $\nu$ is given by

$$
I I_{p}(v, w):=-\langle d \nu(p) v, w\rangle, \quad v, w \in T_{p} M .
$$

Choose a moving frame $\left\{e_{1}, \ldots, e_{n}\right\}$, where $e_{n}(p)=\nu(p)$ and $\left\{e_{1}(p), \ldots, e_{n-1}(p)\right\}$ is a basis for $T_{p} N$. Then $[\nu, v]=0$ for $v \in \Gamma(T N)$ and hence we see that

$$
I I_{p}(v, w)=-\left\langle\nabla_{\nu(p)} v, w\right\rangle .
$$

We now choose $N=\partial M$ and let $\nu$ be the outward pointing unit normal vector to $\partial M$. We call the boundary of $M$ convex, if the second fundamental form of $\partial M$ with respect to $\nu$ is non-negative, $I I_{p} \geq 0, \forall p \in \partial M$. 
Proposition 2.5 (Li-Yau differential Harnack inequality)

Let $M$ be a compact manifold with non-negative Ricci curvature and convex boundary if $\partial M \neq \emptyset$. Let $u(x, t)$ be a positive solution of the heat equation $\square u=0$ with Neumann boundary condition $\frac{\partial u}{\partial \nu}=0$ on $\partial M \times[0, T]$. Then $u$ satisfies the estimate

$$
H=\frac{\partial_{t} u}{u}-\frac{|\nabla u|^{2}}{u^{2}}+\frac{n}{2 t} \geq 0 \quad \text { on } M \times(0, T] .
$$

Proof. Set $f=\log u$. Note that $0=\left(\partial_{t}-\triangle\right) u=\left(\partial_{t} f-\triangle f-|\nabla f|^{2}\right) u$ and hence $f$ satisfies the assumption of lemma 2.4. We also have

$$
\frac{\partial_{t} u}{u}-\frac{|\nabla u|^{2}}{u^{2}}=\partial_{t} f-|\nabla f|^{2}=-\frac{F}{t},
$$

where $F=t\left(|\nabla f|^{2}-\partial_{t} f\right)$ as in lemma 2.4. The claim is now equivalent to the assertion that $F \leq \frac{n}{2}$, which we will now prove with the inequality we found for $F$, namely

$$
\left(\triangle-\partial_{t}\right) F \geq-2\langle\nabla F, \nabla f\rangle+\frac{2}{n t} F\left(F-\frac{n}{2}\right)
$$

in our case where $K=0$. If the assertion was not true, then there would be a maximum point $\left(x_{0}, t_{0}\right)$ of $F$ on $M \times[0, T]$ where $F\left(x_{0}, t_{0}\right)>\frac{n}{2}>0$. Because $F(x, 0)=0, \forall x \in M, t_{0}$ must be strictly positive. If $x_{0}$ is an interior point of $M$, i.e. $\left(x_{0}, t_{0}\right) \in U_{T}$, then obviously

$$
\nabla F\left(x_{0}, t_{0}\right)=0, \quad \triangle F\left(x_{0}, t_{0}\right) \leq 0, \quad \partial_{t} F\left(x_{0}, t_{0}\right) \geq 0 .
$$

But plugging this into (2.5), gives the contradiction

$$
0 \geq\left(\triangle-\partial_{t}\right) F\left(x_{0}, t_{0}\right) \geq 0+\frac{2}{n t} F\left(x_{0}, t_{0}\right)\left(F\left(x_{0}, t_{0}\right)-\frac{n}{2}\right)>0 .
$$

Hence $x_{0}$ can only be on $\partial M$. In this case the strong maximum principle applied to $F$ implies $\frac{\partial F}{\partial \nu}\left(x_{0}, t_{0}\right)>0$. However in the moving frame described above

$$
\begin{aligned}
\frac{\partial F}{\partial \nu}(p, t) & =t \nabla_{n}\left(|\nabla f|^{2}-\partial_{t} f\right)=2 t \sum_{k=1}^{n} \nabla_{n} \nabla_{k} f \nabla_{k} f-\partial_{t}\left(\nabla_{n} f\right) \\
& =2 t \sum_{k=1}^{n-1} \nabla_{n} \nabla_{k} f \nabla_{k} f=-2 t I I_{p}(\nabla f, \nabla f)
\end{aligned}
$$

where we used the Neumann boundary condition $\nabla_{n} f=0$ on $\partial M$ and the expression for the second fundamental form we derived above. Now because the boundary is convex, and $t_{0}>0$ we get

$$
\frac{\partial F}{\partial \nu}\left(x_{0}, t_{0}\right)=-2 t_{0} I I_{x_{0}}(\nabla f, \nabla f) \leq 0,
$$

which is a contradiction. Hence $F \leq \frac{n}{2}$ on $M \times(0, T]$, and the inequality is proved.

Corollary 2.6 (quadratic version)

Let $M, u$ be as in proposition 2.5. Then for any vector field $V$ on $M$, we have

$$
H(V):=\partial_{t} u+\frac{n}{2 t} u+2\langle\nabla u, V\rangle+u|V|^{2} \geq 0 \quad \text { on } M \times(0, T] .
$$


Proof. We search for the vector field which minimizes this expression. Take a variation $V^{s}$ of $V=V^{0}$, with $\delta V=\left.\partial_{s}\right|_{s=0} V^{s}$. The minimizing $V^{0}$ must satisfy

$$
\left.\partial_{s}\right|_{s=0}\left(H\left(V^{s}\right)\right)=2\langle\nabla u, \delta V\rangle+2 u\left\langle V^{0}, \delta V\right\rangle=0 .
$$

Hence $V^{0}=-\frac{\nabla u}{u}$. Plugging this into the definition of $H$ gives

$$
\begin{aligned}
H\left(V^{0}\right) & =\partial_{t} u+\frac{n}{2 t} u-2\left\langle\nabla u, \frac{\nabla u}{u}\right\rangle+u\left|\frac{\nabla u}{u}\right|^{2} \\
& =\partial_{t} u-\frac{|\nabla u|^{2}}{u}+\frac{n}{2 t} u,
\end{aligned}
$$

which is exactly the Li-Yau Harnack expression multiplied by $u$. This proves that $H(V) \geq$ $H\left(V^{0}\right) \geq 0$.

Finally, we want to integrate the differential Harnack inequality along a path to get a classical Harnack type estimate. Therefore, notice that the Li-Yau Harnack inequality (2.4) is equivalent to

$$
\partial_{t} \log u \geq|\nabla \log u|^{2}-\frac{n}{2 t} .
$$

So on a path $\gamma(t)$, we compute

$$
\begin{aligned}
\frac{d}{d t} \log u(\gamma(t), t) & =\partial_{t} \log u+\nabla \log u \cdot \partial_{t} \gamma(t) \\
& \geq|\nabla \log u|^{2}-\frac{n}{2 t}+\nabla \log u \cdot \dot{\gamma} \\
& =\left|\nabla \log u+\frac{\dot{\gamma}}{2}\right|^{2}-\frac{1}{4}|\dot{\gamma}|^{2}-\frac{n}{2 t} \\
& \geq-\frac{1}{4}|\dot{\gamma}|^{2}-\frac{n}{2 t} .
\end{aligned}
$$

If $\gamma:\left[t_{1}, t_{2}\right] \rightarrow M$ with $\gamma\left(t_{1}\right)=x_{1}$ and $\gamma\left(t_{2}\right)=x_{2}$, then

$$
\begin{aligned}
\log \left(\frac{u\left(x_{2}, t_{2}\right)}{u\left(x_{1}, t_{1}\right)}\right) & =\left.\log u(\gamma(t), t)\right|_{t_{1}} ^{t_{2}}=\int_{t_{1}}^{t_{2}} \frac{d}{d t} \log u(\gamma(t), t) d t \\
& \geq \int_{t_{1}}^{t_{2}}\left(-\frac{1}{4}|\dot{\gamma}|^{2}-\frac{n}{2 t}\right) d t \\
& =-\frac{n}{2} \log \left(\frac{t_{2}}{t_{1}}\right)-\int_{t_{1}}^{t_{2}} \frac{1}{4}|\dot{\gamma}|^{2} d t .
\end{aligned}
$$

By exponentiating, we get

$$
\frac{u\left(x_{2}, t_{2}\right)}{u\left(x_{1}, t_{1}\right)} \geq\left(\frac{t_{2}}{t_{1}}\right)^{-n / 2} \exp \left\{-\int_{t_{1}}^{t_{2}} \frac{1}{4}|\dot{\gamma}|^{2} d t\right\} .
$$

This proves the following corollary.

Corollary 2.7 (integrated version)

Under the same conditions as in proposition 2.5, a positive solution $u$ of $\square u=0$ satisfies the inequality

$$
u\left(x_{2}, t_{2}\right) \geq u\left(x_{1}, t_{1}\right)\left(\frac{t_{2}}{t_{1}}\right)^{-n / 2} e^{-L\left(x_{1}, x_{2}, t_{2}-t_{1}\right)},
$$


where

$$
L\left(x_{1}, x_{2}, t_{2}-t_{1}\right):=\inf _{\gamma \in \Gamma}\left\{\frac{1}{2\left(t_{2}-t_{1}\right)} \int_{0}^{1} \frac{1}{2}|\dot{\gamma}|^{2} d t\right\}
$$

with

$$
\Gamma=\left\{\gamma:[0,1] \rightarrow M \mid \gamma(0)=x_{1}, \gamma(1)=x_{2}\right\}
$$

Note that in the case where $M$ is a geodesic manifold, i.e. every pair of points $p$ and $q$ in $M$ can be joined by a minimizing geodesic, we have

$$
L\left(x_{1}, x_{2}, t_{2}-t_{1}\right)=\frac{E\left(x_{1}, x_{2}\right)}{2\left(t_{2}-t_{1}\right)}=\frac{d^{2}\left(x_{1}, x_{2}\right)}{4\left(t_{2}-t_{1}\right)},
$$

where $E\left(x_{1}, x_{2}\right)$ is the classical energy of such a minimizing geodesic between $x_{1}$ and $x_{2}$.

On flat $\mathbb{R}^{n}$, a minimizing geodesic is of course a straight line, hence $|\dot{\gamma}|^{2}=\left|x_{2}-x_{1}\right|^{2}$ and

$$
L\left(x_{1}, x_{2}, t_{2}-t_{1}\right)=\frac{\left|x_{2}-x_{1}\right|^{2}}{4\left(t_{2}-t_{1}\right)}
$$

In this case, the inequality in the corollary becomes an equality for the heat kernel $\bar{u}(x, t)=$ $(4 \pi t)^{-n / 2} e^{-|x|^{2} / 4 t}$ at $x_{1}=x_{2}=0$. Moreover, the function $u(x, t)=(4 \pi t)^{-n / 2} e^{-L\left(x_{1}, x, t-t_{1}\right)}$ then satisfies the heat equation $\square u=0$ for fixed $x_{1}, t_{1}$.

One can show something similar for general manifolds with Ric $\geq 0$ (but with the inequality $\square u \leq 0$ instead of an equality). We will do that in chapter three. Moreover, Perelman's $\mathcal{L}$-functional can be seen as an analog of the above energy functional under backwards Ricci flow. However this needs a lot of long and technical computations. We will discuss this functional in chapter four.

\subsection{Hamilton's matrix Harnack inequality}

To prove Hamilton's matrix version of the Li-Yau result, one needs a version of the maximum principle which holds for systems of equations. We closely follow Hamilton's idea, cf. [14]. Let $f=\left\{f^{\alpha}\right\}$ be a system of $m$ functions on the compact manifold $M$, satisfying the non-linear heat equation

$$
\partial_{t} f=\triangle f+\phi(f), \quad f=f_{0} \quad \text { at } t=0 .
$$

where $\phi: U \subset \mathbb{R}^{m} \rightarrow \mathbb{R}^{m}$ is a smooth vector field. The basic idea is that the effect of the heat equation is to average out the system and hence, if $f$ lies in a convex set $X \subset U$ at $t=0$, it will remain there, unless it is moved out by $\phi$.

Suppose (2.8) has a smooth solution for $t \in[0, T]$. To study the PDE, we first look at the ODE

$$
\partial_{t} f=\phi(f), \quad f=f_{0} \quad \text { at } t=0 .
$$

Let $X \subset U$ be a closed convex subset. Define the tangent cone $T_{k} X$ to $X$ at $k \in \partial X$ as the intersection of all closed half-spaces containing $X$ with $k$ on the boundary of the half-space. This is the smallest closed convex cone with vertex at $k$ which contains the set $X$. Hamilton proved the following proposition in [14]. 
Proposition 2.8 (maximum principle for systems)

Let $X$ be a closed and convex subset of $U$.

i) Solutions of the $O D E$ (2.9) which start in $X$ will remain in $X$ if and only if for all $k \in \partial X$ we have $\phi(k) \in T_{k} X$.

ii) If the solution of the ODE (2.9) remains in $X$, then so does the solution of the heat equation (2.8).

Note that the path of solutions does not change when we multiply $\phi(f)$ with a cutoff function which equals 1 on a very large ball. Hence we may always assume $X$ to be compact by intersecting it with an even larger ball on whose complement the cutoff function disappears.

The idea of the proof is to reduce the problem to the one-dimensional case. One does that with linear functionals $\ell$ on $\mathbb{R}^{m}$ : We say $\ell$ is a support function for $X$ at $f \in \partial X$ (or $\ell \in S_{f} X$ for short) if $\|\ell\|=1$ and $\ell(f) \geq \ell(k)$ for all $k \in X$. Then

$$
\phi(f) \in T_{f} X \Leftrightarrow \ell(\phi(f)) \leq 0, \quad \forall \ell \in S_{f} X .
$$

Define the distance function

$$
s(f)=\operatorname{dist}(f, X)= \begin{cases}\sup \left\{\ell(f-k) \mid k \in \partial X, \ell \in S_{k} X\right\} & \text { if } f \notin X, \\ 0 & \text { if } f \in X .\end{cases}
$$

To estimate the derivative of $s(f)$ we need the following lemma.

\section{Lemma 2.9}

Let $g$ be a smooth function of $t \in \mathbb{R}$ and $y \in \mathbb{R}^{m}$. Let $f(t)=\sup \{g(t, y) \mid y \in Y\}$ where $Y \subset \mathbb{R}^{m}$ is compact. Then $f(t)$ is Lipschitz (and hence differentiable almost everywhere) and with $Y(t)=\{y \in Y \mid g(t, y)=f(t)\}$ we have

$$
\partial_{t} f(t) \leq \sup \left\{\partial_{t} g(t, y) \mid y \in Y(t)\right\} .
$$

Proof. Choose a sequence of times $t_{j}$ decreasing to $t_{0}$ such that

$$
\lim _{t_{j} \searrow t_{0}} \frac{f\left(t_{j}\right)-f\left(t_{0}\right)}{t_{j}-t_{0}}=\limsup _{t \searrow t_{0}} \frac{f(t)-f\left(t_{0}\right)}{t-t_{0}} .
$$

Because $Y$ is compact, we can choose $y_{j} \in Y$ with $f\left(t_{j}\right)=g\left(t_{j}, y_{j}\right)$. Moreover there is a point $y_{0} \in Y$ with $y_{j} \rightarrow y_{0}$ for a subsequence. Without loss of generality, the whole sequence converges to $y_{0}$. Since $g$ is continuous, $f\left(t_{0}\right)=g\left(t_{0}, y_{0}\right)$ and $y_{0} \in Y(t)$. Moreover $g\left(t_{0}, y_{j}\right) \leq g\left(t_{0}, y_{0}\right)$ yields

$$
f\left(t_{j}\right)-f\left(t_{0}\right) \leq g\left(t_{j}, y_{j}\right)-g\left(t_{0}, y_{j}\right),
$$

and by the mean value theorem there exists $\xi_{j} \in\left[t_{0}, t_{j}\right]$ with

$$
\frac{g\left(t_{j}, y_{j}\right)-g\left(t_{0}, y_{j}\right)}{t_{j}-t_{0}}=\partial_{t} g\left(\xi_{j}, y_{j}\right) .
$$

Now because $\xi_{j} \rightarrow t_{0}$ too, and because $g$ is smooth, we find

$$
\lim _{t_{j} \searrow t_{0}} \frac{f\left(t_{j}\right)-f\left(t_{0}\right)}{t_{j}-t_{0}} \leq \lim _{j \rightarrow \infty} \frac{g\left(t_{j}, y_{j}\right)-g\left(t_{0}, y_{j}\right)}{t_{j}-t_{0}}=\lim _{j \rightarrow \infty} \partial_{t} g\left(\xi_{j}, y_{j}\right)=\partial_{t} g\left(t_{0}, y_{0}\right),
$$

which proves the lemma. 
We have now the necessary material to prove the maximum principle for systems.

Proof of proposition 2.8. i) If $\phi(f) \notin T_{f} X$, then with (2.10) there is an $\ell \in S_{f} X$ such that $\ell(\phi(f))>0$. We find

$$
\partial_{t} \ell(f)=\ell\left(\partial_{t} f\right)=\ell(\phi(f))>0
$$

and hence $\ell(f)$ is increasing and $f$ can not stay in $X$. For the converse, suppose $\ell(\phi(k)) \leq$ $0, \forall k \in \partial X, \forall \ell \in S_{k} X$. With lemma 2.9 applied to

$$
g=g(t, k, \ell)=\ell(f(t)-k) \text { and } Y=\left\{(k, \ell) \mid k \in \partial X, \ell \in S_{k} X\right\}
$$

and with the definition of $s(f)$ we get

$$
\begin{aligned}
\partial_{t} s(f) & \leq \sup \left\{\partial_{t} \ell(f-k)\right\}=\sup \{\ell(\phi(f))\} \\
& \leq \sup \{\ell(\phi(f))-\ell(\phi(k))\},
\end{aligned}
$$

where the supremum is taken over $k \in \partial X$ and $\ell \in S_{k} X$ with $s(f)=\ell(f-k)$. Because $X$ is convex, $k$ must be the unique closest point on $\partial X$ to $f$, and $\ell$ must be the functional with gradient in the direction $f-k$. Because $\phi$ is smooth, $|\phi(f)-\phi(k)| \leq C|f-k|=C s(f)$, and so (2.12) becomes $\partial_{t} s(f) \leq C s(f)$. Since $s(f)=0$ at $t=0$, it must remain 0 .

ii) In the PDE case, the computations are similar, but we also have to take the supremum over all $x \in M$. Note that $s(t)=\sup _{x \in M} s(f(x, t))$ satisfies

$$
\partial_{t} s(t) \leq \sup \left\{\partial_{t} \ell(f(x, t)-k)\right\}=\sup \{\ell(\triangle f)+\ell(\phi(f))\},
$$

the supremum now being taken over all $x \in M, k \in \partial X$ and $\ell \in S_{k} X$ with $s(t)=\ell(f(x, t)-$ $k)$. Because $\ell(f(x, t))$ has its maximum at $x$, the term $\ell(\triangle f)=\Delta \ell(f)$ is non-positive. So, as before, we get

$$
\partial_{t} s(t) \leq \sup \{\ell(\phi(f))\} \leq|\phi(f)-\phi(k)| \leq C|f-k|=C s(t),
$$

and since $s(t)=0$ at $t=0$, it must remain 0 .

An immediate consequence is the following.

Corollary 2.10 (maximum principle for matrices)

Let $M$ be compact. Let $M_{i j}(x, t)$ be a symmetric $(n \times n)$-matrix which depends smoothly on $t \in[0, T]$ and $x \in M$. Suppose $M_{i j}$ satisfies the heat equation

$$
\partial_{t} M_{i j}=\triangle M_{i j}+\phi\left(M_{i j}\right)
$$

where $\phi\left(M_{i j}\right)(v, v) \geq 0$ for all zero-eigenvectors $v$ of $M_{i j}$. Then if $M_{i j} \geq 0$ at $t=0$, it remains so for all $t \in[0, T]$.

Remark. If the corollary is true, then it obviously also holds for supersolutions which satisfy $\partial_{t} M_{i j} \geq \triangle M_{i j}+\phi\left(M_{i j}\right)$.

With the help of this corollary we can now prove Hamilton's matrix version of the Li-Yau result. To shorten the proof, we assume that $u$ is smooth and solves the heat equation up to the boundary $M \times\{t=0\}$, while Hamilton [17] proves the inequality under more general assumptions on $u$. 
Proposition 2.11 (Hamilton's matrix Harnack inequality)

Let $M$ be a closed Riemannian manifold and $u \in C^{\infty}(M \times[0, T])$ a positive solution to the heat equation $\square u=0$. Suppose $M$ is Ricci parallel and has weakly positive sectional curvatures: $\nabla \operatorname{Ric}=0$ and $\langle\operatorname{Rm}(v, w) w, v\rangle=R_{i j k l} v_{i} w_{j} v_{k} w_{l} \geq 0$ for all $v, w \in \Gamma(T M)$. Then

$$
H_{i j}:=\nabla_{i} \nabla_{j} u-\frac{\nabla_{i} u \nabla_{j} u}{u}+\frac{u}{2 t} g_{i j} \geq 0 \quad \text { on } M \times(0, T] .
$$

Proof. Because $u, \nabla u$ and $\nabla^{2} u$ are bounded on $M \times[0, T]$, the inequality $H_{i j} \geq 0$ obviously holds for $t$ small enough. If we can show that $H_{i j}$ satisfies a heat equation of the form (2.13), with $\phi\left(H_{i j}\right) \geq 0$ whenever $H_{i j} \geq 0$, then we are done. Set

$$
L_{i j}:=\nabla_{i} \nabla_{j} u-\frac{\nabla_{i} u \nabla_{j} u}{u}=H_{i j}-\frac{u}{2 t} g_{i j} .
$$

We first calculate $(\star):=\triangle L_{i j}-\triangle \nabla_{i} \nabla_{j} u$. We get

$$
\begin{aligned}
(\star)= & -g^{k \ell} \nabla_{k}\left(\frac{\nabla_{\ell} \nabla_{i} u \nabla_{j} u+\nabla_{i} u \nabla_{\ell} \nabla_{j} u}{u}-\frac{\nabla_{i} u \nabla_{j} u \nabla_{\ell} u}{u^{2}}\right) \\
= & -g^{k \ell}\left(\frac{\nabla_{k} \nabla_{i} \nabla_{\ell} u \nabla_{j} u}{u}+\frac{\nabla_{i} u \nabla_{k} \nabla_{j} \nabla_{\ell} u}{u}-\frac{\nabla_{i} u \nabla_{j} u \nabla_{k} \nabla_{\ell} u}{u^{2}}\right) \\
& -\frac{2}{u}\left(\nabla_{k} \nabla_{i} u-\frac{\nabla_{k} u \nabla_{i} u}{u}\right) \cdot\left(\nabla_{k} \nabla_{j} u-\frac{\nabla_{k} u \nabla_{j} u}{u}\right) \\
= & -\left(\frac{\nabla_{i}(\triangle u) \nabla_{j} u}{u}+\frac{\nabla_{i} u \nabla_{j}(\triangle u)}{u}-\frac{\nabla_{i} u \nabla_{j} u \triangle u}{u^{2}}\right) \\
& -g^{k \ell}\left(R_{k i \ell p} \frac{\nabla_{p} u \nabla_{j} u}{u}+R_{k j \ell p} \frac{\nabla_{p} u \nabla_{i} u}{u}\right)-\frac{2}{u} L_{k i} L_{k j} \\
= & -\partial_{t}\left(\frac{\nabla_{i} u \nabla_{j} u}{u}\right)-R_{i p} \frac{\nabla_{p} u \nabla_{j} u}{u}-R_{j p} \frac{\nabla_{p} u \nabla_{i} u}{u}-\frac{2}{u} L_{i j}^{2} .
\end{aligned}
$$

Using (1.13), we also have

$$
\begin{aligned}
\partial_{t}\left(\nabla_{i} \nabla_{j} u\right)= & \nabla_{i} \nabla_{j}(\triangle u)=g^{k \ell} \nabla_{i} \nabla_{j} \nabla_{k} \nabla_{\ell} u \\
= & g^{k \ell} \nabla_{i} \nabla_{k} \nabla_{j} \nabla_{\ell} u-\nabla_{i}\left(R_{j p} \nabla_{p} u\right) \\
= & g^{k \ell} \nabla_{k} \nabla_{i} \nabla_{\ell} \nabla_{j} u-\nabla_{i}\left(R_{j p} \nabla_{p} u\right) \\
& -R_{i p} \nabla_{p} \nabla_{j} u+R_{i k j p} \nabla_{k} \nabla_{p} u \\
= & \triangle \nabla_{i} \nabla_{j} u-\nabla_{i} R_{j p} \nabla_{p} u-R_{j p} \nabla_{p} \nabla_{i} u-R_{i p} \nabla_{p} \nabla_{j} u \\
& +R_{i k j p} \nabla_{k} \nabla_{p} u+\nabla_{k}\left(R_{i k j p} \nabla_{p} u\right) \\
= & \triangle \nabla_{i} \nabla_{j} u-R_{j p} \nabla_{p} \nabla_{i} u-R_{i p} \nabla_{p} \nabla_{j} u+2 R_{i k j p} \nabla_{k} \nabla_{p} u \\
& -\nabla_{i} R_{j p} \nabla_{p} u+\nabla_{p} R_{i j} \nabla_{p} u-\nabla_{j} R_{i p} \nabla_{p} u,
\end{aligned}
$$

where in the last step we used the contracted second Bianchi identity (1.7) to get

$$
\begin{aligned}
\nabla_{k}\left(R_{i k j p} \nabla_{p} u\right) & =\nabla_{k} R_{i k j p} \nabla_{p} u+R_{i k j p} \nabla_{k} \nabla_{p} u \\
& =-\nabla_{k} R_{k i j p} \nabla_{p} u+R_{i k j l} \nabla_{k} \nabla_{l} u \\
& =-\nabla_{j} R_{i p} \nabla_{p} u+\nabla_{p} R_{i j} \nabla_{p} u+R_{i k j l} \nabla_{k} \nabla_{l} u
\end{aligned}
$$


Now we put all this together. We find

$$
\begin{aligned}
\partial_{t} L_{i j}= & \partial_{t}\left(\nabla_{i} \nabla_{j} u\right)-\partial_{t}\left(\frac{\nabla_{i} u \nabla_{j} u}{u}\right) \\
= & \triangle L_{i j}+\frac{2}{u} L_{i j}^{2}+2 R_{i k j \ell} \nabla_{k} \nabla_{\ell} u-R_{i k} L_{j k}-R_{j k} L_{i k} \\
& +\left(\nabla_{p} R_{i j}-\nabla_{j} R_{i p}-\nabla_{i} R_{j p}\right) \nabla_{p} u \\
= & \triangle L_{i j}+\frac{2}{u} L_{i j}^{2}+2 R_{i k j \ell} L_{k \ell}-R_{i k} L_{j k}-R_{j k} L_{i k} \\
& +\frac{2}{u} R_{i k \ell j} \nabla_{k} u \nabla_{\ell} u+\left(\nabla_{p} R_{i j}-\nabla_{j} R_{i p}-\nabla_{i} R_{j p}\right) \nabla_{p} u
\end{aligned}
$$

where the last line is non-negative, because of the assumptions that $M$ is Ricci parallel and has weakly positive sectional curvatures. Thus

$$
\partial_{t} L_{i j} \geq \triangle L_{i j}+\frac{2}{u} L_{i j}^{2}+2 R_{i k j \ell} L_{k \ell}-R_{i k} L_{j k}-R_{j k} L_{i k} .
$$

It is now easy to derive the evolution equation for $H_{i j}=L_{i j}+\frac{u}{2 t} g_{i j}$. With

$$
\triangle H_{i j}=\triangle L_{i j}+\frac{\triangle u}{2 t} g_{i j}
$$

we obtain

$$
\begin{aligned}
\partial_{t} H_{i j} & =\partial_{t} L_{i j}+\frac{\triangle u}{2 t} g_{i j}-\frac{u}{2 t^{2}} g_{i j} \\
& \geq \triangle L_{i j}+\frac{2}{u} L_{i j}^{2}+2 R_{i k j \ell} L_{k \ell}-R_{i k} L_{j k}-R_{j k} L_{i k}+\frac{\triangle u}{2 t} g_{i j}-\frac{u}{2 t^{2}} g_{i j} \\
& =\triangle H_{i j}+\frac{2}{u} L_{i j}^{2}+2 R_{i k j \ell} L_{k \ell}-R_{i k} L_{j k}-R_{j k} L_{i k}-\frac{u}{2 t^{2}} g_{i j} .
\end{aligned}
$$

Note that

$$
2 R_{i k j \ell} \cdot \frac{u}{2 t} g_{k \ell}-R_{i k} \cdot \frac{u}{2 t} g_{j k}-R_{j k} \cdot \frac{u}{2 t} g_{i k}=\frac{u}{2 t}\left(2 R_{i j}-R_{i j}-R_{i j}\right)=0,
$$

and hence

$$
2 R_{i k j \ell} L_{k \ell}-R_{i k} L_{j k}-R_{j k} L_{i k}=2 R_{i k j \ell} H_{k \ell}-R_{i k} H_{j k}-R_{j k} H_{i k}
$$

With this and with

$$
\begin{aligned}
\frac{2}{u} L_{i j}^{2} & =\frac{2}{u} g^{k \ell}\left(H_{i k}-\frac{u}{2 t} g_{i k}\right)\left(H_{j \ell}-\frac{u}{2 t} g_{j \ell}\right) \\
& =\frac{2}{u} H_{i j}^{2}-\frac{2}{t} H_{i j}+\frac{u}{2 t^{2}} g_{i j}
\end{aligned}
$$

we find the desired evolution inequality

$$
\partial_{t} H_{i j} \geq \triangle H_{i j}+\frac{2}{u} H_{i j}^{2}-\frac{2}{t} H_{i j}+2 R_{i k j \ell} H_{k \ell}-R_{i k} H_{j k}-R_{j k} H_{i k} .
$$

This is of the form (2.13) and all the terms $H_{i j}^{2}, R_{i k j \ell} H_{k \ell},-R_{i k} H_{j k}$, and $-R_{j k} H_{i k}$ are non-negative at a zero-eigenvector of $H_{i j}$, which allows us to apply the maximum principle for symmetric matrices. Hence $H_{i j}$ will remain non-negative for all time. 
Remark. The inequality (2.15) proves that if $L_{i j} \geq 0$ at time $t_{0}$ then $L_{i j} \geq 0$ for all $t \geq t_{0}$. However $L_{i j}$ could also start negative and stay negative for all time.

As in the trace case, the matrix Harnack inequality becomes an equality for the heat kernel $\bar{u}=(4 \pi t)^{-n / 2} e^{-|x|^{2} / 4 t}$ on Euclidean space $\mathbb{R}^{n}$.

We can again find a quadratic vector field version of this Harnack inequality:

Corollary 2.12 (quadratic version)

Let $M, u$ be as in proposition 2.11. Then for any vector field $V$ on $M$ we have

$$
H_{i j}(V):=\nabla_{i} \nabla_{j} u+\frac{u}{2 t} g_{i j}+\nabla_{i} u V_{j}+\nabla_{j} u V_{i}+u V_{i} V_{j} \geq 0 \quad \text { on } M \times(0, T] .
$$

Proof. As in corollary 2.6, the minimizing $V^{0}$ must be $V^{0}=-\frac{\nabla u}{u}$ and we get

$$
H_{i j}(V) \geq H_{i j}\left(V^{0}\right)=\nabla_{i} \nabla_{j} u-\frac{\nabla_{i} u \nabla_{j} u}{u}+\frac{u}{2 t} g_{i j} \geq 0 .
$$

We can also use Hamilton's argument to prove the Li-Yau inequality. This gives a better understanding of how the Harnack expression itself evolves in time, and of why we need the assumption of non-negative Ricci curvature. On a closed manifold $M$ and for a positive solution $u$ of the heat equation, we set

$$
H=\partial_{t} u-\frac{|\nabla u|^{2}}{u}+\frac{n}{2 t} u, \quad L=H-\frac{n}{2 t} u .
$$

Then suppose that $H$ is positive for $t$ small enough. With the above computation (2.14) for $\triangle\left(\frac{\nabla_{i} u \nabla_{j} u}{u}\right)$, which does not require bounds on the Ricci curvature, we find

$$
\begin{aligned}
\triangle L-\triangle\left(\partial_{t} u\right) & =g^{i j}\left[-\triangle\left(\frac{\nabla_{i} u \nabla_{j} u}{u}\right)\right] \\
& =-\partial_{t} \frac{|\nabla u|^{2}}{u}-\frac{2}{u} \operatorname{Ric}(\nabla u, \nabla u)-\frac{2}{u}\left|L_{i j}\right|^{2} \\
& \leq-\partial_{t} \frac{|\nabla u|^{2}}{u}-\frac{2}{u} \operatorname{Ric}(\nabla u, \nabla u)-\frac{2}{n u} L^{2},
\end{aligned}
$$

where we used the Cauchy-Schwarz inequality for $L^{2}=\left(g^{i j} L_{i j}\right)^{2} \leq n\left|L_{i j}\right|^{2}$ in the last step. Because $\triangle\left(\partial_{t} u\right)=\partial_{t}(\triangle u)=\partial_{t}\left(\partial_{t} u\right)$, we get

$$
\partial_{t} L \geq \triangle L+\frac{2}{n u} L^{2}+\frac{2}{u} \operatorname{Ric}(\nabla u, \nabla u) .
$$

With $\partial_{t} H=\partial_{t} L-\frac{n}{2 t} \triangle u+\frac{n}{2 t^{2}} u$ and $\triangle H=\triangle L-\frac{n}{2 t} \triangle u$, we finally find

$$
\partial_{t} H \geq \triangle H+\frac{2}{n u} H^{2}-\frac{2}{t} H+\frac{2}{u} \operatorname{Ric}(\nabla u, \nabla u) .
$$

This shows that $H$ is supersolution to a non-linear heat equation (and hence stays positive) exactly under the assumption of non-negative Ricci curvature. 
Remark. In the case of Ric $\geq 0$, we also find that $L=\partial_{t} u-\frac{|\nabla u|^{2}}{u} \geq 0$ is preserved if initially true, exactly as in the matrix case with $L_{i j}$. But again $L$ could also be negative for all time.

Furthermore, we can again derive an integrated version of the matrix Harnack inequality, as for the Li-Yau result. Let $M$ satisfy the assumptions of proposition 2.11 and suppose that $u>0$ is a solution of $\square u=0$. Then by multiplying the Harnack inequality with $\frac{1}{u}$, we get

$$
\begin{aligned}
0 & \leq \frac{1}{u} H_{i j}=\frac{1}{u}\left(\nabla_{i} \nabla_{j} u-\frac{\nabla_{i} u \nabla_{j} u}{u}\right)+\frac{1}{2 t} g_{i j} \\
& =\frac{u \nabla_{i} \nabla_{j} u-\nabla_{i} u \nabla_{j} u}{u^{2}}+\frac{1}{2 t} g_{i j} \\
& =\nabla_{i}\left(\frac{\nabla_{j} u}{u}\right)+\frac{1}{2 t} g_{i j} \\
& =\nabla_{i} \nabla_{j} \log u+\frac{1}{2 t} g_{i j},
\end{aligned}
$$

which is a kind of logarithmic convexity. Hence, along a geodesic $\gamma(s)$ parameterized by arc-length, we get

$$
\partial_{s}^{2} \log u+\frac{1}{2 t} \geq 0
$$

or equivalently

$$
\partial_{s}^{2}\left(\log u+\frac{s^{2}}{4 t}\right) \geq 0
$$

i.e. $h(s, t)=\log u(\gamma(s), t)+\frac{s^{2}}{4 t}$ is convex in $s$. We conclude that for any geodesic $\gamma(s)$ parameterized by arc-length, we have

$$
h(0, t) \leq \frac{1}{2} h\left(s_{0}, t\right)+\frac{1}{2} h\left(-s_{0}, t\right) .
$$

By exponentiation, we find the following result

Corollary 2.13 (integrated version)

Let $M, u$ be as in proposition 2.11 and let $\gamma(s): I \rightarrow M$ be a geodesic parameterized by arc-length. Then for $s_{0}$ with $\pm s_{0} \in I$, we have

$$
u(\gamma(0), t) \leq u\left(\gamma\left(s_{0}\right), t\right)^{1 / 2} u\left(\gamma\left(-s_{0}\right), t\right)^{1 / 2} e^{s^{2} / 4 t} .
$$

\subsection{Harnack inequalities for the Ricci flow}

Remember that the trace and matrix Harnack expressions for the heat equation both vanish on the heat kernel, which we interpret as a gradient expander in the following way. The Euclidean metric is an expanding soliton in the sense of proposition 1.10, with birth time $T=0$ and potential $f(x, t)=-|x|^{2} / 4 t$, i.e. the metric expands along the vector field $X=\nabla f=-\frac{x}{2 t}$ and we have $\Phi_{t}(x)=\frac{x}{\sqrt{t}}$. Motivated by this, when searching for a Harnack estimate for the Ricci flow, we will try to find an expression which vanishes on gradient Ricci expanders.

Recall from the first chapter, proposition 1.16, that such an expanding soliton satisfies the equation $\operatorname{Ric}+\operatorname{Hess}(f)+\frac{g}{2 t}=0$, if we set the birth time $T$ to zero. With $V=\nabla f$ we have

$$
-\nabla_{i} V_{j}=R_{i j}+\frac{g_{i j}}{2 t}
$$


Differentiating and commuting gives

$$
\nabla_{j} R_{i k}-\nabla_{i} R_{j k}=\nabla_{i} \nabla_{j} V_{k}-\nabla_{j} \nabla_{i} V_{k}=R_{i j k l} V_{l}
$$

Then tracing with $g^{i k}$ and applying the twice contracted second Bianchi identity (1.8) yields

$$
\nabla_{j} R-\nabla_{k} R_{j k}=\frac{1}{2} \nabla_{j} R=R_{j \ell} V_{\ell} .
$$

Applying this to $V$ gives our first expression

$$
-\nabla_{i} R V_{i}+2 R_{i j} V_{i} V_{j}=0 .
$$

Differentiating (2.20) again, and tracing with $g^{i k}$ gives

$$
\begin{aligned}
0 & =-g^{i k} \nabla_{k} \nabla_{i} R+2 g^{i k} \nabla_{k} R_{i j} V_{j}+2 g^{i k} R_{i j} \nabla_{k} V_{j} \\
& =-\triangle R+2 \nabla_{i} R_{i j} V_{j}+2 R_{i j} \nabla_{i} V_{j} \\
& =-\triangle R+\nabla_{j} R V_{j}+2 R_{i j} \nabla_{i} V_{j} \\
& =-\triangle R+\nabla_{j} R V_{j}-2|\operatorname{Ric}|^{2}-2 \frac{R}{2 t} \\
& =-\partial_{t} R+\nabla_{i} R V_{i}-\frac{R}{t} .
\end{aligned}
$$

Here, we used (2.19) and the evolution equation for the scalar curvature (1.18) for the last two steps. Now, by subtracting (2.22) from (2.21), we get

$$
H(V)=\partial_{t} R+\frac{R}{t}-2 \nabla_{i} R V_{i}+2 R_{i j} V_{i} V_{j}=0 .
$$

Observe that $H(V)$ is a similar expression to the quadratic version of Hamilton's Harnack for the heat equation in corollary 2.12. To see this claimed similarity even better, we formally take the inequality from corollary 2.12 and plug in $2 R_{i j}$ instead of $u$ (note that $u$ satisfies $\partial_{t} u=\triangle u$ while $R_{i j}$ satisfies $\partial_{t} R_{i j}=\triangle_{L} R_{i j}$ ). With (1.8) we get

$$
\begin{aligned}
0 & \leq \cdot 2 \nabla_{i} \nabla_{j} R_{i j}+\frac{2 R_{i j}}{2 t} g_{i j}+2 \nabla_{i} R_{i j} V_{j}+2 \nabla_{j} R_{i j} V_{i}+2 R_{i j} V_{i} V_{j} \\
& =\nabla_{i} \nabla_{i} R+\frac{R}{t}+\nabla_{j} R V_{j}+\nabla_{i} R V_{i}+2 R_{i j} V_{i} V_{j} \\
& =\triangle R+\frac{R}{t}+2 \nabla_{j} R V_{j}+2 R_{i j} V_{i} V_{j} \\
& \leq \partial_{t} R+\frac{R}{t}+2 \nabla_{i} R V_{i}+2 R_{i j} V_{i} V_{j}=H(-V) .
\end{aligned}
$$

This heuristic argument shows that our $H(V)$ may in fact be the right expression. Note that the Harnack inequality holds for positive solutions $u$ of the heat equation, so here we will probably also have to assume Ric $\geq 0$ to get the Harnack inequality $H(V) \geq 0$.

Moreover, if we do not trace (2.22) and (2.21), we get a matrix expression which vanishes on expanding solitons. This actually turns out to be Hamilton's matrix Harnack expression for the Ricci flow. The similarity to corollary 2.12 can now be seen if we insert Rm instead of Ric or $u$. This also heuristically explains why we have to assume $\mathrm{Rm} \geq 0$ (i.e. weakly positive curvature operator, $R_{i j k \ell} U_{i j} U_{k \ell} \geq 0$ for all two-forms $U$ ) to get a non-negative Harnack expression. Hence the following theorem is not a big surprise anymore. 
Theorem 2.14 (Hamilton's Harnack estimates for the Ricci flow)

Suppose $(M, g)$ is a complete solution to the Ricci flow (1.14) for $t \in[0, T]$. Suppose $g_{i j}$ has a weakly positive curvature operator. Define

$$
M_{i j}=\triangle R_{i j}-\frac{1}{2} \nabla_{i} \nabla_{j} R+2 R_{i k j \ell} R_{k \ell}-R_{i k} R_{j k}+\frac{1}{2 t} R_{i j} .
$$

Then for any vectors $V, W$ and $t>0$ we have

$$
\begin{gathered}
\text { i) } H(V, W):=2 M_{i j} W_{i} W_{j}-4\left(\nabla_{i} R_{j k}\right)\left(V_{i} W_{j}-V_{j} W_{i}\right) W_{k} \\
+2 R_{i j k \ell} V_{i} W_{j} V_{k} W_{\ell} \geq 0 \\
\text { ii) } H(V):=\partial_{t} R+\frac{R}{t}-2 \nabla_{i} R V_{i}+2 R_{i j} V_{i} V_{j} \geq 0 .
\end{gathered}
$$

Proof. The proof is similar to Hamilton's proof of the matrix Harnack estimate for the heat equation, i.e. one shows that the expressions $H(V, W)$ and $H(V)$ are supersolutions to non-linear heat equations which preserve the cone $H(V, W) \geq 0$ or $H(V) \geq 0$, respectively. However the computations are obviously longer and more complicated. We hence skip them here and refer the reader to [17]. Note that one has to substitute $V$ by $-V$ and set $U=V \wedge W$ (i.e. $\left.U_{i j}=\frac{1}{2}\left(V_{i} W_{j}-V_{j} W_{i}\right)\right)$ to get Hamilton's expression as found in [17].

We now want to write this theorem in a different form. Use

$$
\begin{aligned}
H(V, W)= & 2 M_{i j} W_{i} W_{j}-4\left(\nabla_{i} R_{j k}\right)\left(V_{i} W_{j}-V_{j} W_{i}\right) W_{k} \\
& +2 R_{i j k \ell} V_{i} W_{j} V_{k} W_{\ell} \\
= & 2 M(W, W)-4\left(\nabla_{V} \operatorname{Ric}\right)(W, W)+4\left(\nabla_{W} \operatorname{Ric}\right)(V, W) \\
& +2\langle R(V, W) W, V\rangle .
\end{aligned}
$$

Note that with $\partial_{t} R_{i j}=\triangle R_{i j}+2 R_{i k j \ell} R_{k \ell}-2 R_{i k} R_{j k}$ we get

$$
\begin{aligned}
2 M_{i j} & =2 \triangle R_{i j}+4 R_{i k j \ell} R_{k \ell}-2 R_{i k} R_{j k}-\nabla_{i} \nabla_{j} R+\frac{1}{t} R_{i j} \\
& =2 \partial_{t} R_{i j}+2 R_{i k} R_{j k}-\nabla_{i} \nabla_{j} R+\frac{1}{t} R_{i j},
\end{aligned}
$$

and hence

$$
2 M(W, W)=2\left(\partial_{t} \operatorname{Ric}\right)(W, W)+2|\operatorname{Ric}(W, \cdot)|^{2}-\nabla_{W} \nabla_{W} R+\frac{1}{t} \operatorname{Ric}(W, W) .
$$

So we get the index-free form

$$
\begin{aligned}
H(V, W)= & 2\left(\partial_{t} \operatorname{Ric}\right)(W, W)+2|\operatorname{Ric}(W, \cdot)|^{2}-\nabla_{W} \nabla_{W} R+\frac{1}{t} \operatorname{Ric}(W, W) \\
& -4\left[\left(\nabla_{V} \operatorname{Ric}\right)(W, W)-\left(\nabla_{W} \operatorname{Ric}\right)(V, W)\right]+2\langle R(V, W) W, V\rangle \geq 0 .
\end{aligned}
$$

To get an index-free version of equation ii) in theorem 2.14, we trace this over an orthonormal basis $\left\{W_{i}\right\}$. Note that

$$
\partial_{t} R=\triangle R+2|\operatorname{Ric}|^{2}=\partial_{t}\left(g^{i j} R_{i j}\right)=2|\operatorname{Ric}|^{2}+\sum_{i}\left(\partial_{t} \operatorname{Ric}\right)\left(W_{i}, W_{i}\right),
$$


and hence $\sum_{i}\left(\partial_{t} \operatorname{Ric}\right)\left(W_{i}, W_{i}\right)=\triangle R$. Moreover, we use

$$
\sum_{i}\left(\nabla_{W_{i}} \operatorname{Ric}\right)\left(V, W_{i}\right)=\frac{1}{2}\langle\nabla R, V\rangle,
$$

which follows from the second Bianchi identity. We then get

$$
\begin{aligned}
\sum_{i=1}^{n} H\left(V, W_{i}\right)= & 2 \triangle R+2|\operatorname{Ric}|^{2}-\triangle R+\frac{1}{t} R \\
& -4\left(\langle\nabla R, V\rangle-\sum_{i}\left(\nabla_{W_{i}} \operatorname{Ric}\right)\left(V, W_{i}\right)\right) \\
& +2 \sum_{i}\left\langle R\left(V, W_{i}\right) W_{i}, V\right\rangle \\
= & \partial_{t} R+\frac{1}{t} R-4\left(\langle\nabla R, V\rangle-\frac{1}{2}\langle\nabla R, V\rangle\right)+2 \operatorname{Ric}(V, V) \\
= & \partial_{t} R+\frac{1}{t} R-2\langle\nabla R, V\rangle+2 \operatorname{Ric}(V, V)=H(V) \geq 0 .
\end{aligned}
$$

We can now restate the theorem in a second (index-free) version.

Theorem 2.15 (Harnack estimates for Ricci flow, second version)

Suppose $(M, g)$ is a complete solution to the Ricci flow (1.14) for $t \in[0, T]$ with bounded curvature. Suppose $g$ has a weakly positive curvature operator. Then for any vectors $V, W$ and $t>0$ we have

$$
\begin{gathered}
\text { i) } \begin{aligned}
H(V, W) & :=2\left(\partial_{t} \operatorname{Ric}\right)(W, W)+2|\operatorname{Ric}(W, \cdot)|^{2}-\nabla_{W} \nabla_{W} R+\frac{1}{t} \operatorname{Ric}(W, W) \\
& -4\left[\left(\nabla_{V} \operatorname{Ric}\right)(W, W)-\left(\nabla_{W} \operatorname{Ric}\right)(V, W)\right]-2\langle R(V, W) V, W\rangle \geq 0,
\end{aligned} \text { ii) } H(V):=\partial_{t} R+\frac{1}{t} R-2\langle\nabla R, V\rangle+2 \operatorname{Ric}(V, V) \geq 0 .
\end{gathered}
$$

Remark. The Harnack expressions $H(V, W)$ and $H(V)$ will appear exactly in this form again in chapter four. However, we will not use theorem 2.15 in any essential way there, since surprisingly - the Harnack expressions will cancel out in the final results!

There have been several alternate proofs and geometric interpretations of these Harnack inequalities. We like to point out one of them, which is very interesting: One can define a degenerate metric $\tilde{g}$ on the space-time manifold $\tilde{M}=M \times[0, T]$ in such a way that

$$
\tilde{g}^{i j}= \begin{cases}g^{i j} & \text { if } i, j \geq 1 \\ 0 & \text { if } i=0 \text { or } j=0\end{cases}
$$

where the index 0 denotes the time direction. Associated to this metric is a space-time connection $\tilde{\nabla}$ defined by the Christoffel symbols

$$
\tilde{\Gamma}_{i j}^{k}= \begin{cases}\Gamma_{i j}^{k} & \text { if } i, j, k \geq 1 \\ 0 & \text { if } k=0 \\ -R_{j}^{k} & \text { if } i=0 \text { and } j, k \geq 1 \\ -\frac{1}{2} \nabla^{k} R & \text { if } i=j=0 \text { and } k \geq 1 .\end{cases}
$$


This connection is compatible with the metric in the usual sense that $\tilde{\nabla} \tilde{g}=0$, and it satisfies

$$
\partial_{t} \tilde{\Gamma}_{i j}^{k}=-\tilde{g}^{k \ell}\left(\tilde{\nabla}_{i} \tilde{R}_{j \ell}+\tilde{\nabla}_{j} \tilde{R}_{i \ell}-\tilde{\nabla}_{\ell} \tilde{R}_{i j}\right),
$$

which is formally identical to the equation satisfied by the Levi-Cività connection $\nabla$ of the metric $g$ evolving under the Ricci flow, cf. (1.12).

One can then show that the Riemannian curvature tensor for this space-time connection is exactly Hamilton's matrix Harnack expression for the Ricci flow. Similarly, the Ricci curvature tensor of the space-time connection is Hamilton's trace Harnack expression.

The theorem hence says that weakly positive curvature operator on $M$ implies weakly positive Riemannian curvature on the space-time manifold $\tilde{M}$, and the same holds for the Ricci curvature.

This geometric approach to the Harnack inequalities was found by Bennett Chow and SunChin Chu. The details can be found in their papers [5] and [6].

Remark. With this argument in mind, if the Riemannian or Ricci curvature appear during certain computations for a static manifold, one might not be surprised too much to find these Harnack expressions when one makes the corresponding computation for an evolving manifold, since they can be interpreted as the curvature tensors for the space-time manifold with the above natural space-time connection. We will see in chapter four, when we compute the variation of Perelman's $\mathcal{L}$-distance functional with respect to space-time paths, that this actually really happens!

\section{Entropy formulas}

This chapter provides an exposition of integral quantities that are monotone non-increasing or non-decreasing in time under Ricci flow. We will first present entropy formulas for the heat equation on a static manifold (cf. [25]) and then derive the analogs of these results for steady, shrinking and expanding Ricci solitons. We will also present local versions of these formulas.

Perelman showed in the first section of [28] that the gradient flow of his entropy functional $\mathcal{F}$ is the Ricci flow modified with a family of diffeomorphisms, compare with proposition 1.7. We like to point out an additional motivation here, where the connection to the static case and to shrinking and expanding solitons can be seen even better. This idea is the connection between Perelman's functional and Nash entropy. This was first shown to the author by Tom Ilmanen, [20].

With Nash's entropy we will also be able to show a nice connection between the entropy formulas and the original Li-Yau Harnack inequality from the last chapter. We found most of these ideas in Lei Ni's paper [26]. We will also see an application for the Li-Yau Harnack estimate, namely we will prove that the Li-Yau inequality holds as an equality for the heat kernel for some $t_{0}>0$, if and only if the manifold is isometric to the Euclidean space $\mathbb{R}^{n}$. Similar results will be shown for Ni's entropy formulas for the heat equation on a static manifold. 


\subsection{The static case, part I}

On any closed manifold the heat equation $\partial_{t} u=\Delta u$ may be regarded as the $L^{2}$-gradient flow of the Dirichlet functional $D(u):=\int_{M} \frac{1}{2}|\nabla u|^{2} d V$. We compute

$$
\partial_{t} D=\partial_{t} \int_{M} \frac{1}{2}|\nabla u|^{2} d V=\int_{M}-\triangle u \partial_{t} u d V=\int_{M}-(\triangle u)^{2} d V \leq 0,
$$

so the Dirichlet functional is a monotone quantity for the linear heat equation.

Another monotone quantity is the Nash entropy $N(u)=\int_{M} u \log u d V$. Let $u$ be a positive solution of the heat equation $\square u=0$. A direct computation shows

$$
\begin{aligned}
\partial_{t} N & =\int_{M}\left(\partial_{t} u \cdot \log u+u \frac{1}{u} \partial_{t} u\right) d V=\int_{M} \Delta u \log u+\triangle u d V \\
& =\int_{M} \triangle u \log u d V=\int_{M}-\frac{|\nabla u|^{2}}{u} d V \\
& =\int_{M}-|\nabla f|^{2} e^{-f} d V \leq 0,
\end{aligned}
$$

where $f=-\log u$. So the Nash entropy of a solution of the heat equation on a static manifold is monotone decreasing. To compute the second derivative, we use the following lemma, which can also be used to simplify other computations below.

\section{Lemma 3.1}

Let $u$ be a positive solution of the heat equation $\square u=0$. Define $f=-\log u$ and $w=$ $2 \triangle f-|\nabla f|^{2}$. Then

$$
\left(\partial_{t}-\triangle\right) w=-2\left(|\operatorname{Hess}(f)|^{2}+\operatorname{Ric}(\nabla f, \nabla f)\right)-2\langle\nabla w, \nabla f\rangle .
$$

Proof. With $\left(\partial_{t}-\triangle\right) f=-|\nabla f|^{2}$ we find $w=2 \partial_{t} f+|\nabla f|^{2}=\partial_{t} f+\triangle f$. In the proof of lemma 2.4 we showed that

$$
\triangle|\nabla f|^{2}=2\langle\nabla \triangle f, \nabla f\rangle+2 \operatorname{Ric}(\nabla f, \nabla f)+2|\operatorname{Hess}(f)|^{2},
$$

thus a direct computation gives

$$
\begin{aligned}
\left(\partial_{t}-\triangle\right) w & =2 \partial_{t}\left(\partial_{t} f\right)-2 \triangle\left(\partial_{t} f\right)+\partial_{t}|\nabla f|^{2}-\triangle|\nabla f|^{2} \\
& =2 \partial_{t}\left(\partial_{t} f-\triangle f\right)+\partial_{t}|\nabla f|^{2}-\triangle|\nabla f|^{2} \\
& =-\partial_{t}|\nabla f|^{2}-\triangle|\nabla f|^{2} \\
& =-2\left\langle\nabla\left(\partial_{t}+\triangle f\right), \nabla f\right\rangle-2 \operatorname{Ric}(\nabla f, \nabla f)-2|\operatorname{Hess}(f)|^{2} .
\end{aligned}
$$

This is precisely the assertion of the lemma.

With $\nabla u=-u \nabla f$ and $\square u=0$, we get

$$
\begin{aligned}
\left(\partial_{t}-\triangle\right)(w u)= & -2 u\left(|\operatorname{Hess}(f)|^{2}+\operatorname{Ric}(\nabla f, \nabla f)\right) \\
& -2 u\langle\nabla w, \nabla f\rangle-2\langle\nabla w, \nabla u\rangle+w\left(\partial_{t}-\triangle\right) u \\
= & -2 u\left(|\operatorname{Hess}(f)|^{2}+\operatorname{Ric}(\nabla f, \nabla f)\right) .
\end{aligned}
$$


Note that we can write

$$
w u=\left(2 \triangle f-|\nabla f|^{2}\right) e^{-f}=|\nabla f|^{2} e^{-f}-2 \triangle\left(e^{-f}\right),
$$

so for the second derivative of the Nash entropy we find the following formula for $u$ which satisfy the heat equation,

$$
\begin{aligned}
\partial_{t}^{2} N(u) & =\partial_{t} \int_{M}-|\nabla f|^{2} e^{-f} d V \\
& =\int_{M} 2 u\left(|\operatorname{Hess}(f)|^{2}+\operatorname{Ric}(\nabla f, \nabla f)\right) d V,
\end{aligned}
$$

i.e. the derivative of the Nash entropy is monotone non-decreasing under the heat equation if Ric $\geq 0$ on $M$. In other words, the Nash entropy is convex in this case. We will now see an analog to this equation for the Ricci flow. In this way we obtain what is known as Perelman's entropy formula for steady solitons.

\subsection{Entropy for steady Ricci solitons}

Recall, that for a steady soliton with potential $f$, the function $u=e^{-f}$ solves the adjoint heat equation under Ricci flow, see chapter one. So let now $g_{i j}$ be a solution of the Ricci flow (1.14) and $u=e^{-f}$ a (positive) solution of $\square^{*} u=0$ and define the Nash entropy formally as above, $N(u)=\int_{M} u \log u d V$. Differentiating gives

$$
\begin{aligned}
\partial_{t} N & =\int_{M}\left(\partial_{t} u \cdot \log u d V+u \frac{1}{u} \partial_{t} u d V+u \log u \partial_{t} d V\right) \\
& =\int_{M}\left(\left(\partial_{t}-R\right) u \log u+\partial_{t} u\right) d V \\
& =\int_{M}(-\triangle u \log u+(R-\triangle) u) d V .
\end{aligned}
$$

Because the integral of $\triangle u$ vanishes on the closed manifold $M$ and partial integration yields $\int_{M}-\triangle u \log u d V=\int_{M} \frac{|\nabla u|^{2}}{u} d V$, we have

$$
\partial_{t} N=\int_{M}\left(\frac{|\nabla u|^{2}}{u}+R u\right) d V=\int_{M}\left(|\nabla f|^{2}+R\right) e^{-f} d V
$$

Converse to the static case, equation (3.1), the derivative here does not have a sign, unless we assume $R \geq 0$ on $M$. However, we will see below, proposition 3.4, that the second derivative $\partial_{t}^{2} N(u)$ is non-negative (i.e. $N(u)$ is convex) without a-priori assumptions on the curvature, while we needed Ric $\geq 0$ in the static case to get such a result. Hence (3.3) is a monotone quantity along the Ricci flow and may be interpreted as an entropy functional.

Definition 3.2 (Perelman's $\mathcal{F}$-entropy, section one of [28])

We define the $\mathcal{F}$-functional for a Riemannian metric $g_{i j}$ and a function $f \in C^{\infty}(M)$ by

$$
\mathcal{F}\left(g_{i j}, f\right)=\int_{M}\left(|\nabla f|^{2}+R\right) e^{-f} d V
$$

restricted to $f$ that satisfy $\int_{M} e^{-f} d V \equiv 1$. 
Note that for a solution $u=e^{-f}$ of the adjoint heat equation $\square^{*} u=0$ and a solution $g(t)$ of the Ricci flow equation (1.14), $\int_{M} u d V$ is preserved and hence the assumption $\int_{M} e^{-f} d V \equiv 1$ is satisfied if initially true. Moreover, for this setting, $\mathcal{F}(g, f)=\partial_{t} N(u)$ is simply the first derivative of the Nash entropy.

We may interpret $\mathcal{F}$ as a combination of the entropy functional $\int_{M}|\nabla f|^{2} e^{-f} d V$ from the static case, equation (3.2), with the Einstein-Hilbert functional defined in chapter one. We are now interested in its gradient flow. Perelman noticed that the first variation of his functional is

$$
\delta_{h} \mathcal{F}=\int_{M}-h^{i j}\left(R_{i j}+\nabla_{i} \nabla_{j} f\right) e^{-f} d V
$$

if the measure $d m=e^{-f} d V$ is pointwise kept fixed. This follows from the following lemma.

Lemma 3.3 (Perelman, section 1.1 of [28])

Let $\partial_{s} g_{i j}=h_{i j}$ and $\partial_{s} f=\ell$ be variations of the Riemannian metric $g_{i j}$ and the function $f$. Then the first variation of $\mathcal{F}\left(g_{i j}, f\right)$ is

$$
\partial_{s} \mathcal{F}=\int_{M}\left[-h^{i j}\left(R_{i j}+\nabla_{i} \nabla_{j} f\right)+\left(\frac{1}{2} \operatorname{tr}_{g} h-\ell\right)\left(2 \triangle f-|\nabla f|^{2}+R\right)\right] e^{-f} d V .
$$

Proof. We need the evolution equations

$$
\begin{aligned}
\partial_{s} R & =-h^{i k} R_{i k}-\triangle\left(\operatorname{tr}_{g} h\right)+\operatorname{div}^{2} h, \\
\partial_{s} d V & =\frac{1}{2} \operatorname{tr}_{g} h d V
\end{aligned}
$$

from chapter one, as well as

$$
\begin{aligned}
\partial_{s}|\nabla f|^{2} & =\delta\langle\nabla f, \nabla f\rangle_{g}=2\langle\nabla f, \nabla \ell\rangle-h^{i k} \nabla_{i} f \nabla_{k} f, \\
\partial_{s} e^{-f} & =-\ell e^{-f} .
\end{aligned}
$$

By simply inserting these four expressions, we find

$$
\begin{aligned}
\partial_{s} \mathcal{F}=\int_{M}\left[\partial_{s}\right. & R e^{-f} d V+\partial_{s}|\nabla f|^{2} e^{-f} d V \\
& \left.+\left(R+|\nabla f|^{2}\right)\left(\partial_{s} e^{-f} d V+e^{-f} \partial_{s} d V\right)\right] \\
=\int_{M}[- & h^{i k} R_{i k}-\triangle\left(\operatorname{tr}_{g} h\right)+\operatorname{div}^{2} h-h^{i k} \nabla_{i} f \nabla_{k} f \\
& \left.+2\langle\nabla f, \nabla \ell\rangle_{g}+\left(R+|\nabla f|^{2}\right)\left(\frac{1}{2} \operatorname{tr}_{g} h-\ell\right)\right] e^{-f} d V .
\end{aligned}
$$

We now rewrite some of these terms using partial integration. With $\operatorname{div}^{2} h=\nabla_{i} \nabla_{k} h^{i k}$ and $\nabla_{i} \nabla_{k} e^{-f}=\left(\nabla_{i} f \nabla_{k} f-\nabla_{i} \nabla_{k} f\right) e^{-f}$, one finds

$$
\int_{M}\left(\operatorname{div}^{2} h-h^{i k} \nabla_{i} f \nabla_{k} f\right) e^{-f} d V=\int_{M}-h^{i k} \nabla_{i} \nabla_{k} f e^{-f} d V .
$$

Moreover, $-\triangle e^{-f}=\left(\triangle f-|\nabla f|^{2}\right) e^{-f}$ yields

$$
\int_{M}\left(-\triangle\left(\operatorname{tr}_{g} h\right)+|\nabla f|^{2} \cdot \frac{1}{2} \operatorname{tr}_{g} h\right) e^{-f} d V=\int_{M}\left(2 \triangle f-|\nabla f|^{2}\right) \frac{1}{2} \operatorname{tr}_{g} h e^{-f} d V .
$$


Finally, one computes

$$
\begin{aligned}
\int_{M}\left(2\langle\nabla f, \nabla \ell\rangle-|\nabla f|^{2} \ell\right) e^{-f} d V & =-\int_{M}\left(2 \operatorname{div}\left(\nabla f e^{-f}\right)+|\nabla f|^{2} e^{-f}\right) \ell d V \\
& =-\int_{M}\left(2 \triangle f-|\nabla f|^{2}\right) \ell e^{-f} d V
\end{aligned}
$$

where we used again $\operatorname{div}\left(\nabla f e^{-f}\right)=\left(\triangle f-|\nabla f|^{2}\right) e^{-f}$. The lemma now follows by inserting the equations (3.6)-(3.8) into (3.5).

Remark. If $d m=e^{-f} d V$ is pointwise kept fixed, then

$$
\partial_{s}\left(e^{-f} d V\right)=\left(\frac{1}{2} \operatorname{tr}_{g} h-\ell\right) e^{-f} d V=0
$$

and equation (3.4) follows directly from lemma 3.3.

We hence get the following system of evolution equations for the $L^{2}$-gradient flow of Perelman's F-functional

$$
\begin{cases}\partial_{t} g_{i j} & =-2\left(R_{i j}+\nabla_{i} \nabla_{j} f\right), \\ \partial_{t} f & =\frac{1}{2} \operatorname{tr}_{g}\left(\partial_{t} g_{i j}\right)=-\triangle f-R .\end{cases}
$$

We now look at the pull-back of this system with the family of diffeomorphisms with generating vector field $X=\nabla f$. Let $\phi: M \rightarrow M$ be generated by $\nabla f$, i.e.

$$
\partial_{t} \phi\left(t, t_{0}\right)=X(t) \circ \phi\left(t, t_{0}\right), \quad \phi\left(t_{0}, t_{0}\right)=\mathrm{id}_{M},
$$

and write $\phi(t)$ for $\phi(t, 0)$. Moreover, let $\tilde{g}:=\phi^{*} g, \tilde{f}:=\phi^{*} f$ be the pull-backs of the metric $g$ and the function $f$ on $M$. Then

$$
\begin{aligned}
\left.\partial_{t} \tilde{g}\right|_{t=t_{0}} & =\left.\partial_{t}\left(\phi^{*} g\right)\right|_{t=t_{0}}=\left.\partial_{\varepsilon}\right|_{\varepsilon=0}\left(\phi\left(t_{0}+\varepsilon\right)^{*} g\right) \\
& =\left.\partial_{\varepsilon}\right|_{\varepsilon=0}\left(\phi\left(t_{0}\right)^{*} \phi\left(t_{0}+\varepsilon, t_{0}\right)^{*} g\right)=\phi\left(t_{0}\right)^{*}\left(L_{X\left(t_{0}\right)} g+\left.\partial_{t} g\right|_{t=t_{0}}\right) \\
& =\phi\left(t_{0}\right)^{*}\left(2 \nabla_{i} \nabla_{j} f\left(t_{0}\right)-2\left(R_{i j}\left(g\left(t_{0}\right)\right)+\nabla_{i} \nabla_{j} f\left(t_{0}\right)\right)\right) \\
& =-2 \phi\left(t_{0}\right)^{*} \operatorname{Ric}\left(g\left(t_{0}\right)\right)=-2 \operatorname{Ric}\left(\tilde{g}\left(t_{0}\right)\right),
\end{aligned}
$$

as well as

$$
\begin{aligned}
\partial_{t} \tilde{f} & =\partial_{t}\left(\phi^{*} f\right)=\partial_{t} f \circ \phi+d f \circ \partial_{t} \phi(t) \\
& =\phi^{*} \partial_{t} f+d f \circ \nabla f \circ \phi \\
& =\phi^{*}(-\triangle f-R)+\phi^{*}|\nabla f|^{2} . \\
& =-\triangle \tilde{f}+|\nabla \tilde{f}|^{2}-R(\tilde{g})
\end{aligned}
$$

Hence the pull-backs satisfy

$$
\begin{cases}\partial_{t} \tilde{g}_{i j} & =-2 R_{i j} \\ \partial_{t} \tilde{f} & =-\triangle \tilde{f}+|\nabla \tilde{f}|^{2}-R\end{cases}
$$

where $\triangle,|\cdot|$, Ric and $R$ depend on the metric $\tilde{g}$. Note that the second equation is equivalent to $\square^{*} e^{-\tilde{f}}=0$. Thus the system $\left(g_{i j}, u\right)$ where $g_{i j}$ solves the Ricci flow and $u>0$ solves the adjoint heat equation may be regarded as the gradient flow system of $\mathcal{F}$ modulo the family of diffeomorphisms generated by $\nabla f$. This nice result neutralizes the bad result from proposition 1.7.

With (3.4) and (3.9), we get the convexity of $N(u)$ claimed above. 
Proposition 3.4 (Perelman, section 1.1 of [28])

Let $g_{i j}$ be a solution of the Ricci flow $\partial_{t} g=-2$ Ric and let $u=e^{-f}$ be a positive solution of the adjoint heat equation. Then the $\mathcal{F}$-functional satisfies

$$
\partial_{t} \mathcal{F}=\partial_{t}^{2} N=\int_{M} 2 u|\operatorname{Hess}(f)+\operatorname{Ric}|^{2} d V \geq 0,
$$

hence $\mathcal{F}$ is monotone increasing in time and constant exactly on steady Ricci solitons with potential $f$ which satisfy $\nabla_{i} \nabla_{j} f+R_{i j}=0$. Moreover, $N(u)$ is convex and strictly convex away from steady solitons.

Similar results for shrinking and expanding solitons will be explained below. Especially the shrinking soliton entropy turns out to be very important. In order to find similar functionals to $\mathcal{F}$ which are constant on shrinkers (or expanders), we have to look for modifications that include an explicit factor $t$ to control the shrinking (or expanding) process. We first do this for the static case.

\subsection{The static case, part II}

We return again to the heat equation $\square u=0$ on a static closed manifold $M$. In the following, we will always write a positive solution $u$ of $\square u=0$ in the form $u=(4 \pi t)^{-n / 2} e^{-f}$.

We look at a modification of the functional $\int_{M}|\nabla f|^{2} e^{-f} d V$ from equation (3.2). This is taken from Lei Ni's papers [25] and [26]. Define the entropy for the heat equation on a static manifold by

$$
\mathcal{W}(u, t):=\int_{M}\left(t|\nabla f|^{2}+f-n\right) u d V
$$

restricted to $(u, t)$ which satisfy $\int_{M} u d V \equiv 1$. Note that $\int_{M} u d V$ is preserved if $\square u=0$, because $\partial_{t} \int_{M} u d V=\int_{M} \partial_{t} u d V=\int_{M} \Delta u d V=0$. We get a similar result to the one in equation (3.2). The following result goes back to Lei Ni.

Proposition 3.5 (entropy for heat equation)

For any closed $M$, a positive solution $u=(4 \pi t)^{-n / 2} e^{-f}$ of the heat equation $\square u=0$ on $M$, and $\mathcal{W}(u, t)$ defined as above, we have

$$
\frac{d}{d t} \mathcal{W}(u, t)=-\int_{M} 2 u t\left(\left|\operatorname{Hess}(f)-\frac{g}{2 t}\right|^{2}+\operatorname{Ric}(\nabla f, \nabla f)\right) d V .
$$

In particular, if $M$ has non-negative Ricci curvature, $\mathcal{W}(u, t)$ is monotone decreasing along the heat equation.

Proof. We use lemma 3.1 to simplify the computation. Define

$$
W(u, t)=t\left(2 \triangle f-|\nabla f|^{2}\right)+f-n .
$$

With $u=e^{-\tilde{f}}=(4 \pi t)^{-n / 2} e^{-f}$, we get $f=\tilde{f}-\frac{n}{2} \log (4 \pi t)$, and hence $\nabla \tilde{f}=\nabla f, \triangle \tilde{f}=\triangle f$ and $\operatorname{Hess}(\tilde{f})=\operatorname{Hess}(f)$. We can thus rewrite $W$ as

$$
W(u, t)=t w+\tilde{f}-\frac{n}{2} \log (4 \pi t)-n,
$$


where $w=2 \triangle \tilde{f}-|\nabla \tilde{f}|^{2}=2 \triangle f-|\nabla f|^{2}$ is defined as in lemma 3.1. We compute

$$
\begin{aligned}
\left(\partial_{t}-\triangle\right) W= & t\left(\partial_{t}-\triangle\right) w+w+\left(\partial_{t}-\triangle\right) \tilde{f}-\frac{n}{2 t} \\
= & -2 t\left(|\operatorname{Hess}(f)|^{2}+\operatorname{Ric}(\nabla f, \nabla f)\right)-2 t\langle\nabla w, \nabla f\rangle \\
& +\left(2 \partial_{t} \tilde{f}+|\nabla f|^{2}\right)-|\nabla f|^{2}-\frac{n}{2 t} \\
= & -2 t\left(|\operatorname{Hess}(f)|^{2}+\operatorname{Ric}(\nabla f, \nabla f)\right) \\
& -2\langle\nabla(W-\tilde{f}), \nabla f\rangle+2 \partial_{t} \tilde{f}-\frac{n}{2 t} \\
= & -2 t\left(|\operatorname{Hess}(f)|^{2}+\operatorname{Ric}(\nabla f, \nabla f)\right)+2 \triangle f-\frac{n}{2 t}-2\langle\nabla W, \nabla f\rangle \\
= & -2 t\left(\left|\operatorname{Hess}(f)-\frac{g}{2 t}\right|^{2}+\operatorname{Ric}(\nabla f, \nabla f)\right)-2\langle\nabla W, \nabla f\rangle .
\end{aligned}
$$

Finally with $u \nabla f=-\nabla u$ and $\square u=0$, we obtain

$$
\begin{aligned}
\left(\partial_{t}-\triangle\right)(W u)= & -2 u t\left(\left|\operatorname{Hess}(f)-\frac{g}{2 t}\right|^{2}+\operatorname{Ric}(\nabla f, \nabla f)\right) \\
& -2\langle\nabla W, \nabla f\rangle u-2\langle\nabla W, \nabla u\rangle+W\left(\partial_{t}-\triangle\right) u \\
= & -2 u t\left(\left|\operatorname{Hess}(f)-\frac{g}{2 t}\right|^{2}+\operatorname{Ric}(\nabla f, \nabla f)\right) .
\end{aligned}
$$

Note that

$$
\begin{aligned}
\int_{M} W u d V & =\int_{M}\left(t\left(2 \triangle f-|\nabla f|^{2}\right)+f-n\right) u d V \\
& =\int_{M}\left(t|\nabla f|^{2}+f-n\right) u d V+2 \int_{M}\left(\triangle f-|\nabla f|^{2}\right) u d V \\
& =\mathcal{W}(u, t),
\end{aligned}
$$

since in view of $\left(\triangle f-|\nabla f|^{2}\right) u=-\triangle u$ the second integral on the second line vanishes on closed $M$. This proves the proposition.

There is a good reason why we introduced the expression $W(u, t)$ in the proof, equation (3.12). In fact we may interpret $W u$ as the local version of the entropy $\mathcal{W}(u, t)=\int_{M} W u d V$. Note that a function $W^{\prime}(u, t)$ that differs from $W(u, t)$ by a divergence term will also satisfy $\mathcal{W}(u, t)=\int_{M} W u d V$, so at first glance there are many different local versions of $\mathcal{W}(u, t)$, but since we want the local entropy to satisfy a pointwise inequality if $u$ is the heat kernel, we choose $W(u, t)$ as in (3.12).

Proposition 3.6 (local entropy, theorem 1.2 of [25])

Let $u=(4 \pi t)^{-n / 2} e^{-f}$ be the positive heat kernel on $M$, i.e. $u$ tends to a $\delta$-function as $t \rightarrow 0$ and satisfies $\int_{M} u d V \equiv 1$. Let $M$ be closed with non-negative Ricci curvature and let $W(u, t)$ be as in (3.12). Then for $t>0$ we have

$$
W(u, t)=t\left(2 \triangle f-|\nabla f|^{2}\right)+f-n \leq 0 .
$$


Proof. For any $t_{0}>0$, let $h$ be any positive smooth function. Then define $h(\cdot, t)$ for $t \leq t_{0}$ such that $h$ satisfies the backwards heat equation $\partial_{t} h=-\triangle h$. A direct computation shows that

$$
\begin{aligned}
\partial_{t} \int_{M} h W u d V & =\int_{M}\left(\partial_{t} h(W u)+h \partial_{t}(W u)\right) d V \\
& =\int_{M}(\underbrace{\left(\partial_{t}+\triangle\right) h}_{=0}(W u)+h \underbrace{\left(\partial_{t}-\triangle\right)(W u)}_{\leq 0}) d V \leq 0 .
\end{aligned}
$$

If $u$ tends to a $\delta$-function (at a point $p \in M$ ) for $t \rightarrow 0$, then $f$ satisfies

$$
f(x, t) \leq \frac{d^{2}(p, x)}{4 t}
$$

a fact which we will prove in the last chapter, corollary 4.3. This yields

$$
\lim _{t \rightarrow 0} \int_{M} f h u d V \leq \limsup _{t \rightarrow 0} \int_{M} \frac{d^{2}(p, x)}{4 t} h u d V=\frac{n}{2} h(p, 0),
$$

where the last equality is taken from [27], section 3. In [27], $u$ is actually the adjoint heat kernel on an evolving manifold $M$ and $h$ satisfies the forward heat equation, but (3.15)also holds in our case. Note that the (original) Li-Yau Harnack inequality holds since Ric $\geq 0$ and that for $u=(4 \pi t)^{-n / 2} e^{-f}$ it can be written in the form $H(u, t)=-t \triangle f+\frac{n}{2} \geq 0$. So by adding $H(u, t) h u \geq 0$ to the integrand, we get

$$
\begin{aligned}
\lim _{t \rightarrow 0} \int_{M} h W u d V & =\lim _{t \rightarrow 0} \int_{M}\left[t\left(2 \triangle f-|\nabla f|^{2}\right)+f-n\right] h u d V \\
& \leq \lim _{t \rightarrow 0} \int_{M}\left[t\left(\triangle f-|\nabla f|^{2}\right)+f-\frac{n}{2}\right] h u d V \\
& =\lim _{t \rightarrow 0} \int_{M}\left[\left(f-\frac{n}{2}\right) h u-t u \triangle h\right] d V \\
& =\lim _{t \rightarrow 0} \int_{M}\left(f-\frac{n}{2}\right) h u \leq 0,
\end{aligned}
$$

where we used (3.15) in the very last line. Thus $\int_{M} h W u d V \leq 0$ for any $t_{0}$ and any positive function $h$. This implies $W u \leq 0$ and because $u$ is positive, we get $W \leq 0$ as claimed.

Note that $W$ vanishes identically for the heat kernel $\bar{u}=(4 \pi t)^{-n / 2} e^{-|x|^{2} / 4 t}$ on the Euclidean space $\mathbb{R}^{n}$ as an easy computation shows. Actually this is the only case where $W$ can vanish for a strictly positive time $t_{0}>0$ :

Proposition 3.7 (Lei Ni)

Let $M$ be a closed manifold with Ric $\geq 0$ and let $u=(4 \pi t)^{-n / 2} e^{-f}$ be the heat kernel on $M$ as in proposition 3.6. Then $\mathcal{W}\left(u, t_{0}\right) \geq 0$ for some $t_{0}>0$ if and only if $M$ is isometric to $\mathbb{R}^{n}$.

Proof. We only give a heuristic argument without analyzing the error terms when we write that $f$ is close to $\bar{f}$ for the heat kernel. A rigorous proof (for example with parabolic rescalings) is left to the reader. 
Proposition 3.6 implies $\mathcal{W}(u, t) \leq 0$. Moreover, proposition 3.5 shows that $\frac{d}{d t} \mathcal{W}(u, t) \leq 0$, so we must have $\mathcal{W}(u, t) \equiv 0$ for $0 \leq t \leq t_{0}$. But - again with proposition 3.5 - this implies that $|\operatorname{Hess}(f)-g / 2 t|^{2}=0$, i.e. $\nabla_{i} \nabla_{j} f-g_{i j} / 2 t=0$. Taking the trace, we find

$$
2 t \triangle f-n=0 .
$$

Because $f(x, t) \approx \bar{f}(x, t)=\frac{d^{2}(p, x)}{4 t}$ for $t$ small and $p$ being the point where the $\delta$-function forms, we have $\lim _{t \rightarrow 0} 4 t f=d^{2}(p, x)$. Hence (3.16) implies that $\triangle d^{2}(p, x)=2 n$. So we get for the area $A_{p}(r)$ of $\partial B_{p}(r)$ and the volume $V_{p}(r)$ of the ball $B_{p}(r)$ the following quotient:

$$
\frac{A_{p}(r)}{V_{p}(r)}=\frac{n}{r}
$$

This shows that $V_{p}(r)$ is exactly the same as the volume function for Euclidean balls. One can then use the volume comparison theorem with Ric $\geq 0$. The equality case implies that $M$ is isometric to $\mathbb{R}^{n}$. The details are left to the reader.

Remark. This argument shows that the Li-Yau Harnack inequality, which is equivalent to $2 t \triangle f-n \leq 0$ for $u=(4 \pi t)^{-n / 2} e^{-f}$, becomes an equality (i.e. the above equation (3.16) holds), if and only if the manifold $M$ (with Ric $\geq 0$ ) is isometric to $\mathbb{R}^{n}$ and $u$ is the heat kernel.

After this technical part, we want to give another motivation for the choice of the entropy functional $\mathcal{W}(u, t)$ which connects this section with the previous ones. Lei Ni showed an interesting relation between Li-Yau's gradient estimate, the (local) entropy formula and Nash's entropy. Let again $u>0$ solve $\square u=0$ and $\int_{M} u d V=1$. Let $N(u)=\int_{M} u \log u d V$ be the Nash entropy as before, and set

$$
\tilde{N}(u, t)=N(u)+\frac{n}{2} \log (4 \pi t)+\frac{n}{2} .
$$

Note that we can write this as

$$
\tilde{N}(u, t)=\int_{M}\left(\log \left((4 \pi t)^{n / 2} u\right)+\frac{n}{2}\right) u d V=\int_{M}\left(-f+\frac{n}{2}\right) u d V .
$$

We get the derivative

$$
\tilde{F}(u, t):=\frac{d}{d t} \tilde{N}(u, t)=\int_{M} \triangle u \cdot \log u d V+\frac{n}{2 t}=\int_{M}\left(\triangle(\log u)+\frac{n}{2 t}\right) u d V .
$$

Using

$$
\int_{M} t \triangle(\log u) u d V=-\int_{M} t \nabla(\log u) \nabla u d V=-\int_{M} t \frac{|\nabla u|^{2}}{u} d V
$$

we can write the entropy functional as

$$
\begin{aligned}
\mathcal{W}(u, t) & =\int_{M}\left(t|\nabla f|^{2}-\frac{n}{2}\right) u d V+\int_{M}\left(f-\frac{n}{2}\right) u d V \\
& =-t \tilde{F}(u, t)-\tilde{N}(u, t)=-\frac{d}{d t}(t \tilde{N}(u, t)) .
\end{aligned}
$$

This shows that the entropy is again the derivative of the modified Nash entropy $t \tilde{N}(u, t)$. Moreover, there is a surprising connection to the Li-Yau result: the integrand of $\tilde{F}(u, t)$ is exactly their Harnack expression

$$
\left(\triangle(\log u)+\frac{n}{2 t}\right) u=\left(-\triangle f+\frac{n}{2 t}\right) u=\triangle u-\frac{|\nabla u|^{2}}{u}+\frac{n u}{2 t}=H(u, t) .
$$


Hence an immediate consequence is that $\tilde{N}(u, t)$ is monotone increasing when $M$ has nonnegative Ricci curvature, because the Harnack estimate holds in this case, which implies $\tilde{F}(u, t) \geq 0$. Moreover, one can see that $\lim _{t \rightarrow 0} \tilde{N}(u, t)=0$ for the heat kernel, and hence, if $M$ has non-negative Ricci curvature, we get $\tilde{N}(u, t) \geq 0$, too. We are thus led to the following stronger version of proposition 3.7.

Theorem 3.8 (entropy for heat kernel)

Let $\mathcal{W}(u, t), \tilde{N}(u, t)$ and $\tilde{F}(u, t)$ be as above, and let $H(u, t)$ be the Li-Yau Harnack expression. Under the hypotheses of proposition 3.5 for $M$ and $u$, the following are equivalent:

i) $\exists t_{0}>0$ such that $\mathcal{W}\left(u, t_{0}\right) \geq 0$,

ii) $\exists t_{0}>0$ such that $\tilde{N}\left(u, t_{0}\right) \leq 0$,

iii) $\exists t_{0}>0$ such that $\tilde{F}\left(u, t_{0}\right) \leq 0$,

iv) $\exists t_{0}>0$ such that $H\left(u, t_{0}\right) \leq 0$,

v) $M$ is isometric to the Euclidean space $\mathbb{R}^{n}$,

vi) $\forall t>0$ we have $\mathcal{W}(u, t)=\tilde{N}(u, t)=\tilde{F}(u, t)=H(u, t)=0$.

Proof. i) $\Rightarrow$ ii), iii): This is obvious, since $\mathcal{W}(u, t)=-t \tilde{F}(u, t)-\tilde{N}(u, t)$ and both $\tilde{F}(u, t)$ and $\tilde{N}(u, t)$ are non-negative.

ii) $\Rightarrow$ iii): $\tilde{N}(u, t)$ is monotone increasing and non-negative. Thus $\tilde{N}\left(u, t_{0}\right)=0$ implies $\tilde{N}(u, t)=0$ for all $t \in\left[0, t_{0}\right]$. Hence $\tilde{F}(u, t)=\frac{d}{d t} \tilde{N}(u, t)=0$ for all $t \in\left[0, t_{0}\right)$.

iii) $\Rightarrow$ iv): $\tilde{F}\left(u, t_{0}\right)=\int_{M} H\left(u, t_{0}\right) d V=0$ and the Li-Yau Harnack estimate states that $H\left(u, t_{0}\right) \geq 0$. Hence we must have $H\left(u, t_{0}\right) \equiv 0$ on $M$.

iv) $\Rightarrow \mathrm{v}$ ): This follows from the remark after proposition 3.7.

v) $\Rightarrow$ vi): On $\mathbb{R}^{n}$ (with $\left.f(x, t)=\bar{f}(x, t)=|x|^{2} / 4 t\right)$ we immediately get vi), and thus it will also hold for $M$ isometric to $\mathbb{R}^{n}$.

vi) $\Rightarrow$ i): This is trivial.

The remarkable similarity between the Li-Yau Harnack inequality,

$$
H(u, t)=t(2 \triangle f)-n \leq 0,
$$

and the local entropy formula for the heat kernel,

$$
W(u, t)=t\left(2 \triangle f-|\nabla f|^{2}\right)+f-n \leq 0,
$$

implies that one can use similar technics to the ones we used in chapter two to find more results about the local entropy $W$. For example, $W$ also satisfies a non-linear heat equation similar to (2.18) and hence stays non-positive, if this is initially true. But, different from the Li-Yau expression, this does not have to be initially so, except for the heat kernel!

Finally we try to integrate the pointwise gradient inequality (3.19) along a path, similarly to corollary 2.7, where we integrated Li-Yau's Harnack expression. To do that, we first have to write it in a different form. 
Proposition 3.9 (differential Harnack for heat kernel on a static manifold)

Let $u=(4 \pi t)^{-n / 2} e^{-f}$ be the heat kernel on a closed manifold $M$ with non-negative Ricci curvature and let $\gamma(t)$ ba a smooth curve in $M$. Then for $t>0$, we have the estimate

$$
\frac{d}{d t} f(\gamma(t), t) \leq \frac{1}{2}|\dot{\gamma}(t)|^{2}-\frac{f(\gamma(t), t)}{2 t} .
$$

Proof. Proposition 3.6 says that (3.19) holds. Multiplying equation (3.19) by $-\frac{1}{2 t}$ yields

$$
-\triangle f+\frac{1}{2}|\nabla f|^{2}+\frac{n-f}{2 t} \geq 0
$$

If we insert the evolution equation $\square u=0$ or, in terms of $f, \triangle f=\partial_{t} f+|\nabla f|^{2}+\frac{n}{2 t}$ into this inequality, we get

$$
-\partial_{t} f-\frac{1}{2}|\nabla f|^{2}-\frac{f}{2 t} \geq 0 .
$$

The lemma now follows with Young's inequality,

$$
\frac{d}{d t} f(\gamma(t), t)=\partial_{t} f+\langle\nabla f, \dot{\gamma}\rangle \leq \partial_{t} f+\frac{1}{2}|\nabla f|^{2}+\frac{1}{2}|\dot{\gamma}|^{2} \leq \frac{1}{2}|\dot{\gamma}|^{2}-\frac{f}{2 t},
$$

where we added the positive expression from (3.20) in the last step.

To be able to integrate this, we must get rid of the second term on the right hand side. We can do this by multiplication of $f$ by $2 \sqrt{t}$, which yields

$$
\frac{d}{d t}(2 \sqrt{t} f)=2 \sqrt{t} \frac{d}{d t} f+\frac{f}{\sqrt{t}} \leq \sqrt{t}|\dot{\gamma}|^{2} .
$$

This implies

$$
2 \sqrt{T} f(y, T)=\left.2 \sqrt{t} f(\gamma(t), t)\right|_{0} ^{T}=\int_{0}^{T} \frac{d}{d t}(2 \sqrt{t} f) d t \leq \int_{0}^{T} \sqrt{t}|\dot{\gamma}|^{2} d t .
$$

Hence, we get a similar result to corollary 2.7 , namely

Corollary 3.10 (integrated version)

Let $u$ be the heat kernel on a closed manifold $M$ with $\operatorname{Ric} \geq 0$, and let $p \in M$ be the point where the $\delta$-function forms for $t \rightarrow 0$. Then

$$
u(q, T)=(4 \pi T)^{-n / 2} e^{-f(q, T)} \geq(4 \pi T)^{-n / 2} e^{-\ell(q, T)},
$$

where

$$
\ell(q, T):=\inf _{\gamma \in \Gamma}\left\{\frac{1}{2 \sqrt{T}} \int_{0}^{T} \sqrt{t}|\dot{\gamma}|^{2} d t\right\}
$$

with

$$
\Gamma:=\{\gamma:[0, T] \rightarrow M \mid \gamma(0)=p, \gamma(T)=q\}
$$

We call the above-defined distance function $\ell(q, T)$ the reduced distance. 


\subsection{Entropy for shrinking solitons}

Recall that Perelman's steady soliton entropy was the analog of the static case entropy functional $\int_{M}|\nabla f|^{2} e^{-f} d V$ in (3.2). In the same way one finds an entropy functional for shrinking solitons by looking at the analog of the modified static case entropy $\mathcal{W}(f, t)$.

Let $g_{i j}$ be a complete solution to the Ricci flow for $0 \leq t \leq T$. Set $\tau:=(T-t)$ as before for the shrinking case. Let $u=(4 \pi \tau)^{-n / 2} e^{-f}>0$ satisfy the adjoint heat equation $\square^{*} u=\left(-\partial_{t}-\triangle+R\right) u=0$. First note that this is equivalent to $f$ satisfying the equation

$$
\left(\partial_{t}+\triangle\right) f=|\nabla f|^{2}-R+\frac{n}{2 \tau} .
$$

Proof. With $\triangle\left(e^{-f}\right)=\left(-\triangle f+|\nabla f|^{2}\right) e^{-f}$ we get

$$
\begin{aligned}
0 & =\square^{*} u=\left(-\partial_{t}-\triangle+R\right)\left[(4 \pi \tau)^{-n / 2} e^{-f}\right] \\
& =\left[-\partial_{t}(4 \pi \tau)^{-n / 2}\right] e^{-f}+(4 \pi \tau)^{-n / 2}\left(-\partial_{t}-\triangle\right) e^{-f}+R u \\
& =-\frac{n}{2 \tau} u+\left[\partial_{t} f+\triangle f-|\nabla f|^{2}\right] u+R u \\
& =\left[-\frac{n}{2 \tau}+\left(\partial_{t}+\triangle\right) f-|\nabla f|^{2}+R\right] u
\end{aligned}
$$

This proves the claim, because $u$ is positive.

We can now prove the following lemma, which is similar to the static case above, compare with the proof of proposition 3.5.

Lemma 3.11 (Perelman, proposition 9.1 of [28])

Define $v=W u=\left[\tau\left(2 \triangle f-|\nabla f|^{2}+R\right)+f-n\right]$. Then $v$ satisfies

$$
\square^{*} v=-2 \tau\left|\operatorname{Ric}+\operatorname{Hess}(f)-\frac{g}{2 \tau}\right|^{2} u .
$$

Proof. We will show that

$$
\begin{aligned}
u^{-1} \square^{*} v= & -2 \tau\left|R_{i j}+\nabla_{i} \nabla_{j} f-\frac{1}{2 \tau} g_{i j}\right|^{2} \\
= & -2 \tau\left(|\operatorname{Ric}|^{2}+2 R_{i j} \nabla_{i} \nabla_{j} f+|\operatorname{Hess}(f)|^{2}\right) \\
& +2 \tau\left(\frac{1}{\tau} g_{i j} R_{i j}+\frac{1}{\tau} g_{i j} \nabla_{i} \nabla_{j} f-\frac{\left|g_{i j}\right|^{2}}{4 \tau^{2}}\right) \\
= & -\tau\left(2|\operatorname{Ric}|^{2}+4 R_{i j} \nabla_{i} \nabla_{j} f+2|\operatorname{Hess}(f)|^{2}\right) \\
& +2(R+\triangle f)-\frac{n}{2 \tau} .
\end{aligned}
$$


A direct computation yields

$$
\begin{aligned}
u^{-1} \square^{*} v:= & u^{-1}\left(-\partial_{t}-\triangle+R\right) v \\
= & u^{-1}\left[-\left(\partial_{t}+\triangle\right)\left[\tau\left(2 \triangle f-|\nabla f|^{2}+R\right)+f-n\right]\right] u \\
& -2 u^{-1}\left\langle\nabla\left[\tau\left(2 \triangle f-|\nabla f|^{2}+R\right)+f-n\right], \nabla u\right\rangle \\
& +u^{-1}\left[\tau\left(2 \triangle f-|\nabla f|^{2}+R\right)+f-n\right] \square^{*} u \\
= & -\left(\partial_{t}+\triangle\right)\left[\tau\left(2 \triangle f-|\nabla f|^{2}+R\right)+f-n\right] \\
& +2\left\langle\nabla\left[\tau\left(2 \triangle f-|\nabla f|^{2}+R\right)+f-n\right], \nabla f\right\rangle,
\end{aligned}
$$

where in the last step we used $u^{-1} \nabla u=-\nabla f$ and $\square^{*} u=0$. We continue

$$
\begin{aligned}
u^{-1} \square^{*} v= & \left(2 \triangle f-|\nabla f|^{2}+R\right)-\tau\left(\partial_{t}+\triangle\right)\left(2 \triangle f-|\nabla f|^{2}+R\right) \\
& -\left(\partial_{t}+\triangle\right) f+2 \tau\left\langle\nabla\left(2 \triangle f-|\nabla f|^{2}+R\right), \nabla f\right\rangle+2|\nabla f|^{2} \\
= & 2 \triangle f+|\nabla f|^{2}+R-\tau\left[\left(\partial_{t}+\triangle\right)\left(2 \triangle f-|\nabla f|^{2}+R\right)\right] \\
& -\left(\partial_{t}+\triangle\right) f+2 \tau\left\langle\nabla\left(2 \triangle f-|\nabla f|^{2}+R\right), \nabla f\right\rangle .
\end{aligned}
$$

For the part of (3.23) which is proportional to $\tau$, one obtains

$$
\begin{aligned}
(\star):= & \left(\partial_{t}+\triangle\right)\left(2 \triangle f-|\nabla f|^{2}+R\right)-2\left\langle\nabla\left(2 \triangle f-|\nabla f|^{2}+R\right), \nabla f\right\rangle \\
= & 2 \dot{\triangle} f+2 \triangle\left(\partial_{t}+\triangle\right) f-\left(\partial_{t}+\triangle\right)|\nabla f|^{2}+\left(\partial_{t}+\triangle\right) R \\
& -2\left\langle\nabla\left(2 \triangle f-|\nabla f|^{2}+R\right), \nabla f\right\rangle .
\end{aligned}
$$

With the evolution equations $\partial_{t} R=\triangle R+2 \mid$ Ric $\left.\right|^{2}$ and $\dot{\triangle} f=2 R_{i j} \nabla_{i} \nabla_{j} f$ from chapter one, and with (3.21), we continue

$$
\begin{aligned}
(\star)= & 4 R_{i j} \nabla_{i} \nabla_{j} f+2 \triangle\left(|\nabla f|^{2}-R\right)-2 \operatorname{Ric}(\nabla f, \nabla f) \\
& -2\left\langle\nabla\left(\partial_{t} f\right), \nabla f\right\rangle-\triangle|\nabla f|^{2}+2 \triangle R+2|\operatorname{Ric}|^{2} \\
& -2\left\langle\nabla\left(2 \triangle f-|\nabla f|^{2}+R\right), \nabla f\right\rangle \\
= & 2|\operatorname{Ric}|^{2}+4 R_{i j} \nabla_{i} \nabla_{j} f+\triangle|\nabla f|^{2}-2 \operatorname{Ric}(\nabla f, \nabla f) \\
& -2\left\langle\nabla\left(\partial_{t} f+2 \triangle f-|\nabla f|^{2}+R\right), \nabla f\right\rangle .
\end{aligned}
$$

Using again the evolution equation (3.21) for $f$ from above, we find $\left(\partial_{t} f+2 \triangle f-|\nabla f|^{2}+R\right)=$ $\triangle f$ and hence we get

$$
\begin{aligned}
(\star)= & 2|\operatorname{Ric}|^{2}+4 R_{i j} \nabla_{i} \nabla_{j} f+\Delta|\nabla f|^{2} \\
& -2 \operatorname{Ric}(\nabla f, \nabla f)-2\langle\nabla \triangle f, \nabla f\rangle \\
= & 2|\operatorname{Ric}|^{2}+4 R_{i j} \nabla_{i} \nabla_{j} f+2|\operatorname{Hess}(f)|^{2},
\end{aligned}
$$

where in the last step we used once more our formula (2.3) in the form

$$
\triangle|\nabla f|^{2}=2\langle\nabla \triangle f, \nabla f\rangle+2 \operatorname{Ric}(\nabla f, \nabla f)+2|\operatorname{Hess}(f)|^{2} .
$$


For the remaining terms of (3.23), which are not proportional to $\tau$, we find with (3.21)

$$
\begin{aligned}
2 \triangle f+|\nabla f|^{2}+R-\left(\partial_{t}+\triangle\right) f & =2 \triangle f+|\nabla f|^{2}+R-\left(|\nabla f|^{2}-R+\frac{n}{2 \tau}\right) \\
& =2(R+\triangle f)-\frac{n}{2 \tau} .
\end{aligned}
$$

Plugging this and (3.24) into (3.23), we obtain

$$
u^{-1} \square^{*} v=-\tau\left[2|\operatorname{Ric}|^{2}+4 R_{i j} \nabla_{i} \nabla_{j} f+2|\operatorname{Hess}(f)|^{2}\right]+2(R+\triangle f)-\frac{n}{2 \tau},
$$

which is exactly what we wanted to prove.

Definition 3.12 (entropy formula for shrinking solitons)

Let $g_{i j}$ satisfy the Ricci flow equation. We define the shrinker entropy by

$$
\mathcal{W}\left(g_{i j}, u, \tau\right)=\int_{M}\left[\tau\left(|\nabla f|^{2}+R\right)+f-n\right] u d V
$$

restricted to u satisfying $\int_{M} u d V=1$ and $\tau=(T-t)>0$. We also define its local version

$$
v=W u=\left[\tau\left(2 \triangle f-|\nabla f|^{2}+R\right)+f-n\right] u .
$$

With lemma 3.11 above, we get the monotonicity

$$
\begin{aligned}
\frac{d}{d t} \mathcal{W}(g, u, \tau) & =\partial_{t} \int_{M} W u d V=-\int_{M} \square^{*}(W u) d V \\
& =\int_{M} 2 \tau\left|\operatorname{Ric}+\operatorname{Hess}(f)-\frac{g}{2 \tau}\right|^{2} u d V \geq 0
\end{aligned}
$$

and $\frac{d}{d t} \mathcal{W}(g, u, \tau)=0$ exactly for gradient shrinking solitons with potential $f$, which satisfy $R_{i j}+\nabla_{i} \nabla_{j} f-\frac{1}{2 \tau} g_{i j}=0$. So, similar to the steady soliton case, the functional is nondecreasing and constant exactly on shrinkers.

The local version satisfies again a pointwise estimate for the (adjoint) kernel. The following result may be proved in much the same way as proposition 3.6.

Proposition 3.13 (Perelman, corollary 9.3 of [28])

If $u$ tends to a $\delta$-function as $t \rightarrow T$, then $W \leq 0$ for all $t<T$.

Proof. As in the heat equation case, choose an arbitrary $t_{0}<T$ and $h\left(\cdot, t_{0}\right)$ smooth and positive. Let $h(\cdot, t)$ be a solution to the heat equation $\square h=0$ for $t \geq t_{0}$. Then we have

$$
\partial_{t} \int_{M} h u d V=\int_{M}\left((\square h) u-h\left(\square^{*} u\right)\right) d V=0,
$$

as well as

$$
\partial_{t} \int_{M} h v d V=\int_{M}\left((\square h) v-h\left(\square^{*} v\right)\right) d V=-\int_{M} h \square^{*} v d V \geq 0, \quad \forall t<T .
$$

Hence to prove the proposition, it remains to show that $v=W u$ satisfies

$$
\lim _{t \rightarrow T} \int_{M} h v \leq 0 .
$$


This can be proved with a similar argument to the proof of proposition 3.6 above, the computations being slightly more complicated. To shorten the text, we skip the proof of this inequality and refer the reader to Lei Ni's paper [27]. However, some of the theory used in Lei Ni's proof will only be explained in chapter four of this book.

We know that the entropy $\mathcal{W}(g, u, \tau)$ is non-decreasing in time. But what about the local entropy $W$ ? We can show that at least the maximum value of $W$ over $M$ also increases, see [28], section 9.

Proposition 3.14 (Perelman, corollary 9.2 of [28])

On the closed manifold $M$ the maximum value of $W=\frac{v}{u}$ is non-decreasing in $t$.

Proof. On a maximum point of $W$, we have $\triangle W \leq 0$. We first compute $\left(\partial_{t}+\triangle\right) W$ to get an estimate on $\partial_{t} W$. We have

$$
\begin{aligned}
\left(\partial_{t}+\triangle\right) \frac{v}{u} & =\partial_{t}\left(\frac{v}{u}\right)+\triangle\left(v \cdot u^{-1}\right) \\
& =\frac{u \partial_{t} v-v \partial_{t} u}{u^{2}}+u^{-1} \triangle v+v \triangle u^{-1}+2\left\langle\nabla v, \nabla u^{-1}\right\rangle .
\end{aligned}
$$

With $\nabla u^{-1}=-\frac{\nabla u}{u^{2}}$ we find that the last expression in (3.26) equals

$$
2\left\langle\nabla v, \nabla u^{-1}\right\rangle=-\frac{2}{u^{3}}\langle u \nabla v, \nabla u\rangle .
$$

Furthermore, with

$$
\triangle u^{-1}=\operatorname{div}\left(-\frac{\nabla u}{u^{2}}\right)=\left(-\frac{\triangle u}{u^{2}}+\frac{2}{u^{3}}\langle\nabla u, \nabla u\rangle\right),
$$

we get

$$
v \triangle u^{-1}=-\frac{v \triangle u}{u^{2}}+\frac{2}{u^{3}}\langle\nabla u, v \nabla u\rangle .
$$

Inserting these two expressions into (3.26) yields

$$
\begin{aligned}
\left(\partial_{t}+\triangle\right) \frac{v}{u}= & \frac{u \partial_{t} v-v \partial_{t} u}{u^{2}}+\frac{u \triangle v-v \triangle u}{u^{2}} \\
& +\frac{2}{u^{3}}\langle\nabla u, v \nabla u\rangle-\frac{2}{u^{3}}\langle\nabla u, u \nabla v\rangle \\
= & \frac{v \square^{*} u-u \square^{*} v}{u^{2}}-\frac{2}{u^{3}}\langle\nabla u,(u \nabla v-v \nabla u)\rangle .
\end{aligned}
$$

Because we compute this at a maximum point, where $0=\nabla \frac{v}{u}=\frac{1}{u^{2}}(u \nabla v-v \nabla u)$, the scalar product term in (3.27) is zero. With $\square^{*} u=0, \square^{*} v \leq 0$ and $u>0$, we conclude that

$$
\left(\partial_{t}+\triangle\right) \frac{v}{u}=\frac{v \square^{*} u}{u^{2}}-\frac{u \square^{*} v}{u^{2}} \geq 0 .
$$

This yields $\partial_{t} W=\partial_{t} \frac{v}{u} \geq-\triangle \frac{v}{u} \geq 0$ at a maximum point, which proves the claim.

Finally, we prove a differential Harnack inequality for $f$ which we can integrate along a path, similarly to the static case, proposition 3.9. The following result is a slightly modified version of corollary 9.4 from [28]. 
Proposition 3.15 (Perelman's differential Harnack inequality)

Assume that $u$ is a positive solution of the adjoint heat equation with tends to a $\delta$-function as $t \rightarrow T$. We write $u$ in the form $u(x, \tau)=(4 \pi \tau)^{-n / 2} e^{-f(x, \tau)}$. Then for any smooth curve $\gamma(\tau)$ in $M$, we obtain

$$
\frac{d}{d \tau} f(\gamma(\tau), \tau) \leq \frac{1}{2}\left(R(\gamma(\tau), \tau)+|\dot{\gamma}(\tau)|^{2}\right)-\frac{f(\gamma(\tau), \tau)}{2 \tau} .
$$

Proof. With proposition 3.13 we get $W \leq 0$ which is equivalent to

$$
-\triangle f+\frac{1}{2}|\nabla f|^{2}-\frac{1}{2} R+\frac{n-f}{2 \tau} \geq 0 .
$$

If we insert (3.21) in the form

$$
\triangle f=\partial_{\tau} f+\frac{n}{2 \tau}+|\nabla f|^{2}-R
$$

into the above equation, we get

$$
-\partial_{\tau} f-\frac{1}{2}|\nabla f|^{2}+\frac{1}{2} R-\frac{f}{2 \tau} \geq 0 .
$$

We can then compute

$$
\begin{aligned}
\frac{d}{d \tau} f(\gamma(\tau), \tau) & =\partial_{\tau} f(\gamma(\tau), \tau)+\langle\nabla f(\gamma(\tau), \tau), \dot{\gamma}(\tau)\rangle \\
& \leq \partial_{\tau} f+\frac{1}{2}|\nabla f|^{2}+\frac{1}{2}|\dot{\gamma}|^{2} \leq \frac{1}{2} R+\frac{1}{2}|\dot{\gamma}|^{2}-\frac{f}{2 \tau},
\end{aligned}
$$

where we added the positive expression (3.29) in the last step.

Again, we can not directly integrate this, so we multiply the function $f$ in the inequality of the proposition with $2 \sqrt{\tau}$. We can then write all terms containing $f$ as one single derivative, namely

$$
\frac{d}{d \tau}(2 \sqrt{\tau} f(\gamma(t), t))=\frac{f(\gamma(t), t)}{\sqrt{\tau}}+2 \sqrt{\tau} \frac{d}{d \tau} f(\gamma(t), t) \leq \sqrt{\tau}\left(R(\gamma(\tau), \tau)+|\dot{\gamma}(\tau)|^{2}\right) .
$$

We integrate along the path $\gamma(\tau)$ from $\gamma(0)=x$ to $\gamma(\bar{\tau})=y$. This yields

$$
2 \sqrt{\bar{\tau}} f(y, \bar{\tau})=\left.2 \sqrt{\tau} f(\gamma(\tau), \tau)\right|_{0} ^{\bar{\tau}}=\int_{0}^{\bar{\tau}} \frac{d}{d \tau}(2 \sqrt{\tau} f) d \tau \leq \int_{0}^{\bar{\tau}} \sqrt{\tau}\left(R+|\dot{\gamma}|^{2}\right) d \tau .
$$

We get the analog to corollary 3.10 .

Corollary 3.16 (integrated version)

Let $g_{i j}$ be a solution of the Ricci flow $\partial_{t} g=-2$ Ric. Let $u$ be a positive solution of the adjoint heat equation which tends to a $\delta$-function as $t \rightarrow T$ at a point $p \in M$. Then we have the lower bound

$$
u(q, \bar{\tau}) \geq(4 \pi \bar{\tau})^{-n / 2} e^{-\ell(q, \bar{\tau})},
$$

where

$$
\ell(q, \bar{\tau}):=\inf _{\gamma \in \Gamma}\left\{\frac{1}{2 \sqrt{\bar{\tau}}} \int_{0}^{\bar{\tau}} \sqrt{\tau}\left(R+|\dot{\gamma}|^{2}\right) d \tau\right\}
$$

with

$$
\Gamma=\{\gamma:[0, \bar{\tau}] \rightarrow M \mid \gamma(0)=p, \gamma(\bar{\tau})=q\}
$$


Definition 3.17 (backwards reduced volume)

We call the above $\ell(q, \bar{\tau})$ the backwards reduced distance between $p$ and $q$. The integral over all $q$,

$$
\tilde{V}(\tau):=\int_{M}(4 \pi \tau)^{-n / 2} e^{-\ell(q, \bar{\tau})} d V(q),
$$

is called the backwards reduced volume of $M$ with basepoint $p$.

One of our main goals in chapter four is to prove the monotonicity of this backwards reduced volume. We will see that $\tilde{V}(\tau)$ is monotone in general and constant exactly on shrinking solitons.

\subsection{Entropy For Ricci expanders}

We leave away all the details here and only write down the results, which can be proved similarly to the shrinking case, we only have to tweak some signs. Let again $\sigma$ denote $(t-T)$ as in chapter 1 . For positive solutions $u=(4 \pi \sigma)^{-n / 2} e^{-f}$ of the adjoint heat equation, which satisfy $\int_{M} u d V=1$, we define for $t>T$ the expander entropy functional by

$$
\mathcal{W}_{+}\left(g_{i j}, u, \sigma\right):=\int_{M}\left[\sigma\left(|\nabla f|^{2}+R\right)-f+n\right] u d V
$$

and its local version by

$$
v_{+}:=W_{+} u=\left[\sigma\left(2 \triangle f-|\nabla f|^{2}+R\right)-f+n\right] u .
$$

Similar to lemma 3.11 above, we find that $v_{+}$satisfies the equation

$$
\square^{*} v_{+}=2 \sigma\left|\operatorname{Ric}+\operatorname{Hess}(f)+\frac{g}{2 \sigma}\right|^{2} u, \quad \text { for } t>T .
$$

This leads to the evolution equation for the entropy $\mathcal{W}_{+}$. One obtains

$$
\frac{d}{d t} \mathcal{W}_{+}(g, u, \sigma)=\int_{M} 2 \sigma\left|\operatorname{Ric}+\operatorname{Hess}(f)+\frac{g}{2 \sigma}\right|^{2} u d V \geq 0,
$$

and $\frac{d}{d t} \mathcal{W}_{+}=0$ exactly for gradient expanding solitons with soliton potential $f$, which satisfy $R_{i j}+\nabla_{i} \nabla_{j} f+\frac{1}{2 \sigma} g_{i j}=0$, see proposition 1.16.

One can find similar results to the shrinking case and to Ni's entropy for the heat equation on a static manifold. For example we can define $\tilde{N}(u, \sigma)=N(u)+\frac{n}{2} \log (4 \pi \sigma)+\frac{n}{2}$, where $N(u)$ denotes the Nash entropy as before. Feldman, Ilmanen and $\mathrm{Ni}$ [12] proved that with $\tilde{F}(u, \sigma)=\frac{d}{d t} \tilde{N}(u, \sigma)$, we can write the entropy formula in the expanding case as

$$
\mathcal{W}_{+}(g, u, \sigma)=\tilde{N}(u, \sigma)+t \tilde{F}(u, \sigma)=\frac{d}{d t}(t \tilde{N}(u, \sigma)),
$$

exactly as in the static manifold case, only with a different sign. This sign change comes from the fact that $u$ satisfies the adjoint heat equation, wich can be regarded as a backwards heat equation with potential $R$. All details can be found in [12].

Remark. With some sign changes, one also gets a similar volume formula to the reduced volume $\tilde{V}(\tau)$ from definition 9, the so-called forward reduced volume. The forward reduced 
volume is monotone in general and constant on expanders.

For a solution $g$ of the Ricci flow, there is the conjecture, that $g(t) / t$ converges in some weak sense to a Ricci negative Einstein manifold as $t \rightarrow \infty$. If the limit is known a-priori to be smooth and compact, this conjecture follows from considering any monotone quantity that is constant on expanders. For instance, one can use this forward reduced volume to prove the conjecture in this special case. However the existence of such quantities was known before. Other monotone functionals which are constant on expanding solitons and can hence be considered to prove the above claim are for example $t^{-n / 2} \operatorname{vol}(g)$ (Hamilton) or $\bar{\lambda}$ (Perelman, section 2.3. of [28]).

\section{Reduced distance and reduced volume}

Motivated by corollary 3.16, we will study the backwards reduced distance $\ell(q, \bar{\tau})$ and its associated backwards reduced volume (see definition 3.17) in this chapter. The main goal is to show that the backwards reduced distance $\ell(q, \tau)$ solves $\square^{*}\left[(4 \pi \tau)^{-n / 2} e^{-\ell(q, \tau)}\right] \leq 0$ and that the backwards reduced volume $\tilde{V}(\tau)$ is non-increasing in $\tau$ under the Ricci flow $\partial_{t} g=-2$ Ric, where $\tau=-t$. To prove this, we will closely follow section 7 of Perelman's paper [28]. Note that the backwards reduced distance is only Lipschitz in general, as will be seen in lemma 4.8 .

The scalar curvature $R$ and the norm $|\dot{\gamma}|^{2}$ in the definition of the backwards reduced distance $\ell(q, \bar{\tau})$ are computed at $\gamma(\tau)$ using the current metric $g_{i j}(\tau)$, i.e. if we move on the curve, the metric on the manifold changes continuously. This makes some of the computations rather long and unclear, and the notations - we try to adopt Perelman's notations here are not always easy to understand. That is why we start again with the static case, where the metric $g_{i j}(t) \equiv g_{i j}(0)$ on $M$ is kept fixed and the corresponding distance functional $\ell(q, t)$ from corollary 3.10 takes a simpler form. However, most of the analytic ideas can already be seen in this simpler case. We prove that on a manifold $M$ with Ric $\geq 0$ the function $u=(4 \pi t)^{-n / 2} e^{-\ell(q, t)}$ is a subsolution of the heat equation. We will also show that the associated reduced volume to the distance function $\ell(q, t)$ is non-increasing in time. If Ric $=0$, this result and Perelman's result coincide.

The evolution of length (and energy) functionals under Ricci flow has already been studied by Hamilton. Namely, he showed that the standard length functional $L(\gamma)=\int_{s_{1}}^{s_{2}}|\gamma(s)| d s$ of an orientation-preserving closed geodesic curve $\gamma$ on a surface satisfies some kind of heat equation under the Ricci flow $\partial_{t} g_{i j}(t)=-2 R_{i j}$. With this, he could prove that a weakly stable geodesic loop on a surface which preserves orientation has its length increase. He also proved a similar result for orientation preserving loops on three-manifolds, which one can generalize to arbitrary dimensions. However, there is an important difference to Perelman's results presented here, because Hamilton only considered curves, where the curve parameter $s$ is independent of the time parameter $t$, with which the metric evolves.

We will not study Hamilton's results here. A good overview of his evolution equations for closed geodesic loops can be found in [19], section 12 .

The way we found the backwards reduced distance in corollary 3.16 suggests that there must be a connection between the $\mathcal{L}$-length and shrinking solitons. One does not only find this, but also a beautiful connection to Hamilton's Harnack estimates for the Ricci flow. 
However, one does not need weakly positive curvature operator, because the Harnack inequalities do not need to be satisfied for the computations here. The Harnack expressions from theorem 2.15 will only appear during the computations and cancel out in the final results.

\subsection{The static case}

The following results about the first and second variation of the energy functional are classical in Riemannian geometry, cf. [21], but we will introduce a different notation, which is adopted from Perelman's paper [28]. Let $g_{i j}$ be a fixed Riemannian metric on the closed manifold $M$. The standard energy functional for a curve $\gamma:\left[t_{0}, T\right] \rightarrow M$ is defined by

$$
E(\gamma):=\int_{t_{0}}^{T} \frac{1}{2}|\dot{\gamma}(t)|^{2} d t
$$

Let $\gamma_{s}(\cdot)=\gamma(s, \cdot)$ be a variation of $\gamma(t)=\gamma_{0}(t)$, i.e. $\gamma:(-\varepsilon, \varepsilon) \times\left[t_{0}, T\right] \rightarrow M$ smooth with $\gamma(0, t)=\gamma_{0}(t), \forall t \in\left[t_{0}, T\right]$. We introduce the notation $X(t):=\left.\partial_{t} \gamma_{s}(t)\right|_{s=0}$ and $Y(t):=\left.\partial_{s} \gamma_{s}(t)\right|_{s=0}$. We write $\nabla_{X}:=\tilde{\nabla}_{t}$ and $\nabla_{Y}:=\tilde{\nabla}_{s}$, where $\tilde{\nabla}$ denotes the pull-back connection of the Levi-Cività connection on $M$. In this notation, we get the first variation of the energy functional,

$$
\delta_{Y} E(\gamma):=\left.\partial_{s} E\left(\gamma_{s}\right)\right|_{s=0}=\left.\langle Y, X\rangle\right|_{t_{0}} ^{T}-\int_{t_{0}}^{T}\left\langle Y, \nabla_{X} X\right\rangle d t
$$

A curve $\gamma$ is called geodesic, if the first variation of $E(\gamma)$ vanishes for all variations $\gamma_{s}$ with fixed endpoints, i.e. variations with $Y\left(t_{0}\right)=Y(T)=0$. Hence $\gamma$ is a geodesic iff $\nabla_{X} X=0$. For geodesic curves, we get the second variation

$$
\delta_{Y}^{2} E(\gamma):=\left.\left\langle\nabla_{Y} Y, X\right\rangle\right|_{t_{0}} ^{T}+\int_{t_{0}}^{T}\left(\left|\nabla_{X} Y\right|^{2}-\langle R(Y, X) X, Y\rangle\right) d t .
$$

Now let $u$ be the positive heat kernel on $M$ and let $p \in M$ be the point where the $\delta$-function forms for $t \rightarrow 0$. By integrating a differential Harnack inequality, we showed in corollary 3.10 that the heat kernel $u$ on $M$ satisfies the inequality

$$
u(q, T) \geq(4 \pi T)^{-n / 2} e^{-\ell(q, T)},
$$

where

$$
\ell(q, T):=\inf _{\gamma \in \Gamma}\left\{\frac{1}{2 \sqrt{T}} \int_{0}^{T} \sqrt{t}|\dot{\gamma}|^{2} d t\right\}
$$

with

$$
\Gamma:=\{\gamma:[0, T] \rightarrow M \mid \gamma(0)=p, \gamma(T)=q\} .
$$

To study this reduced distance $\ell(q, T)$ we introduce the length functional

$$
\mathcal{L}(\gamma):=\int_{t_{0}}^{T} \sqrt{t}|\dot{\gamma}(t)|^{2} d t
$$

for a curve $\gamma(t):\left[t_{0}, T\right] \rightarrow M$. Let $\gamma_{s}$ be a variation of $\gamma$, as above. We use again the notation $X(t)=\left.\partial_{t} \gamma_{s}(t)\right|_{s=0}$ and $Y(t)=\left.\partial_{s} \gamma_{s}(t)\right|_{s=0}$. We compute the first variation of the 
L-length,

$$
\begin{aligned}
\delta_{Y} \mathcal{L}(\gamma) & =\int_{t_{0}}^{T} 2 \sqrt{t}\left\langle\nabla_{Y} X, X\right\rangle d t=\int_{t_{0}}^{T} 2 \sqrt{t}\left\langle\nabla_{X} Y, X\right\rangle d t \\
& =2 \int_{t_{0}}^{T} \sqrt{t}\left(\partial_{t}\langle Y, X\rangle-\left\langle Y, \nabla_{X} X\right\rangle\right) d t \\
& =2 \int_{t_{0}}^{T}\left(\partial_{t}(\sqrt{t}\langle Y, X\rangle)-\frac{1}{2 \sqrt{t}}\langle Y, X\rangle-\sqrt{t}\left\langle Y, \nabla_{X} X\right\rangle\right) d t \\
& =\left.2 \sqrt{t}\langle Y, X\rangle\right|_{t_{0}} ^{T}-2 \int_{t_{0}}^{T} \sqrt{t}\left\langle Y, \nabla_{X} X+\frac{1}{2 t} X\right\rangle d t
\end{aligned}
$$

which implies that $\mathcal{L}$-geodesics must satisfy

$$
G(X):=\nabla_{X} X+\frac{1}{2 t} X=0 .
$$

Note that the substitution $\lambda=\sqrt{t}$ yields

$$
\mathcal{L}(\gamma)=\int_{t_{0}}^{T} \sqrt{t}|\dot{\gamma}(t)|^{2} d t=\int_{\sqrt{t_{0}}}^{\sqrt{T}} 2 \lambda^{2}\left|\dot{\gamma}\left(\lambda^{2}\right)\right|^{2} d \lambda=\int_{\sqrt{t_{0}}}^{\sqrt{T}} \frac{1}{2}\left|\gamma^{\prime}(\lambda)\right|^{2} d \lambda,
$$

where $\dot{\gamma}(t)=\partial_{t} \gamma(t), \gamma^{\prime}(\lambda)=\partial_{\lambda} \gamma(\lambda)$. In other words, the $\mathcal{L}$-length functional is a reparametrization of the standard energy functional above. With the notation $\tilde{X}(\lambda)=\gamma^{\prime}(\lambda)$, we find that (4.2) is equivalent to $\nabla_{\tilde{X}} \tilde{X}=0$, i.e. $\gamma(t)$ is an $\mathcal{L}$-geodesic if and only if $\gamma(\lambda)=\gamma(\sqrt{t})$ is an $E$-geodesic.

If $\gamma(t)$ is an $\mathcal{L}$-geodesic, equation (4.2) implies

$$
\partial_{t}|X|^{2}=2\left\langle\nabla_{X} X, X\right\rangle=-\frac{1}{t}|X|^{2},
$$

i.e. $t|X(t)|^{2}$ is constant and hence $\sqrt{t} X(t)$ has a limit as $t \rightarrow 0$ for geodesic curves. So in this case we can extend the definition of $\mathcal{L}(\gamma)$ above to $t_{0}=0$.

For an $\mathcal{L}$-geodesic $\gamma:[0, T] \rightarrow M$, the second variation can be computed as

$$
\delta_{Y}^{2} \mathcal{L}(\gamma)=\left.2 \sqrt{T}\left\langle\nabla_{Y} Y, X\right\rangle\right|_{0} ^{T}+2 \int_{0}^{T} \sqrt{t}\left(\left|\nabla_{X} Y\right|^{2}-\langle R(Y, X) X, Y\rangle\right) d t .
$$

We now fix $p \in M$ and denote by $L(q, T)$ the $\mathcal{L}$-length of an $\mathcal{L}$-shortest geodesic from $p=\gamma(0)$ to $q=\gamma(T)$. We can hence write the reduced distance $\ell(q, T)$ from corollary 3.10 as $\ell(q, T)=\frac{1}{2 \sqrt{T}} L(q, T)$. Because of (4.3), $\gamma$ is an $E$-geodesic, and we can compute

$$
L(q, T)=\frac{1}{\sqrt{T}} \cdot \frac{d^{2}(p, q)}{2},
$$

and thus $\ell(q, T)=\frac{d^{2}(p, q)}{4 T}$. The regularity for $\mathcal{L}$ is established only in the Ricci flow case below, but analogous arguments work also for the static case. 
Lemma 4.1 (equations for the reduced distance)

The reduced distance $\ell(q, T)$ satisfies the following equations.

$$
\begin{aligned}
|\nabla \ell(q, T)|^{2} & =\frac{1}{T} \ell(q, T), \\
\partial_{T} \ell(q, T) & =-\frac{1}{T} \ell(q, T), \\
\triangle \ell(q, T) & \leq \frac{n}{2 T}-\frac{1}{2 T^{3 / 2}} \int_{0}^{T} 2 t^{3 / 2} \operatorname{Ric}(X, X) d t .
\end{aligned}
$$

Proof. We multiply (4.4) by $t^{3 / 2}$ and integrate along $\gamma$. This yields

$$
\begin{aligned}
-L(q, T) & =-\int_{0}^{T} \sqrt{t}|X|^{2} d t=\int_{0}^{T} t^{3 / 2} \partial_{t}|X|^{2} d t \\
& =\int_{0}^{T}\left(\partial_{t}\left(t^{3 / 2}|X|^{2}\right)-\frac{3}{2} \sqrt{t}|X|^{2}\right) d t \\
& =\left.t^{3 / 2}|X|^{2}\right|_{0} ^{T}-\frac{3}{2} \int_{0}^{T} \sqrt{t}|X|^{2} d t=T^{3 / 2}|X(T)|^{2}-\frac{3}{2} L(q, T),
\end{aligned}
$$

and hence

$$
\frac{1}{2} L(q, T)=T^{3 / 2}|X(T)|^{2}
$$

Since an $\mathcal{L}$-geodesic $\gamma$ satisfies (4.2), the first variation of $\mathcal{L}(\gamma)$ becomes simply $\delta_{Y} L=$ $\left.2 \sqrt{t}\langle Y, X\rangle\right|_{0} ^{T}$. Note that this depends only on the value of $Y$ at $t=T$. Hence $\nabla_{Y} L=\delta_{Y} L$, where $Y$ in the first term denotes $Y(T)$ whereas in the second term it denotes the vector field $Y(t)$ along the curve $\gamma(t)$. With this, we obtain $\nabla L(q, T)=2 \sqrt{T} X(T)$. Equation (4.8) yields

$$
|\nabla L(q, T)|^{2}=4 T|X(T)|^{2}=\frac{2}{\sqrt{T}} L(q, T) .
$$

Hence

$$
|\nabla \ell(q, T)|^{2}=\frac{1}{4 T}|\nabla L(q, T)|^{2}=\frac{1}{2 T^{3 / 2}} L(q, T)=\frac{1}{T} \ell(q, T),
$$

which proves (4.5). Note that $\frac{d}{d T} L(q, T)$ has a time direction component $\partial_{T} L(q, T)$ and a space direction component $\nabla_{X} L(q, T)$. With equation (4.8) and $L(q, T)=\int_{0}^{T} \sqrt{t}|X(t)|^{2} d t$, we find

$$
\begin{aligned}
\partial_{T} L(q, T) & =\frac{d}{d T} L(q, T)-\nabla_{X} L(q, T)=\sqrt{T}|X(T)|^{2}-\langle X(T), \nabla L(q, T)\rangle \\
& =-\sqrt{T}|X|^{2}=-\frac{1}{2 T} L(q, T),
\end{aligned}
$$

and hence

$$
\partial_{T} \ell(q, T)=\partial_{T}\left(\frac{1}{2 \sqrt{T}} L(q, T)\right)=-\frac{L(q, T)}{4 T^{3 / 2}}-\frac{L(q, T)}{4 T^{3 / 2}}=-\frac{1}{T} \ell(q, T),
$$

which is exactly (4.6). Now let $Y(T)$ be any normalized tangent vector in $T_{q} M$, i.e. $|Y(T)|=1$, and define $Y(t)$ as the solution of

$$
\nabla_{X} Y=\frac{1}{2 t} Y
$$


This immediately implies $|Y(t)|^{2}=t / T$, in particular $Y(0)=0$. Then there is a smooth vector field $\tilde{Y}(t)$ along $\gamma$ with $|\tilde{Y}(t)| \equiv 1$ and $Y(t)=\sqrt{t / T} \tilde{Y}(t)$. With a variation $\gamma_{s}(t)$ in the direction $Y(t)$, we find

$$
\begin{aligned}
\operatorname{Hess}_{L}(Y(T), Y(T)) & \leq \operatorname{Hess}_{\mathcal{L}}(Y, Y)=2 \int_{0}^{T} \sqrt{t}\left(\left|\nabla_{X} Y\right|^{2}-\langle R(Y, X) X, Y\rangle\right) d t \\
& =\int_{0}^{T}\left(\frac{1}{2 T \sqrt{t}}-2 \sqrt{t}\langle R(Y, X) X, Y\rangle\right) d t \\
& =\frac{1}{\sqrt{T}}-\int_{0}^{T} \frac{2 t^{3 / 2}}{T}\langle R(\tilde{Y}, X) X, \tilde{Y}\rangle d t
\end{aligned}
$$

Summing over an orthonormal basis $\left\{Y_{1}, \ldots, Y_{n}\right\}$ of $T_{q} M$ and the associated vector fields $\left\{\tilde{Y}_{1}(t), \ldots, \tilde{Y}_{n}(t)\right\}$ along $\gamma$ yields

$$
\begin{aligned}
\triangle L(q, T) & =\sum_{i=1}^{n} \operatorname{Hess}_{L}\left(Y_{i}, Y_{i}\right) \\
& \leq \frac{n}{\sqrt{T}}-\frac{1}{T} \int_{0}^{T} 2 t^{3 / 2}\left(\sum_{i=1}^{n}\left\langle R\left(\tilde{Y}_{i}, X\right) X, \tilde{Y}_{i}\right\rangle\right) d t \\
& =\frac{n}{\sqrt{T}}-\frac{1}{T} \int_{0}^{T} 2 t^{3 / 2} \operatorname{Ric}(X, X) d t
\end{aligned}
$$

where for the last step we used (1.1) and the Gauss lemma which says that $\tilde{Y}_{i}(t)$ stay orthonormal for all $t$. So for $\ell(q, T)$, we find

$$
\triangle \ell(q, T)=\frac{1}{2 \sqrt{T}} \triangle L \leq \frac{n}{2 T}-\frac{1}{2 T^{3 / 2}} \int_{0}^{T} 2 t^{3 / 2} \operatorname{Ric}(X, X) d t,
$$

which is equation (4.7) of the lemma.

Note that for a manifold $M$ with Ric $\geq 0$ equation (4.7) becomes $\triangle \ell(q, t) \leq \frac{n}{2 t}$, which is exactly the Li-Yau Harnack inequality for a (sub)solution $v(q, t):=(4 \pi t)^{-n / 2} e^{-\ell(q, t)}$ of the heat equation. We now show that the function $v(q, t)$ defined this way satisfies indeed $\square v \leq 0$.

\section{Proposition 4.2}

Let $M$ be a closed manifold with Ric $\geq 0$. Let $v(q, t):=(4 \pi t)^{-n / 2} e^{-\ell(q, t)}$ where $\ell(q, t)$ is the reduced distance defined above. Then $v(q, t)$ is a subsolution to the heat equation, i.e. $\square v=\partial_{t} v-\triangle v \leq 0$.

Proof. As mentioned above, equation (4.7) becomes $\triangle \ell(q, t) \leq \frac{n}{2 t}$ in the case where Ric $\geq 0$. Together with the two equations (4.5) and (4.6), we get

$$
\partial_{t} \ell(q, t)-\triangle \ell(q, t)+|\nabla \ell(q, t)|^{2}+\frac{n}{2 t} \geq 0,
$$

which is apparently equivalent to $\square v \leq 0$.

We now have the necessary material to prove equation (3.14) from chapter three. 


\section{Corollary 4.3}

If Ric $\geq 0$ and $u=(4 \pi t)^{-n / 2} e^{-f}$ is the heat kernel which tends to a $\delta$-function at $p \in M$ for $t \rightarrow 0$, then $f(q, t) \leq d^{2}(p, q) / 4 t$.

Proof. Note that we are not allowed to use $f \leq \ell$ from corollary 3.10, since we used equation (3.14) to prove this corollary. But for $\ell(q, t)=d^{2}(p, q) / 4 t$ the function $v(q, t)=$ $(4 \pi t)^{-n / 2} e^{-\ell(q, t)}$ tends to the same $\delta$-function at $p \in M$ as $u$ does, and according to proposition 4.2 we have $\square v \leq 0$, so we get $v \leq u$ on $M_{T}$ with the maximum principle and hence $f \leq \ell$.

We define the reduced volume as

$$
\tilde{V}(t)=\int_{M}(4 \pi t)^{-n / 2} e^{-\ell(q, t)} d V(q)=\int_{M} v(q, t) d V(q) .
$$

Proposition 4.2 implies that

$$
\partial_{t} \tilde{V}(t)=\int_{M} \partial_{t} v(q, t) d V(q) \leq \int_{M} \triangle v(q, t) d V(q)=0,
$$

and hence the reduced volume is non-increasing in time. Note that the classical volume of $M$ is of course constant, because $g_{i j}$ is constant, but the reduced distance depends on $t$, because $\mathcal{L}$-geodesics $\gamma:[0, t] \rightarrow M$ have an $\mathcal{L}$-length which depends on $t$.

Remark. If $M$ is the Euclidean space $\mathbb{R}^{n}$ and $p=0$ is the origin, we have $\ell(q, t)=\frac{|q|^{2}}{4 t}$, and so $v(q, t)=(4 \pi t)^{-n / 2} e^{-\ell(q, t)}$ is not only a lower bound to the heat kernel (which is true on every closed manifold $M$ with Ric $\geq 0$ according to the corollaries 3.10 and 4.3), but it is in fact equal to the heat kernel. This is in fact the only case where $\ell(q, t)=f(q, t)$ holds. We can see that with the derivation of $\ell(q, t)$ by integrating the Harnack inequality from proposition 3.9, which must hold as an equality to get $\ell(q, t)=f(q, t)$. However, this Harnack inequality can only hold as equality if (3.19) holds as equality, which implies that $M$ is isometric to $\mathbb{R}^{n}$, see theorem 3.8 .

\subsection{Perelman's $\mathcal{L}$-length and $\mathcal{L}$-geodesics}

We now do the analogous computations for the Ricci flow case. In the remainder of this chapter, we let $\tau:=-t$, so that $g_{i j}(\tau)$ satisfies the backwards Ricci flow in $\tau, \partial_{\tau} g=2$ Ric. We assume again that $M$ is closed or alternatively that all $\left(M, g_{i j}(\tau)\right)$ are complete and have uniformly bounded curvatures.

Definition 4.4 (Perelman's $\mathcal{L}$-functional)

Let $\gamma:\left[\tau_{0}, \bar{\tau}\right] \rightarrow M$ be a smooth curve with $0<\tau_{0}<\bar{\tau}$. We define the $\mathcal{L}$-length of $\gamma(\tau)$ as

$$
\mathcal{L}(\gamma):=\int_{\tau_{0}}^{\bar{\tau}} \sqrt{\tau}\left(R(\gamma(\tau))+|\dot{\gamma}(\tau)|^{2}\right) d \tau
$$

where $\dot{\gamma}(\tau):=\partial_{\tau} \gamma(\tau)$. Note that $R(\gamma(\tau))$ and $|\dot{\gamma}(\tau)|^{2}$ are computed using the metric at time $\tau$.

Remark. While one moves on the curve $\gamma(\tau)$, the metric changes continuously, so $\gamma$ may be seen as a path on the space-time manifold $\tilde{M}$ and $\mathcal{L}$ as a functional on the space of space-time curves. 
The change of variable $\lambda=\sqrt{\tau}$ yields

$$
\mathcal{L}(\gamma)=\int_{\sqrt{\tau_{0}}}^{\sqrt{\bar{\tau}}} 2 \lambda^{2}\left(R(\gamma(\lambda))+|\dot{\gamma}(\lambda)|^{2}\right) d \lambda=\int_{\sqrt{\tau_{0}}}^{\sqrt{\bar{\tau}}}\left(2 \lambda^{2} R(\gamma(\lambda))+\frac{1}{2}\left|\gamma^{\prime}(\lambda)\right|^{2}\right) d \lambda,
$$

where $\gamma^{\prime}(\lambda)=\partial_{\lambda} \gamma(\lambda)$. This time we do not get the standard energy functional as in the static case, but something which differs from the standard energy functional by the curvature term $2 \lambda^{2} R(\gamma(\lambda))$ in the integrand.

We now take a variation $\gamma_{s}$ of $\gamma$ and define $Y(\tau)=\left.\partial_{s} \gamma_{s}(\tau)\right|_{s=0}$ as above. We also define $X(\tau):=\left.\partial_{t} \gamma_{s}(\tau)\right|_{s=0}$ as in the static case. We compute the first variation of $\mathcal{L}(\gamma)$ in the direction of $Y(\tau)$.

$$
\begin{aligned}
\delta_{Y} \mathcal{L}(\gamma) & :=\left.\partial_{s} \mathcal{L}\left(\gamma_{s}\right)\right|_{s=0}=\left.\int_{\tau_{0}}^{\bar{\tau}} \sqrt{\tau} \partial_{s}\left(R\left(\gamma_{s}(\tau)\right)+\langle X, X\rangle\right)\right|_{s=0} d \tau \\
& =\int_{\tau_{0}}^{\bar{\tau}} \sqrt{\tau}\left(\nabla_{Y} R+2\left\langle\nabla_{Y} X, X\right\rangle\right) d \tau \\
& =\int_{\tau_{0}}^{\bar{\tau}} \sqrt{\tau}\left(\langle Y, \nabla R\rangle+2\left\langle\nabla_{X} Y, X\right\rangle\right) d \tau \\
& =\int_{\tau_{0}}^{\bar{\tau}} \sqrt{\tau}\left(\langle Y, \nabla R\rangle+2 \partial_{\tau}\langle Y, X\rangle-2\left\langle Y, \nabla_{X} X\right\rangle-2 \cdot 2 \operatorname{Ric}(Y, X)\right) d \tau \\
& =\int_{\tau_{0}}^{\bar{\tau}} 2 \sqrt{\tau} \partial_{\tau}\langle Y, X\rangle d \tau+\int_{\tau_{0}}^{\bar{\tau}} \sqrt{\tau}\left\langle Y, \nabla R-2 \nabla_{X} X-4 \operatorname{Ric}(X, \cdot)\right\rangle d \tau,
\end{aligned}
$$

where we used $\partial_{\tau}\langle Y, X\rangle=\left\langle\nabla_{X} Y, X\right\rangle+\left\langle Y, \nabla_{X} X\right\rangle+2 \operatorname{Ric}(Y, X)$. For the first integral we find by partial integration

$$
\int_{\tau_{0}}^{\bar{\tau}} 2 \sqrt{\tau} \partial_{\tau}\langle Y, X\rangle d \tau=\left.2 \sqrt{\tau}\langle Y, X\rangle\right|_{\tau_{0}} ^{\bar{\tau}}-\int_{\tau_{0}}^{\bar{\tau}} \frac{1}{\sqrt{\tau}}\langle Y, X\rangle d \tau
$$

Plugging this into the above computation, we get

$$
\delta_{Y} \mathcal{L}(\gamma)=\left.2 \sqrt{\tau}\langle Y, X\rangle\right|_{\tau_{0}} ^{\bar{\tau}}-2 \int_{\tau_{0}}^{\bar{\tau}} \sqrt{\tau}\langle Y, G(X)\rangle d \tau
$$

where

$$
G(X):=\nabla_{X} X-\frac{1}{2} \nabla R+\frac{1}{2 \tau} X+2 \operatorname{Ric}(X, \cdot) .
$$

In other words, the condition for $\gamma(\tau)$ to be an $\mathcal{L}$-geodesic is

$$
G(X)=\nabla_{X} X-\frac{1}{2} \nabla R+\frac{1}{2 \tau} X+2 \operatorname{Ric}(X, \cdot)=0 .
$$

This Euler-Lagrange equation is the analog to equation (4.2) above, the additional term $-\frac{1}{2} \nabla R$ comes from the additional $R$ in the definition of $\mathcal{L}(\gamma)$ and the term $2 \operatorname{Ric}(X, \cdot)$ comes from the evolution of $g$.

Let $C$ be a bound for both $|\mathrm{Ric}|$ and $|\nabla R|$ on $M \times\left[\tau_{0}, \bar{\tau}\right]$. For $\mathcal{L}$-geodesics, we find with 
$G(X)=0$ that

$$
\begin{aligned}
\partial_{\tau}|\sqrt{\tau} X|^{2} & =2\left\langle\partial_{\tau}(\sqrt{\tau} X), \sqrt{\tau} X\right\rangle+2 \operatorname{Ric}(\sqrt{\tau} X, \sqrt{\tau} X) \\
& =2 \tau\left\langle\frac{1}{2 \tau} X+\nabla_{X} X+\operatorname{Ric}(X, \cdot), X\right\rangle \\
& =2 \tau\left(\frac{1}{2}\langle\nabla R, X\rangle-\operatorname{Ric}(X, X)\right) \leq \tau C|X|+2 \tau C|X|^{2},
\end{aligned}
$$

and hence $\sqrt{\tau} X(\tau)$ has a limit as $\tau \rightarrow 0$ for $\mathcal{L}$-geodesics as in the static case. So we can extend the definition of $\mathcal{L}(\gamma)$ to $\tau_{0}=0$ in this case, which we will do from now on. We also fix $p \in M$. By $L(q, \bar{\tau})$ we denote the $\mathcal{L}$-length of an $\mathcal{L}$-shortest curve $\gamma(\tau)$, joining $p=\gamma(0)$ to $q=\gamma(\bar{\tau})$. Obviously, such a shortest curve is also an $\mathcal{L}$-geodesic.

We can then write the backwards reduced distance $\ell(q, \tau)$ from corollary 3.16 as

$$
\ell(q, \tau)=\frac{1}{2 \sqrt{\tau}} L(q, \tau) .
$$

We will now study the existence of minimizing $\mathcal{L}$-geodesics, and show some regularity results for $L(q, \tau)$ before continuing as in the static case above. Some of the following ideas can be found in [31].

We denote the initial standard distance between two points $p, q \in M$, i.e. the distance measured at time $\tau=0$ in the metric $g_{i j}(0)$, by $d(p, q)$. Because we assumed, that the curvatures of $(M, g(\tau))$ are uniformly bounded, we can find positive constants $k$ and $K$, such that $-k g \leq$ Ric $\leq K g$ for all $\tau \in[0, \bar{\tau}]$. We then get the following estimates on $\ell(q, \tau)$.

Lemma 4.5 (bounds on $\ell(q, \bar{\tau})$ )

The backwards reduced distance function satisfies the inequalities

$$
e^{-2 k \bar{\tau}} \frac{d^{2}(p, q)}{4 \bar{\tau}}-\frac{n k}{3} \bar{\tau} \leq \ell(q, \bar{\tau}) \leq e^{2 K \bar{\tau}} \frac{d^{2}(p, q)}{4 \bar{\tau}}+\frac{n K}{3} \bar{\tau} .
$$

Remark. Compared to the static case above, where $\ell(q, T)=d^{2}(p, q) / 4 T$, we have a multiplicative error term which comes from the evolution of the metric $g(\tau)$ and an additive error term which comes from the additional $R$ in the definition of the distance-functional $\mathcal{L}$. Lei $\mathrm{Ni}[27]$ used these bounds on $\ell$ to prove (3.25), analogous to our proof of (3.14) with $f \leq \ell=d^{2}(p, q) / 4 T$ for the static case.

Proof of lemma 4.5. The assumption $-k g \leq$ Ric implies $\partial_{\tau} g=2$ Ric $\geq-2 k g$ and hence we get

$$
\left.\log g\right|_{0} ^{\bar{\tau}}=\int_{0}^{\bar{\tau}} \frac{\partial_{\tau} g}{g} d \tau \geq \int_{0}^{\bar{\tau}}-2 k d \tau=-2 k \bar{\tau} .
$$

Exponentiating and multiplying by $g(0)$ yields

$$
g(\tau) \geq e^{-2 k \tau} g(0) .
$$

Recall that with the substitution $\lambda=\sqrt{\tau}$ above, we found

$$
\mathcal{L}(\gamma)=\int_{0}^{\sqrt{\bar{\tau}}}\left(2 \lambda^{2} R(\gamma(\lambda))+\frac{1}{2}\left|\gamma^{\prime}(\lambda)\right|^{2}\right) d \lambda .
$$


So for an arbitrary curve connecting $p$ to $q$, we find with (4.15)

$$
\mathcal{L}(\gamma) \geq \frac{e^{-2 k \bar{\tau}}}{2} \int_{0}^{\sqrt{\bar{\tau}}}\left|\gamma^{\prime}(\lambda)\right|_{g(0)}^{2} d \lambda-\frac{2 n k}{3} \bar{\tau}^{3 / 2} \geq e^{-2 k \bar{\tau}} \frac{d^{2}(p, q)}{2 \sqrt{\bar{\tau}}}-\frac{2 n k}{3} \bar{\tau}^{3 / 2} .
$$

With the definition $\ell(q, \bar{\tau})=\frac{1}{2 \sqrt{\bar{\tau}}} \inf _{\gamma \in \Gamma} \mathcal{L}(\gamma)$, we find the lower bound in the lemma. The upper bound can be proved in an analogous way.

Remark. This shows that the lower estimate $(4 \pi \bar{\tau})^{-n / 2} e^{-\ell(q, \bar{\tau})}$ of the adjoint heat kernel $u$ in corollary 3.16 converges to the same $\delta$-function as $u$ itself for $\tau \rightarrow 0$. So for small $\bar{\tau}$, our lower bound lies very close to the adjoint heat kernel $u$. Moreover, on the Euclidean space $\mathbb{R}^{n}$, we get with $k=K=0$ that $\ell(q, \bar{\tau})=\frac{d^{2}(p, q)}{4 \bar{\tau}}$ as in the static case, and hence $(4 \pi \bar{\tau})^{-n / 2} e^{-\ell(q, \bar{\tau})}$ is equal to the adjoint heat kernel for all $\bar{\tau} \geq 0$.

Definition 4.6 ( $\mathcal{L}$-exponential map at time $\bar{\tau}$ )

We define the $\mathcal{L}$-exponential map $\exp _{p}^{\mathcal{L}, \bar{\tau}}: T_{p} M \rightarrow M$ at the time $\bar{\tau}$ as follows. For $v \in T_{p} M$ let $\gamma_{v}$ be the $\mathcal{L}$-geodesic with $\gamma_{v}(0)=p$ and $\lim _{\tau \rightarrow 0} \sqrt{\tau} \dot{\gamma}(\tau)=v$. If $\gamma_{v}$ exists for $[0, \bar{\tau}]$, we set $\exp _{p}^{\mathcal{L}, \bar{\tau}}(v)=\gamma_{v}(\bar{\tau})$.

Remark. Note that unlike in the static case or in the classical Riemannian geometry case, the exponential map defined this way does not have to satisfy $\exp _{p}^{\mathcal{L}, \bar{\tau}}(0)=p$, because of the $\nabla R$ term in (4.13).

The $\mathcal{L}$-exponential map is defined on the whole tangent space $T_{p} M$. This can be shown similarly to the proof of the same result for the standard exponential map on a closed manifold. Moreover, there always exists a minimal $\mathcal{L}$-geodesic from $p$ to $q$, i.e. the $\mathcal{L}$ exponential map is onto. This can be seen as follows. For given points $p$ and $q$ in $M$, we let $\Gamma$ be the set of Sobolev curves $\gamma:[0, \tau] \rightarrow M$, joining $p=\gamma(0)$ to $q=\gamma(\tau)$. Then we take a sequence of curves $\gamma_{k} \in \Gamma$ with

$$
\lim _{k \rightarrow \infty} \mathcal{L}\left(\gamma_{k}\right)=\inf _{\gamma \in \Gamma} \mathcal{L}(\gamma)
$$

Because we found a lower bound for $\ell(q, \tau)$ which shows that $\ell(q, \tau) \rightarrow \infty$ as $d^{2}(p, q) \rightarrow \infty$, there is a weakly converging subsequence $\gamma_{k_{m}}$ and a Sobolev curve $\tilde{\gamma}$, such that $\gamma_{k_{m}} \rightarrow$ $\tilde{\gamma}(m \rightarrow \infty)$. Then $\mathcal{L}(\tilde{\gamma})=\inf _{\gamma \in \Gamma} \mathcal{L}(\gamma)$ and hence $\tilde{\gamma}$ must be an $\mathcal{L}$-geodesic, which proves the claim.

Our next concern will be the regularity of $\mathcal{L}$.

Definition 4.7 (injectivity domain)

We define the injectivity domain $\Omega(\tau)$ at time $\tau$ to be $\Omega(\tau):=\{q \in M \mid$ there is a unique minimal $\mathcal{L}$-geodesic $\gamma:[0, \tau] \rightarrow M$ with $\gamma(0)=p, \gamma(\tau)=q$ and $q$ is not conjugate to $p$ along $\gamma\}$. The condition of $q$ not being a conjugate point is understood as in the standard Riemannian geometry, namely there is no nontrivial $\mathcal{L}$-Jacobi field $J$ along $\gamma$ with $J(0)=$ $J(\tau)=0$. We also define the cut locus to be the set $C(\tau):=M \backslash \Omega(\tau)$.

Obviously $\ell(q, \tau)$ is smooth outside of $\bigcup_{\tau}(C(\tau) \times\{\tau\})$. Hence, if we can show that $\bigcup_{\tau}(C(\tau) \times$ $\{\tau\})$ is of measure zero, we have $\ell(q, \tau)$ smooth almost everywhere. With this regularity result, we can then assume that $\ell(q, \tau)$ is smooth on the whole space-time manifold $M \times[0, \bar{\tau}]$, and the inequalities we derive under this assumption will hold for general $\ell(q, \tau)$, too. 
Lemma 4.8 (Rugang Ye, [31])

The function $L(q, \tau)$ is locally Lipschitz continuous in both variables and the cut locus $C(\tau)$ is a closed set of measure zero for all $\tau$, hence $\bigcup_{\tau}(C(\tau) \times\{\tau\})$ is of measure zero, too.

Proof. We only explain the idea of the proof, the details can be found in [31]. We will not prove that $L(q, \tau)$ is locally Lipschitz. For the local Lipschitz continuity of $L(\cdot, \tau)$ with respect to the metric $g_{i j}(\tau)$ see [31], lemma 1.5. For the local Lipschitz continuity of $L(q, \cdot)$ for any fixed $q \in M$ see [31], lemma 1.6.

We set $C_{1}(\tau):=\{q \in M \mid \exists$ more than one minimal $\mathcal{L}$-geodesic from $p$ at time 0 to $q$ at time $\tau\}$, and $C_{2}(\tau):=\{q \in M \mid \exists$ a unique $\mathcal{L}$-geodesic $\gamma$ from $p=\gamma(0)$ to $q=\gamma(\tau)$ and $q$ is conjugate to $p$ along $\gamma\}$. Because $\exp _{p}^{\mathcal{L}, \tau}$ is onto, we get $C(\tau)=C_{1}(\tau) \cup C_{2}(\tau)$.

As in the standard Riemannian geometry, $C_{2}(\tau)$ is contained in the set of critical values of the $\mathcal{L}$-exponential map $\exp _{P}^{\mathcal{L}, \tau}$. Hence it has measure zero by Sard's theorem. At points in $C_{1}(\tau)$, the function $L(\cdot, \tau)$ is obviously not differentiable. But because it is Lipschitz, $L(\cdot, \tau)$ must be differentiable almost everywhere, so $C_{1}(\tau)$ is also a set of measure zero. Hence $C(\tau)$ has measure zero. Using the same arguments as in the standard Riemannian geometry, one can show that the injectivity domain is open, and hence $C(\tau)$ is closed. This proves the lemma.

Since $\ell(q, \tau)$ is smooth outside of the set $\bigcup_{\tau}(C(\tau) \times\{\tau\})$, i.e. smooth almost everywhere, we may always assume without loss of generality that $L(q, \tau)$ and $\ell(q, \tau)$ are $C^{\infty}$-functions on $M$. At the points in $\bigcup_{\tau}(C(\tau) \times\{\tau\})$, the inequalities which we compute in the following section will still hold in the barrier sense (and hence also in the sense of distributions).

\subsection{Monotonicity of the reduced volume}

Note that if Ric $=0$ on $M$, Perelman's $\mathcal{L}$-length is simply our $\mathcal{L}$-length from the static case above. So if Ric $=0$, the function $v(q, \tau):=(4 \pi \tau)^{-n / 2} e^{-\ell(q, \tau)}$ satisfies $\partial_{\tau} v-\triangle v=$ $-\partial_{t} v-\Delta v \leq 0$, i.e. $v$ is a subsolution to the backwards heat equation, which in the case $R=0$ is of course also the adjoint heat equation. This is in fact also true on a general manifold, namely we have the analogous to proposition 4.2.

Theorem 4.9 (Perelman, section 7 of [28])

Let $g_{i j}$ solve the backwards Ricci flow in $\tau=-t$. Let $\ell(q, \tau)$ be the backwards reduced distance defined above and set $v(q, \tau):=(4 \pi \tau)^{-n / 2} e^{-\ell(q, \tau)}$. Then $v(q, \tau)$ is a subsolution to the adjoint heat equation on $M$, i.e. $\square^{*} v=\partial_{\tau} v-\Delta v+R v \leq 0$.

Remark. Note that this theorem does not require any curvature assumptions converse to the static case!

We have divided the proof of theorem 4.9 into a sequence of lemmas.

Lemma 4.10 (equations for the backwards reduced distance)

Perelman's backwards reduced distance $\ell(q, \bar{\tau})$ satisfies the equations

$$
\begin{aligned}
|\nabla \ell|^{2} & =-R+\frac{1}{\bar{\tau}} \ell-\frac{1}{\bar{\tau}^{3 / 2}} K, \\
\partial_{\bar{\tau}} \ell & =-\frac{1}{\bar{\tau}} \ell+R+\frac{1}{2 \bar{\tau}^{3 / 2}} K,
\end{aligned}
$$


where

$$
K:=\int_{0}^{\bar{\tau}} \tau^{3 / 2} H(X) d \tau
$$

and $H(X)$ is Hamilton's trace Harnack inequality for the Ricci flow from theorem 2.15, evaluated at the negative time $t=-\tau$.

Proof. The following computations are analogous to the static case above. A minimizing curve satisfies (4.12) with $G(X)=0$. We hence get the first variation $\delta_{Y} L(q, \bar{\tau})=$ $2 \sqrt{\bar{\tau}}\langle X(\bar{\tau}), Y(\bar{\tau})\rangle=\langle\nabla L(q, \bar{\tau}), Y(\bar{\tau})\rangle$, and so the gradient of $L$ must be $\nabla L(q, \bar{\tau})=2 \sqrt{\bar{\tau}} X(\bar{\tau})$. This yields

$$
|\nabla L|^{2}=4 \bar{\tau}|X|^{2}=-4 \bar{\tau} R+4 \bar{\tau}\left(R+|X|^{2}\right) .
$$

Moreover, still analogous to the static case, we compute

$$
\begin{aligned}
\partial_{\bar{\tau}} L(q, \bar{\tau}) & =\frac{d}{d \bar{\tau}} L(q, \bar{\tau})-\nabla_{X} L(q, \bar{\tau}) \\
& =\sqrt{\bar{\tau}}\left(R+|X|^{2}\right)-\langle\nabla L(q, \bar{\tau}), X\rangle \\
& =\sqrt{\bar{\tau}}\left(R+|X|^{2}\right)-2 \sqrt{\bar{\tau}}|X|^{2} \\
& =2 \sqrt{\bar{\tau}} R-\sqrt{\bar{\tau}}\left(R+|X|^{2}\right) .
\end{aligned}
$$

Note again, that $\partial_{\bar{\tau}}$ denotes the partial derivative with respect to $\bar{\tau}$ keeping the point $q$ fixed, while $\frac{d}{d \bar{\tau}}$ refers to differentiation along an $\mathcal{L}$-geodesic, i.e. simultanously varying the time $\bar{\tau}$ and the point $q$. Next, we determine $\left(R+|X|^{2}\right)$ in terms of $L$. With the Euler-Lagrange equation (4.13), we get

$$
\begin{aligned}
\frac{d}{d \tau}\left(R(\gamma(\tau))+|X(\tau)|^{2}\right) & =\partial_{\tau} R+\nabla_{X} R+2\left\langle\nabla_{X} X, X\right\rangle+2 \operatorname{Ric}(X, X) \\
& =\partial_{\tau} R+2\langle\nabla R, X\rangle-\frac{1}{\tau}|X|^{2}-2 \operatorname{Ric}(X, X) \\
& =-H(X)-\frac{1}{\tau}\left(R+|X|^{2}\right),
\end{aligned}
$$

where

$$
\begin{aligned}
H(X) & =\partial_{t} R+\frac{1}{t} R-2\langle\nabla R, X\rangle+2 \operatorname{Ric}(X, X) \\
& =-\partial_{\tau} R-\frac{1}{\tau} R-2\langle\nabla R, X\rangle+2 \operatorname{Ric}(X, X)
\end{aligned}
$$

is exactly the expression from theorem 2.15 for Hamilton's trace Harnack inequality for the Ricci flow, evaluated at $t=-\tau$. So we get

$$
\tau^{3 / 2} \frac{d}{d \tau}\left(R+|X|^{2}\right)=-\tau^{3 / 2} H(X)-\sqrt{\tau}\left(R+|X|^{2}\right)
$$

and thus by integrating and using the notation $K=\int_{0}^{\bar{\tau}} \tau^{3 / 2} H(X) d \tau$, as in the lemma, one 
obtains

$$
\begin{aligned}
-K-L(q, \bar{\tau}) & =\int_{0}^{\bar{\tau}} \tau^{3 / 2} \frac{d}{d \tau}\left(R+|X|^{2}\right) d \tau \\
& =\bar{\tau}^{3 / 2}\left(R(\gamma(\bar{\tau}))+|X(\bar{\tau})|^{2}\right)-\int_{0}^{\bar{\tau}} \frac{3}{2} \sqrt{\tau}\left(R+|X|^{2}\right) d \tau \\
& =\bar{\tau}^{3 / 2}\left(R+|X|^{2}\right)-\frac{3}{2} L(q, \bar{\tau}) .
\end{aligned}
$$

Thus, we have

$$
\bar{\tau}^{3 / 2}\left(R+|X|^{2}\right)=-K+\frac{1}{2} L(q, \bar{\tau}) .
$$

If we insert this into (4.18) and (4.19) respectively, we get the equations for $L(q, \bar{\tau})$.

$$
\begin{aligned}
|\nabla L|^{2} & =-4 \bar{\tau} R+4 \bar{\tau}\left(R+|X|^{2}\right)=-4 \bar{\tau} R-\frac{4}{\sqrt{\bar{\tau}}} K+\frac{2}{\sqrt{\bar{\tau}}} L(q, \bar{\tau}), \\
\partial_{\bar{\tau}} L(q, \bar{\tau}) & =2 \sqrt{\bar{\tau}} R-\sqrt{\bar{\tau}}\left(R+|X|^{2}\right)=2 \sqrt{\bar{\tau}} R+\frac{1}{\bar{\tau}} K-\frac{1}{2 \bar{\tau}} L(q, \bar{\tau}) .
\end{aligned}
$$

Hence $\ell(q, \bar{\tau})=\frac{1}{2 \sqrt{\bar{\tau}}} L(q, \bar{\tau})$ satisfies

$$
|\nabla \ell|^{2}=-R+\frac{1}{2 \bar{\tau}^{3 / 2}} L-\frac{1}{\bar{\tau}^{3 / 2}} K=-R+\frac{1}{\bar{\tau}} \ell-\frac{1}{\bar{\tau}^{3 / 2}} K,
$$

which proves (4.16), as well as

$$
\partial_{\bar{\tau}} \ell=-\frac{1}{4 \bar{\tau}^{3 / 2}} L+R-\frac{1}{4 \bar{\tau}^{3 / 2}} L+\frac{1}{2 \bar{\tau}^{3 / 2}} K=-\frac{1}{\bar{\tau}} \ell+R+\frac{1}{2 \bar{\tau}^{3 / 2}} K,
$$

which proves (4.17).

To compute the second variation of $\mathcal{L}(\gamma)$, we need the following claim.

\section{Lemma 4.11}

Under backwards Ricci flow in $\tau$, we have

$$
\begin{aligned}
\partial_{\tau}\left\langle\nabla_{Y} Y, X\right\rangle= & \left\langle\nabla_{X} \nabla_{Y} Y, X\right\rangle+\left\langle\nabla_{Y} Y, \nabla_{X} X\right\rangle+2 \operatorname{Ric}\left(\nabla_{Y} Y, X\right) \\
& +2\left(\nabla_{Y} \operatorname{Ric}\right)(Y, X)-\left(\nabla_{X} \operatorname{Ric}\right)(Y, Y) .
\end{aligned}
$$

Proof. Apparently, we have

$$
\begin{aligned}
\partial_{\tau}\left\langle\nabla_{Y} Y, X\right\rangle= & \left\langle\nabla_{X} \nabla_{Y} Y, X\right\rangle+\left\langle\nabla_{Y} Y, \nabla_{X} X\right\rangle \\
& +2 \operatorname{Ric}\left(\nabla_{Y} Y, X\right)-\left\langle\dot{\nabla}_{Y} Y, X\right\rangle,
\end{aligned}
$$

where $\dot{\nabla}:=\partial_{t} \nabla=-\partial_{\tau} \nabla$. Proposition 1.10 says that

$$
\left\langle\dot{\nabla}_{U} V, W\right\rangle=-\left(\nabla_{U} \operatorname{Ric}\right)(V, W)+\left(\nabla_{W} \operatorname{Ric}\right)(U, V)-\left(\nabla_{V} \operatorname{Ric}\right)(U, W) .
$$

Hence, with $U=V=Y$ and $W=X$, we get

$$
\left\langle\dot{\nabla}_{Y} Y, X\right\rangle=-2\left(\nabla_{Y} \operatorname{Ric}\right)(Y, X)+\left(\nabla_{X} \operatorname{Ric}\right)(Y, Y)
$$

Inserting this into (4.22) proves the lemma. 
With equation (4.21), we can now write $2\left\langle\nabla_{Y} \nabla_{X} Y, X\right\rangle$ as

$$
\begin{aligned}
2\left\langle\nabla_{Y} \nabla_{X} Y, X\right\rangle= & 2\left\langle\nabla_{X} \nabla_{Y} Y, X\right\rangle+2\langle R(Y, X) Y, X\rangle \\
= & 2 \partial_{\tau}\left\langle\nabla_{Y} Y, X\right\rangle-2\left\langle\nabla_{Y} Y, \nabla_{X} X\right\rangle \\
& -4 \operatorname{Ric}\left(\nabla_{Y} Y, X\right)-4\left(\nabla_{Y} \operatorname{Ric}\right)(Y, X) \\
& +2\left(\nabla_{X} \operatorname{Ric}\right)(Y, Y)+2\langle R(Y, X) Y, X\rangle .
\end{aligned}
$$

A partial integration then yields

$$
\begin{aligned}
\int_{0}^{\bar{\tau}} 2 \sqrt{\tau}\left\langle\nabla_{Y} \nabla_{X} Y, X\right\rangle d \tau= & \left.2 \sqrt{\tau}\left\langle\nabla_{Y} Y, X\right\rangle\right|_{0} ^{\bar{\tau}}-\int_{0}^{\bar{\tau}} \sqrt{\tau} \frac{1}{\tau}\left\langle\nabla_{Y} Y, X\right\rangle d \tau \\
& -\int_{0}^{\bar{\tau}} 2 \sqrt{\tau}\left\langle\nabla_{Y} Y, \nabla_{X} X+2 \operatorname{Ric}(X, \cdot)\right\rangle d \tau \\
& -\int_{0}^{\bar{\tau}} \sqrt{\tau}\left(4\left(\nabla_{Y} \operatorname{Ric}\right)(Y, X)-2\left(\nabla_{X} \operatorname{Ric}\right)(Y, Y)\right) d \tau \\
& +\int_{0}^{\bar{\tau}} 2 \sqrt{\tau}\langle R(Y, X) Y, X\rangle d \tau
\end{aligned}
$$

If the geodesic equation $G(X)=\nabla_{X} X-\frac{1}{2} \nabla R+\frac{1}{2 \tau} X+2 \operatorname{Ric}(X, \cdot)=0$ holds, we can write the first two integrals on the left hand side of (4.23) as

$$
-2 \int_{0}^{\bar{\tau}} \sqrt{\tau}\left\langle\nabla_{Y} Y, \frac{1}{2 \tau} X+\nabla_{X} X+2 \operatorname{Ric}(X, \cdot)\right\rangle d \tau=-\int_{0}^{\bar{\tau}} \sqrt{\tau}\left\langle\nabla_{Y} Y, \nabla R\right\rangle d \tau
$$

and so equation (4.23) becomes

$$
\begin{aligned}
\int_{0}^{\bar{\tau}} 2 \sqrt{\tau}\left\langle\nabla_{Y} \nabla_{X} Y, X\right\rangle d \tau= & \left.2 \sqrt{\tau}\left\langle\nabla_{Y} Y, X\right\rangle\right|_{0} ^{\bar{\tau}}-\int_{0}^{\bar{\tau}} \sqrt{\tau}\left\langle\nabla_{Y} Y, \nabla R\right\rangle d \tau \\
& -\int_{0}^{\bar{\tau}} \sqrt{\tau}\left(4\left(\nabla_{Y} \operatorname{Ric}\right)(Y, X)-2\left(\nabla_{X} \operatorname{Ric}\right)(Y, Y)\right) d \tau \\
& +\int_{0}^{\bar{\tau}} 2 \sqrt{\tau}\langle R(Y, X) Y, X\rangle d \tau
\end{aligned}
$$

We can now compute the second variation of $\mathcal{L}(\gamma)$ for $\mathcal{L}$-geodesics $\gamma$, where $G(X)=0$ is satisfied. Using the first variation

$$
\delta_{Y} \mathcal{L}(\gamma)=\int_{\tau_{0}}^{\bar{\tau}} \sqrt{\tau}\left(\nabla_{Y} R+2\left\langle\nabla_{Y} X, X\right\rangle\right) d \tau
$$


from (4.11), we compute

$$
\begin{aligned}
\delta_{Y}^{2} \mathcal{L}(\gamma)= & \int_{0}^{\bar{\tau}} \sqrt{\tau}\left(\partial_{s}\langle\nabla R, Y\rangle+2\left\langle\nabla_{Y} \nabla_{Y} X, X\right\rangle+2\left|\nabla_{Y} X\right|^{2}\right) d \tau \\
= & \int_{0}^{\bar{\tau}} \sqrt{\tau}\left(\left\langle\nabla R, \nabla_{Y} Y\right\rangle+\nabla_{Y} \nabla_{Y} R+2\left|\nabla_{X} Y\right|^{2}\right) d \tau \\
& +\int_{0}^{\bar{\tau}} 2\left\langle\nabla_{Y} \nabla_{X} Y, X\right\rangle d \tau \\
= & \left.2 \sqrt{\tau}\left\langle\nabla_{Y} Y, X\right\rangle\right|_{0} ^{\bar{\tau}}+\int_{0}^{\bar{\tau}} \sqrt{\tau}\left(\nabla_{Y} \nabla_{Y} R+2\left|\nabla_{X} Y\right|^{2}\right) d \tau \\
& +\int_{0}^{\bar{\tau}} \sqrt{\tau}\left(2\left(\nabla_{X} \operatorname{Ric}\right)(Y, Y)-4\left(\nabla_{Y} \operatorname{Ric}\right)(Y, X)\right) d \tau \\
& +\int_{0}^{\bar{\tau}} 2 \sqrt{\tau}\langle R(Y, X) Y, X\rangle d \tau
\end{aligned}
$$

where we used (4.24) in the last step. As in the static case, we now consider a special test variation to estimate the Hessian of $L$. Namely, we take a normalized vector $Y(\bar{\tau})$ in $T_{q} M$ and construct $Y(\tau)$ on $[0, \bar{\tau}]$ as the solution of the ODE

$$
\nabla_{X} Y=-\operatorname{Ric}(Y, \cdot)+\frac{1}{2 \tau} Y
$$

As above, we find $\partial_{\tau}|Y|^{2}=2 \operatorname{Ric}(Y, Y)+2\left\langle\nabla_{X} Y, Y\right\rangle=\frac{1}{\tau}|Y|^{2}$ and hence $|Y(\tau)|^{2}=\tau / \bar{\tau}$, in particular $Y(0)=0$. We have

$$
\begin{aligned}
\operatorname{Hess}_{L}(Y, Y) & =\nabla_{Y} \nabla_{Y} L=\delta_{Y}^{2}(L)-\left\langle\nabla_{Y} Y, \nabla L\right\rangle \\
& \leq \delta_{Y}^{2} \mathcal{L}-2 \sqrt{\bar{\tau}}\left\langle\nabla_{Y} Y, X\right\rangle(\bar{\tau}),
\end{aligned}
$$

where the $Y$ in $\operatorname{Hess}_{L}(Y, Y)=\nabla_{Y} \nabla_{Y} L$ denotes a vector $Y(\bar{\tau}) \in T_{q} M$, while in $\delta_{Y}^{2}(L)$ it denotes the associated variation of the curve, i.e. the vector field $Y(\tau)$ along $\gamma$ which solves the above ODE (4.26). Note that the inequality becomes an equality if $Y$ is an $\mathcal{L}$-Jacobi field, which are exactly the minimizers of the expression on the second line. This is explained in detail in [23]. Inserting the formula (4.25) for the second variation, we find

$$
\begin{aligned}
\operatorname{Hess}_{L}(Y, Y) \leq & \int_{0}^{\bar{\tau}} \sqrt{\tau}\left(\nabla_{Y} \nabla_{Y} R+2\left|\nabla_{X} Y\right|^{2}+2\langle R(Y, X) Y, X\rangle\right) d \tau \\
& +\int_{0}^{\bar{\tau}} \sqrt{\tau}\left(2\left(\nabla_{X} \operatorname{Ric}\right)(Y, Y)-4\left(\nabla_{Y} \operatorname{Ric}\right)(Y, X)\right) d \tau .
\end{aligned}
$$

We can now prove an estimate for the Laplacian of $\ell(q, \bar{\tau})$ as in the static case.

\section{Lemma 4.12}

For $K$ defined as in lemma 4.10, the backwards reduced distance $\ell(q, \bar{\tau})$ satisfies

$$
\triangle \ell(q, \bar{\tau}) \leq \frac{n}{2 \bar{\tau}}-R-\frac{1}{2 \bar{\tau}^{3 / 2}} K .
$$

Proof. Note that with (4.26) we find

$$
\begin{aligned}
\left|\nabla_{X} Y\right|^{2} & =|\operatorname{Ric}(Y, \cdot)|^{2}-\frac{1}{\tau} \operatorname{Ric}(Y, Y)+\frac{1}{4 \tau^{2}}|Y(\tau)|^{2} \\
& =|\operatorname{Ric}(Y, \cdot)|^{2}-\frac{1}{\tau} \operatorname{Ric}(Y, Y)+\frac{1}{4 \tau \bar{\tau}} .
\end{aligned}
$$


Moreover, (4.26) also implies

$$
\begin{aligned}
\frac{d}{d \tau} \operatorname{Ric}(Y(\tau), Y(\tau))= & \left(\partial_{\tau} \operatorname{Ric}\right)(Y, Y)+\left(\nabla_{X} \operatorname{Ric}\right)(Y, Y)+2 \operatorname{Ric}\left(\nabla_{X} Y, Y\right) \\
= & \left(\partial_{\tau} \operatorname{Ric}\right)(Y, Y)+\left(\nabla_{X} \operatorname{Ric}\right)(Y, Y) \\
& +\frac{1}{\tau} \operatorname{Ric}(Y, Y)-2|\operatorname{Ric}(Y, \cdot)|^{2} .
\end{aligned}
$$

By plugging equation (4.29) into (4.27), we obtain

$$
\begin{aligned}
\operatorname{Hess}_{L}(Y, Y) \leq & \int_{0}^{\bar{\tau}} \sqrt{\tau}\left(\nabla_{Y} \nabla_{Y} R+2\langle R(Y, X) Y, X\rangle\right) d \tau \\
& +\int_{0}^{\bar{\tau}} \sqrt{\tau}\left(2\left(\nabla_{X} \operatorname{Ric}\right)(Y, Y)-4\left(\nabla_{Y} \operatorname{Ric}\right)(Y, X)\right) d \tau . \\
& +\int_{0}^{\bar{\tau}} \sqrt{\tau}\left(2|\operatorname{Ric}(Y, \cdot)|^{2}-\frac{2}{\tau} \operatorname{Ric}(Y, Y)+\frac{1}{2 \tau \bar{\tau}}\right) d \tau
\end{aligned}
$$

and with a partial integration and using (4.30), we find

$$
\begin{aligned}
\operatorname{Hess}_{L}(Y, Y) \leq & \frac{1}{\sqrt{\bar{\tau}}}-\int_{0}^{\bar{\tau}} \sqrt{\tau} H(X, Y) d \tau \\
& -\int_{0}^{\bar{\tau}} \sqrt{\tau}\left(2\left(\nabla_{X} \operatorname{Ric}\right)(Y, Y)-4|\operatorname{Ric}(Y, \cdot)|^{2}\right) d \tau \\
& -\int_{0}^{\bar{\tau}} \sqrt{\tau}\left(\frac{3}{\tau} \operatorname{Ric}(Y, Y)+2\left(\partial_{\tau} \operatorname{Ric}\right)(Y, Y)\right) d \tau \\
= & \frac{1}{\sqrt{\bar{\tau}}}-\int_{0}^{\bar{\tau}} \sqrt{\tau} H(X, Y) d \tau \\
& -\int_{0}^{\bar{\tau}} \sqrt{\tau}\left(2 \frac{d}{d \tau} \operatorname{Ric}(Y, Y)+\frac{1}{\tau} \operatorname{Ric}(Y, Y)\right) d \tau \\
= & \frac{1}{\sqrt{\bar{\tau}}}-2 \sqrt{\bar{\tau}} \operatorname{Ric}(Y, Y)-\int_{0}^{\bar{\tau}} \sqrt{\tau} H(X, Y) d \tau,
\end{aligned}
$$

where

$$
\begin{aligned}
H(X, Y)= & 2\left(\partial_{t} \operatorname{Ric}\right)(Y, Y)+2|\operatorname{Ric}(Y, \cdot)|^{2}-\nabla_{Y} \nabla_{Y} R+\frac{1}{t} \operatorname{Ric}(Y, Y) \\
& -4\left[\left(\nabla_{X} \operatorname{Ric}\right)(Y, Y)-\left(\nabla_{Y} \operatorname{Ric}\right)(X, Y)\right]-2\langle R(X, Y) X, Y\rangle \\
= & -2\left(\partial_{\tau} \operatorname{Ric}\right)(Y, Y)+2|\operatorname{Ric}(Y, \cdot)|^{2}-\nabla_{Y} \nabla_{Y} R-\frac{1}{\tau} \operatorname{Ric}(Y, Y) \\
& -4\left[\left(\nabla_{X} \operatorname{Ric}\right)(Y, Y)-\left(\nabla_{Y} \operatorname{Ric}\right)(X, Y)\right]-2\langle R(X, Y) X, Y\rangle
\end{aligned}
$$

is exactly Hamilton's matrix Harnack expression for the Ricci flow evaluated at the negative time $t=-\tau$, see theorem 2.15 .

We now let $\left\{Y_{i}\right\}$ be an orthonormal basis of $T_{q} M$, and define $Y_{i}(\tau)$ as above, as the solutions of the ODE (4.26). Then there exist vector fields $\tilde{Y}_{i}(\tau)$ along $\gamma$ with $|\tilde{Y}(\tau)| \equiv 1$ and 
$Y_{i}(\tau)=\sqrt{\tau / \bar{\tau}} \tilde{Y}_{i}(\tau)$. Summing (4.31) over all $i=1, \ldots, n$ yields

$$
\begin{aligned}
\triangle L(q, \bar{\tau}) & \leq \sum_{i=1}^{n}\left(\frac{1}{\sqrt{\bar{\tau}}}-2 \sqrt{\bar{\tau}} \operatorname{Ric}\left(Y_{i}, Y_{i}\right)-\int_{0}^{\bar{\tau}} \sqrt{\tau} H\left(X, Y_{i}\right) d \tau\right) \\
& =\frac{n}{\sqrt{\bar{\tau}}}-2 \sqrt{\bar{\tau}} R-\frac{1}{\bar{\tau}} \int_{0}^{\bar{\tau}} \tau^{3 / 2} \sum_{i=1}^{n} H\left(X, \tilde{Y}_{i}\right) d \tau \\
& =\frac{n}{\sqrt{\bar{\tau}}}-2 \sqrt{\bar{\tau}} R-\frac{1}{\bar{\tau}} \int_{0}^{\bar{\tau}} \tau^{3 / 2} H(X) d \tau \\
& =\frac{n}{\sqrt{\bar{\tau}}}-2 \sqrt{\bar{\tau}} R-\frac{1}{\bar{\tau}} K,
\end{aligned}
$$

where $K=\int_{0}^{\bar{\tau}} \tau^{3 / 2} H(X) d \tau$ as above. Here we used $\sum_{i=1}^{n} H\left(X, \tilde{Y}_{i}\right)=H(X)$, which we proved in chapter two. Finally, for $\ell(q, \bar{\tau})$ this yields

$$
\triangle \ell(q, \bar{\tau})=\frac{1}{2 \sqrt{\bar{\tau}}} \triangle L(q, \bar{\tau}) \leq \frac{n}{2 \bar{\tau}}-R-\frac{1}{2 \bar{\tau}^{3 / 2}} K .
$$

This finishes the proof of the lemma.

We can now prove our main theorem.

Proof of theorem 4.9. We need the three equations (4.16), (4.17) and (4.28) from lemma 4.10 and lemma 4.12, i.e.

$$
\begin{aligned}
|\nabla \ell|^{2} & =-R+\frac{1}{\bar{\tau}} \ell-\frac{1}{\bar{\tau}^{3 / 2}} K, \\
\partial_{\bar{\tau}} \ell & =-\frac{1}{\bar{\tau}} \ell+R+\frac{1}{2 \bar{\tau}^{3 / 2}} K, \\
\triangle \ell & \leq \frac{n}{2 \bar{\tau}}-R-\frac{1}{2 \bar{\tau}^{3 / 2}} K .
\end{aligned}
$$

This yields

$$
\partial_{\bar{\tau}} \ell-\triangle \ell+|\nabla \ell|^{2}-R+\frac{n}{2 \bar{\tau}} \geq 0,
$$

which is apparently equivalent to $\square^{*}\left[(4 \pi \bar{\tau})^{-n / 2} e^{\ell(-q, \bar{\tau})}\right] \leq 0$, compare with (3.21). This proves the theorem.

Remark. In the static manifold case, we had

$$
\triangle \ell(q, T) \leq \frac{n}{2 T}-\frac{1}{2 T^{3 / 2}} \int_{0}^{T} 2 t^{3 / 2} \operatorname{Ric}(X, X) d t
$$

and needed to assume that Ric $\geq 0$ to get the result we wanted. But here, in

$$
\triangle \ell(q, \bar{\tau}) \leq \frac{n}{2 \bar{\tau}}-R-\frac{1}{2 \bar{\tau}^{3 / 2}} \int_{0}^{\bar{\tau}} \tau^{3 / 2} H(X) d \tau,
$$

we do not need to assume that $H(X) \geq 0$, which one could guess by analogy. The $K$-terms cancel out, even if the Harnack inequality $H(X) \geq 0$ does not hold! We have seen similar results before, where we needed Ric $\geq 0$ in the static case, whereas under the Ricci flow everything just works fine without additional assumptions. Thus, even so the computations are longer and more difficult in the Ricci flow case, the results are more natural and hold without a-priori assumptions on the curvature. 
Corollary 4.13 (monotonicity of backwards reduced volume)

The backwards reduced volume

$$
\tilde{V}(\tau):=\int_{M}(4 \pi \tau)^{-n / 2} e^{-\ell(q, \tau)} d V(q)
$$

is non-increasing in $\tau$ under the backwards Ricci flow in $\tau$.

Proof. With theorem 4.9, $v(q, \tau)$ defined by $v(q, \tau):=(4 \pi \tau)^{-n / 2} e^{-\ell(q, \tau)}$ and with $\square^{*}=$ $\partial_{\tau}-\triangle+R$, we get

$$
\begin{aligned}
\partial_{\tau} \tilde{V}(\tau) & =\int_{M} \partial_{\tau} v d V+\int_{M} v \partial_{\tau} d V \\
& \leq \int_{M}(\triangle-R) v d V+\int_{M} R v d V \\
& =\int_{M} \triangle v d V=0 .
\end{aligned}
$$

This proves the corollary.

In corollary 3.16 we have seen, that $(4 \pi \tau)^{-n / 2} e^{-\ell(q, \tau)}$ is a lower bound on the adjoint heat kernel $(4 \pi \tau)^{-n / 2} e^{-f(q, \tau)}$. In particular, we have $\ell(q, \tau) \geq f(q, \tau)$. With the estimates from corollary 4.5 we have seen above, that $\ell(q, \tau)$ converges to a $\delta$-function as $\tau \rightarrow 0$ and hence $\ell(q, \tau) \rightarrow f(q, \tau)$ as $\tau \rightarrow 0$. Now the backwards reduced volume above is constant if and only if $\ell(q, \tau)$ satisfies the adjoint heat equation (instead on being a subsolution). But this immediately implies that $\ell(q, \tau)=f(q, \tau)$ for all $\tau \geq 0$, and hence we are on a gradient shrinking soliton with potential $f=\ell$. This shows that the backwards reduced volume is in fact strictly decreasing in $\tau$, unless we are on a gradient shrinking soliton.

Remark. All the above computations can also be made for the forward reduced volume, i.e. for the expanding case instead of the shrinking case. Again, one only has to change some signs. In this case one does not have to evaluate Hamilton's Harnack expressions at negative times, but for positive $t$, so the interpretations actually sometimes slightly differ. However, the result at the end is analogous, namely we get for the forward reduced distance $\ell(q, t)$ that $(4 \pi t)^{-n / 2} e^{-\ell(q, t)}$ is a supersolution to the adjoint heat equation. Moreover, the forward reduced volume is monotone non-increasing in $t$ along the Ricci flow and constant precisely on gradient expanders.

A remarkable fact is, that the shrinker-entropy $\mathcal{W}$ and the expander-entropy $\mathcal{W}_{+}$both increase in forward time, while the reduced volumes both increase as $t$ approaches the reference time $t=0$.

Again, the details for the expanding case can be found in [12].

\section{References}

[1] S.B. Angenent and D. Knopf, An Example Of neckpinching for Ricci Flow on $S^{n+1}$, Mathematical Research Letters 11 (2004), no. 4, 493-518.

[2] S.B. Angenent and D. Knopf, Precise asymptotics for the Ricci flow neckpinch, arXiv:math.DG/0511247 (2005).

[3] H.-D. Cao and B. Chow, Recent Developments on the Ricci Flow, Bulletin of the American Mathematical Society 36 (1999) 59-74. 
[4] H.-D. Cao and X.-P. Zhu, A Complete Proof of the Poincaré and Geometrization Conjectures - application of the Hamilton-Perelman theory of the Ricci flow, Asian Journal of Mathematics 10 (2006), no. 2, 165-492.

[5] B. Chow and S.-C. Chu, A Geometric Interpretation of Hamilton's Harnack Inequality for the Ricci Flow, Mathematical Research Letters 2 (1995) 701-718.

[6] B. Chow and S.-C. Chu, A Geometric Approach to the Linear Trace Harnack Inequality for the Ricci Flow, Mathematical Research Letters 3 (1996) 549-568.

[7] H.-D. Cao, R.S. Hamilton and T. Ilmanen, Gaussian Densities and Stability for some Ricci Solitons, arXiv:math.DG/0404165 (2004).

[8] B. Chow and D. Knopf, The Ricci Flow: An Introduction, American Mathematical Society, 2004.

[9] D. DeTurck, Deforming Metrics in the Direction of their Ricci Tensor, Journal of Differential Geometry 18 (1983) 157-162.

[10] J. Eells and J.H. Sampson, Harmonic Mappings of Riemannian Manifolds, American Journal of Mathematics 86 (1964) 109-160.

[11] L.C. Evans, Partial Differential Equations, American Mathematical Society, 1998.

[12] M. Feldman, T. Ilmanen and L. Ni, Entropy and Reduced Distance for Ricci Expanders, arXiv:math.DG/0405036 (2004).

[13] R.S. Hamilton, Three-Manifolds with Positive Ricci Curvature, Journal of Differential Geometry 17 (1982) 255-306.

[14] R.S. Hamilton, Four-manifolds with Positive Curvature Operator, Journal of Differential Geometry 24 (1986) 153-179.

[15] R.S. Hamilton, Four-manifolds with Positive Isotropic Curvature, Communications in Analytic Geometry 5 (1997) 1-92.

[16] R.S. Hamilton, The Ricci Flow on Surfaces, Contemporary Mathematics 71 (1988) 237-267.

[17] R.S. Hamilton, A Matrix Harnack Estimate for the Heat Equation, Communications in Analysis and Geometry 1 (1993) 113-126.

[18] R.S. Hamilton, The Harnack Estimate for the Ricci Flow, Journal of Differential Geometry 41 (1995) 215-226.

[19] R.S. Hamilton, The Formation of Singularities in the Ricci Flow, Surveys in Differential Geometry 2 (1995) 7-136.

[20] T. Ilmanen, Lecture Notes on the Ricci Flow, Lecture at ETH Zürich, Summer semester 2004, unpublished.

[21] J. Jost, Riemannian Geometry and Geometric Analysis, Third Edition, Springer 2002.

[22] P. Li and S.-T. Yau, On the Parabolic Kernel of the Schrödinger Operator, Acta Mathematica 156 (1986) 153-201.

[23] B. Kleiner, J. Lott, Notes on Perelman's Papers (May 2006 version), arXiv:math.DG/0605667 (2006).

[24] J. Moser, A Harnack Inequality for Parabolic Differential Equations, Communications on Pure and Applied Mathematics 17 (1964) 101-134.

[25] L. Ni, The Entropy Formula for Linear Heat Equation, Journal of Geometric Analysis 14,1 (2004) 85-98.

[26] L. Ni, Addenda to "The Entropy Formula for Linear Heat Equation", Journal of Geometric Analysis 14,2 (2004) 329-334.

[27] L. Ni, A Note on Perelman's LYH Inequality, arXiv:math.DG/0602337 (2006).

[28] G. Perelman, The Entropy Formula for the Ricci Flow and its Geometric Applications, arXiv:math.DG/0211159 (2002).

[29] W. Thurston, Three-dimensional Manifolds, Kleinian Groups and Hyperbolic Geometry, Bulletin of the American Mathematical Society 6 (1982) 357-381.

[30] P. Topping, Lectures on the Ricci Flow, http://www.maths.warwick.ac.uk/ topping/RFnotes.html (2005). 
[31] R. Ye, Notes on the Reduced Volume and Asymptotic Ricci Solitons of $\kappa$-Solutions, http://www.math.ucsb.edu/ yer/ricciflow.html (2004).

Reto Müller

ETH ZÜRICH, 8092 ZÜRICH, SwITZERLAND 